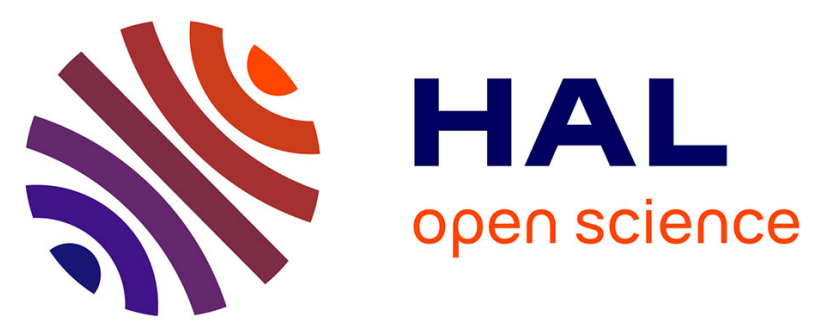

\title{
Les styles céramiques du Néolithique moyen en Languedoc oriental : caractérisation et premières comparaisons avec la périodisation des industries lithiques taillées \\ Catherine Georjon, Vanessa Léa
}

\section{To cite this version:}

Catherine Georjon, Vanessa Léa. Les styles céramiques du Néolithique moyen en Languedoc oriental: caractérisation et premières comparaisons avec la périodisation des industries lithiques taillées. Gallia Préhistoire - Préhistoire de la France dans son contexte européen, 2013, 55, pp.31-71. 10.3406/galip.2013.2499 . hal-01956992v2

\section{HAL Id: hal-01956992 \\ https://hal.science/hal-01956992v2}

Submitted on 19 Dec 2019

HAL is a multi-disciplinary open access archive for the deposit and dissemination of scientific research documents, whether they are published or not. The documents may come from teaching and research institutions in France or abroad, or from public or private research centers.
L'archive ouverte pluridisciplinaire HAL, est destinée au dépôt et à la diffusion de documents scientifiques de niveau recherche, publiés ou non, émanant des établissements d'enseignement et de recherche français ou étrangers, des laboratoires publics ou privés.

\section{(1) (1) $\$$}

Distributed under a Creative Commons Attribution - NonCommercial - NoDerivatives 44.0 


\title{
Les styles céramiques du Néolithique moyen en Languedoc oriental : caractérisation et premières comparaisons avec la périodisation des industries lithiques taillées
}

\author{
Cathy GeORJON* \& Vanessa LÉA ${ }^{* *}$
}

Mots CLÉs. Néolithique moyen I, Néolithique moyen II, Chasséen, céramique, industries lithiques taillées

RÉSUMÉ. À la suite de l'analyse croisée des composantes céramiques de 12 séries du Languedoc Oriental et de trois séries de comparaison extra-régionales, 5 groupes se dégagent, nommés styles chasséens 1 à 5, calées chronologiquement par des datations radiocarbones encore peu nombreuses. Le style 1 peut être considéré comme un Proto-Chasséen, c'est-à-dire comme un Chasséen incomplet ; il se situerait entre 4340 et 4250 av. J.-C. Le style 2 se compose de deux variantes, et se rapproche du style des Plots. II se situe entre 4310 et 3985 av. J.-C. Le style 3 présente également deux variantes. La variante 3 a est une combinaison d'éléments archaïques en voie de raréfaction et de formes nouvelles et variées, datées entre 3965 et 3805 av. J.-C. La variante 3b s'est affranchie de ces archaïsmes; elle n'est pas datée. Le style 4 correspond au style d'Auriac. Enfin, le style 5 se démarque nettement des autres. Deux variantes sont également possibles. Parmi les formes emblématiques de ce style on trouve les fameuses "écuelles de Bize ». Pour ces deux derniers styles, le Radiocarbone donne un intervalle de 3820 à 3650 av. J.-C. Antérieurement au Chasséen, on distingue quelques ensembles restreints au sein desquels se marque nettement l'ascendance du Néolithique ancien ; ils sont datés entre 4555 et 4460 av. J.-C. La fin de la séquence du Néolithique moyen est représentée par un site qui se démarque des productions chasséennes tout en demeurant dans le giron des groupes à céramique lisse ; il est daté entre 3640 et 3520 av. J.-C. Les industries lithiques taillées du Chasséen évoluent selon un rythme plus lent dont les moments de transition ne correspondent pas toujours à des césures dans les styles céramiques. Trois styles lithiques sont identifiables (A, B et C). La confrontation des périodisations du Midi fait ressortir une complexification croissante où les processus d'évolution et de régionalisation semblent s'accélérer dans les phases récentes du Chasséen. $\begin{array}{lrrrr}\text { Key Words. } & \text { Middle } & \text { Neolithic I, } & \text { Middle } & \text { Neolithic II, } \\ \text { Chassey } & \text { culture, } & \text { pottery, } & \text { lithic } & \text { industry }\end{array}$

ABSTRACT. Further to the cross-analysis of the ceramic components of 12 series of Eastern Languedoc and 3 extra-regional comparison series, 5 groups emerge named chassean style 1 to 5 . They are chronologically situated by still few radiocarbon dates. Style 1 can be considered like a Proto-Chassean, is to say as an incomplete Chassey culture would be between 4340 and 4250 cal BC. Style 2 consists of two variants, and is closer to the style of Les Plots. It is dated between 4310 and 3985 cal BC. Style 3 also consists of two variants. Scarcest archaic elements and varied new ceramic forms make up the variant 3a dated between 3965 and $3805 \mathrm{cal} \mathrm{BC}$. The style $3 \mathrm{~b}$, which is undated, emerges from these archaisms. Style 4 which is closer to the style of Auriac and style 5 that includes the "écuelles de Bize" can't be distinguished by radiocarbon dating. They are between 3820 and 3650 cal BC. Before Chassey culture, there are a few small sets with early Neolithic features dated between 4555 and $4460 \mathrm{cal}$ BC. The end of the middle Neolithic sequence is represented by a set free of the chassean influence dated between 3640 and 3520 cal BC. The chassean lithic industries move at a slower pace whose changes do not always meet those of ceramic styles. Three lithic styles are identified (style A, B and C). Confronting the periodisations of Southern Chassey culture shows an increasingly complex nature of evolutionary process especially during the recent phases.

Translation: Louise Byrne

\footnotetext{
* INRAP, laboratoire TRACES - UMR5608, Université de Toulouse 2 le Mirail, Maison de la Recherche Bât. 26, 5, allée Antonio MACHADO, 31058 Toulouse Cedex 9.

** CNRS, laboratoire TRACES - UMR5608, Université de Toulouse 2 le Mirail, Maison de la Recherche Bât. 26, 5, allée Antonio MACHADO, 31058 Toulouse Cedex 9.
} 


\section{Middle Neolithic ceramic styles in eastern Languedoc: characterization and first comparisons with the periodization of chipped lithic industries}

During the past ten years, $\mathrm{PhD}$ research results concerning the Garonne (François, 2002; 2007; Gandelin, 2011), Quercy (Gernigon, 2004) and Provence regions (Lepère 2009; 2012), have increased our knowledge of the seriation of southern Chassean ceramic data. Such research represents significant progress compared with that conducted in the 1980s-1990s, as it develops the methodical, critical and exhaustive analysis of extensive corpora.

In this context, eastern Languedoc represents the missing link in the southern Chassean seriation of ceramic series as, up until now, it has only benefited from an article of limited scope (Georjon, 2003). It is all the more important to propose more advanced work for Chassean pottery items from eastern Languedoc given that the associated lithic industries have been the subject of a research thesis (Léa, 2004a).

This article therefore proposes the periodization of pottery data for eastern Languedoc based on automatic seriation and factorial correspondence analysis. The groups obtained as a result of these analyses are placed in the context of existing southern Chassean periodization. This study is combined with a review of the available radiocarbon chronology and an attempt to harmonise the periodization of pottery and chipped lithic industries. Finally, a more general discussion is developed concerning the position of eastern Languedoc in southern Chassean culture.

Eastern Languedoc is located between the Rhône, Ardèche and Hérault rivers. It is composed of three natural stepped entities which run along a southwest-northeast axis, which are, from the nearest to the furthest from the sea: the coastal plain, foothill garrigues and the Causses slopes. Most of the Neolithic sites are located in the coastal plain (fig. 1).

The corpus of ceramic series analysed here, most of which are unpublished, comes from twelve sites in eastern Languedoc. For comparative purposes, we associated this corpus with three ceramic series from two sites in Provence and one in western Languedoc (fig. 2). The corpus of variables is composed of 31 elements (fig. 3), made up of pottery from reconstituted recipients and fragmentary typology elements. The processing of ceramic data is carried out with automatic seriation (fig. 4) and factorial correspondence analyses in association with cluster analyses (fig. 5 to 9). Following these cross-analyses, 5 groups emerge, named Chassean styles 1 to 5 (fig. 10 to 22). These are chronologically classified by means of the few available radiocarbon dates (fig. 24 and 25).

Style 1 (fig. 10 and 11) can be considered as ProtoChassean, that is to say, as an incomplete Chassean, between 4340 and 4250 cal BC. Style 2 (fig. 12 to 14) consists of two variants; $2 \mathrm{a}$ and $2 \mathrm{~b}$. Style $2 \mathrm{a}$ is essentially composed of sets in which numerous carinated containers are associated with globular or sinuous shapes. In style $2 \mathrm{~b}$, carinated forms are rare, simple plates and bowls with internal beading are well represented, as are jars encircled by a band; elements that define the style of Les Plots (Vaquer, 1990; 1991). Styles 2a and $2 \mathrm{~b}$ are dated between 4310 and 3985 cal BC. Style 3 (fig. 15 to 17) also consists of two variants. Rarer archaic elements and varied new ceramic forms make up variant $3 \mathrm{a}$, dated between 3965 and 3805 cal BC. Style 3b, which is undated, no longer includes these archaic features and the diversity of segmented forms is on the rise. Style 4 (fig. 18 and 19) is characterised by carinated plates, like those in the style of Auriac (Vaquer, 1990; 1991; Jédikian, 2000) and by the development of internal decoration on simple bowls and plates. Lastly, style 5 (fig. 20 to 22) is the one that stands out the most from the others. There are also two potential variants. Among the emblematic forms of this style are the famous "bowls of Bize" (Vaquer, 1990; 1991; Guilaine, 1997b). The two latter styles yield radiocarbon dates between 3820 and 3650 cal BC.

Before the Chassean culture, there are a few limited series with early Neolithic features dated between 4555 and $4460 \mathrm{cal}$ $\mathrm{BC}$ (fig. 23). The end of the Middle Neolithic sequence is represented by a site with no Chassean influence but which is still included in the group of smooth ceramics. It is dated between 3640 and 3520 cal BC (Monnet et al., 2002).

Alongside these ceramic styles, lithic industries (fig. 26 and 27) are characterized by the reduction of cobbles on cobbles (lithic style A), already present during the Early Neolithic period and associated with ceramic style 1. This type of reduction continues during style 2 and evolves towards early imports of unheated Bedoulian flint during style 2, partly in the form of blades (lithic style B). After $4000 \mathrm{cal} \mathrm{BC}$, bladelets in Bedoulian heated flint are present (lithic style $\mathrm{C}$ ), from ceramic style 3 b onwards. Lithic style $\mathrm{C}$ includes a variant (C1), which only concerns ceramic style $3 \mathrm{~b}$ and is closer to the semi-conical knapping method known in the Provence series (Binder, 1991; Léa, 2003). However, unlike for the latter, the pressure knapping platform is never faceted. The second variant $(\mathrm{C} 2)$, associated with ceramic styles 4 and 5, represents the flat quadrangular knapping method defined from the Provencal series (id. ibid.). Lastly, in the last Middle Neolithic phase, the networks of pale Bedoulian flint are abandoned.

It is difficult to correlate southern regions from the perspective of isotopic ages during the Middle Neolithic period (fig. 28) for a number of reasons: the qualitative and quantitative disparity of the ceramic series on which the chronologies are based; the increase in changes and/or cultural compartmentalisation; the plateau of the calibration curve that affects the dates between 4500 and 4000 cal BC, and lastly the lack of dates in reliable contexts. The Pre-Chassean elements in 
the Languedoc, Drôme, Provence and Liguria regions are no longer present by $4400 \mathrm{cal} \mathrm{BC}$ and the early Neolithic legacy is common to theses series. Segmented forms, multi-perforated handles, and plates or "vase-supports" with complex striped and chequered decoration are not yet present. The Middle Neolithic I is still difficult to grasp because of the lack of sites and paucity of the associated assemblages. It is difficult to understand why 400 years still escape us in the South when, in northern areas, the same period seems to be a buzzing cultural complex with a plethora of sites.

The subsequent period (the Proto-Chassean) is characterized by a better representation of smaller and more openshaped and thus thinner-walled ceramic containers (plates and bowls), a clear diversification of handle types and by the increase of "sub-cutaneous" perforated suspensions. Relief decoration becomes rare and gives rise to smooth pottery. In the Quercy, Languedoc and western Provence regions, ceramic forms are still not segmented and the first real Chassean elements appear irregularly depending on the sites. In contrast to results in eastern Languedoc, carinated recipients - mostly with a single handle - are already present in Liguria and Provence during this period, passed on from Italic cultures (Lepère, 2009; 2012; Luzi et Courtin, 2001). The absolute chronology of this period is difficult to grasp as radiocarbon dates are rare and incoherent. We estimate the end of the ProtoChassean at about $4250 \mathrm{cal}$ BC.

Available data on the early Chassean in the South of France vary greatly from one region to another. There are many sites in Languedoc, whereas the Provence, Drôme, and Quercy regions are characterized by the rarity and paucity of data. Villeneuve-Tolosane is the only reference for the Garonne river basin during this period (François, 2002; 2007). The prolonged character of this stage (at least 4300-4000 cal BC) can be partly explained by the fact that it is in the calibration curve plateau. Nevertheless, it is pertinent to ask whether this period could be sequenced, as shown by the presence of three variants $(2 \mathrm{a}$, $2 \mathrm{~b}$ and mixed). One of these variants $(2 \mathrm{~b})$ corresponds to the Les Plots style (Vaquer, 1990; 1991). However, there is marked stylistic consistency in the early southern Chassean, indicating a common tradition in the whole South of France, although other cultures have significant influence near the borders.

During more recent Chassean phases, processes of change and regionalisation seem to accelerate. At first, there is cultural continuity with the early Chassean period whereby numerous archaic elements in the Quercy, Garonnais, Provence and Liguria regions, as well as in style $3 \mathrm{a}$ in eastern Languedoc, are maintained. These elements are associated with new forms. This period, which can be dated between 4000 and 3900 cal $\mathrm{BC}$, includes the highest number of features classically attribu- ted to the Chassean culture. Some regional differences begin to appear, in particular between the Garonne and Quercy regions on the one hand, and Languedoc and Provence, on the other.

It is more difficult to classify the styles that follow this classical Chassean culture, both chronologically and geographically. Apart from later events at the end of the Middle Neolithic, all the recent styles seem to be part of the same time bracket, between 3900 and $3650 \mathrm{cal} \mathrm{BC}$.

Style-variant $3 \mathrm{~b}$ in eastern Languedoc can be cautiously compared with that of Provence. The archaic features have almost disappeared. Carinated types increase in number giving rise to new ceramic forms.

Style 4 in eastern Languedoc corresponds to the Auriac style, which extends from the eastern Pyrenees to Provence and the Drôme, but remains unknown in the Garonne river basin and Quercy, despite some affinities (Vaquer, 1990; 1991; Jédikian, 2000; Lepère, 2002; 2007; Léa et al., 2010).

Variants $5 \mathrm{a}$ and $5 \mathrm{~b}$ in eastern Languedoc and comparison styles, including the Bize group, highlight geographical differences. At least four areas stand out: Quercy and the Garonne river basin, where the most recent style extends into the Aude River basin (Cavanac; Vaquer, 1990; 1991) and indicates contact with the Atlantic, Pyrenees and Auvergne regions (François, 2002; 2007; Gernigon, 2004); central Languedoc where documentation on these phases remains very patchy; the banks of the Rhone (Vaquer, 1975; Timsit, 1994; Beeching, 1995; 2002; Lepère, 2009; 2012), which display strong style 5a cohesion and central Provence, where extra-cultural influences are clearly visible (Lepère, 2009).

The end of the Middle Neolithic is marked by series spread over the Rhône Valley and the nearby Provence coastal plain. They are dated between 3650 and 3500 cal BC. There is a gradation in their detachment from the Chassean tradition, but generally speaking, apart from carination, which does not completely disappear at the beginning of the Late Neolithic, very few elements are still present. Although these complexes are clearly set apart from the Chassean complex, they are still made up of smooth ceramics, which for us shows that they belong to the Middle Neolithic II.

The seriation proposed here for eastern Languedoc remains tenuous, especially for styles 1 and 4, which are only represented by a limited number of poorly-documented series. However, this work contributes to our knowledge of unpublished data, completes seriation research now available in the South of France and contributes to the definition of the Chassean culture. Collective work on the synchronization of southern sequencing can now be conducted in order to carry out further research on common grounds.

Translation: Louise Byrne 


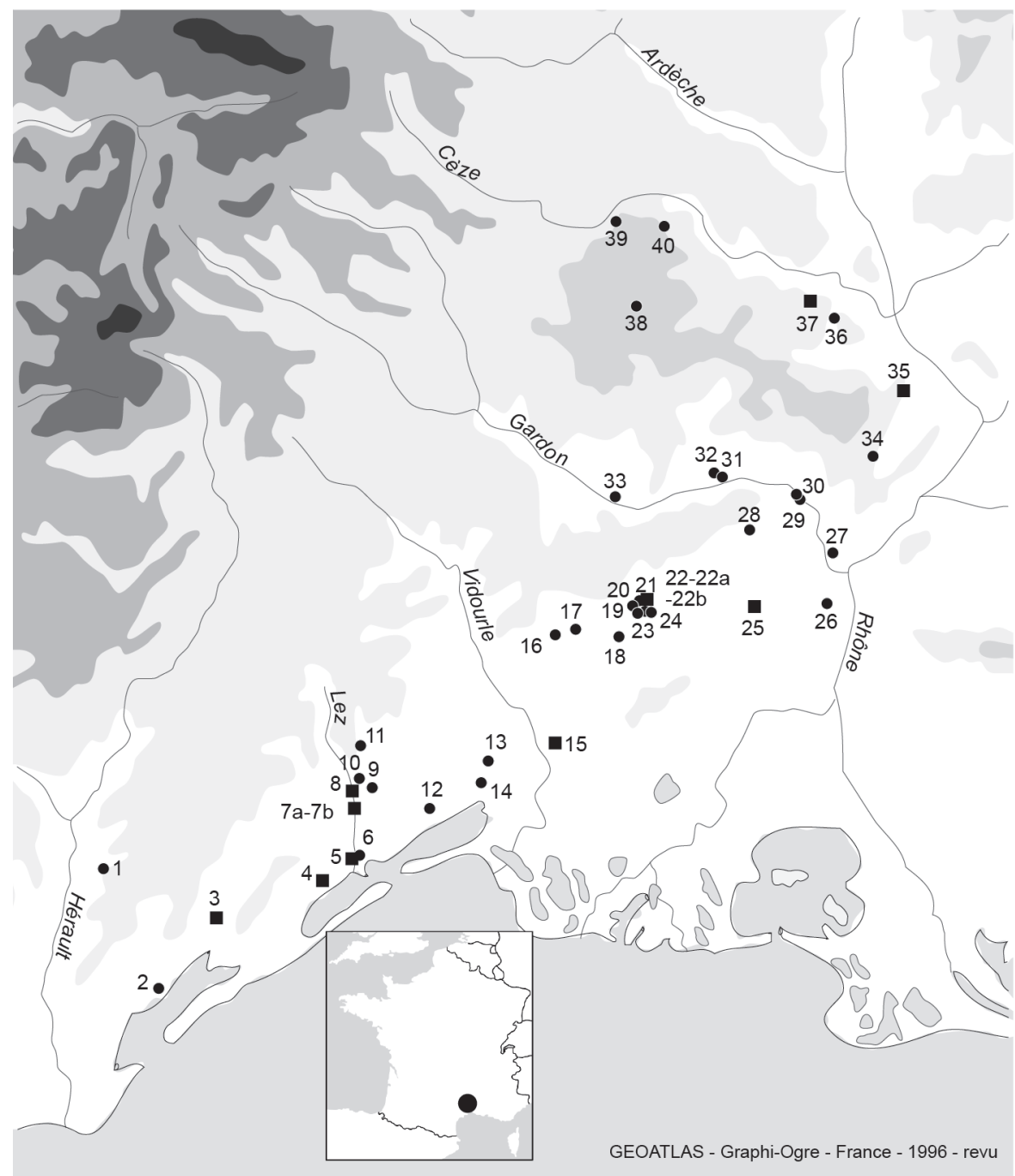

1 - Saint-Pargoire, Mas d'Embec (Hérault):

2 - Mèze, Mas de Guarric (Hérault)

3 - Gigean, La Clau III (Hérault);

4 - Villeneuve-lès-Maguelonne, grotte de la Madeleine (Hérault)

5 - Lattes - Port Ariane 3 (Hérault);

6 - Lattes, Filliès I (Hérault) :

7a - Montpellier, Plaine de Chrétien ;

$7 \mathrm{~b}$ - Jacques Coeur (Hérault);

8 - Castelnau-Le-Lez, Les Jardins de Vert Parc/

Le Moulin de Sauret (Hérault);

9 - Saint-Aunès - Saint Antoine/La Condamine (Hérault)

10 - Le Crès - Les Faysses Hérault)

11 - Teyran - Montbeyre-la-Cadoule

12 - Mauguio, La Capoulière 2 (Hérault);

13 - Lunel, Saint Jean de Nozé (Hérault)

14 - Saint-Nazaire-de-Pézan - L'Hournède (Hérault) ;

15 - Gallargues-Le-Montueux, Le Lavous (Gard)

16 - Congénies, Le Puech de la Fontaine (Gard)

17 - Nages-et-Sollorgues - Sur le Village

18 - Bernis, Les Faysses (Gard) ;

19 - Nîmes, Rue du Planas, Rue Edgar Mouton (Gard) :

20 - Nîmes - Mas des Abeilles II (Gard) :

21 - Nîmes, Voie Urbaine Sud (Gard);

22 - Nîmes, Mas de Vignole 4 ;

22a - Mas de Vignole 11 ;

22b - Mas Neuf et Cadereau d'Alès (Gard)

23 - Nîmes, Le Vistre de la Fontaine (Gard) ;

24 - Nîmes - Damier (Gard) ;

25 - Manduel, Fumérian (Gard);

26 - Jonquières-Saint-Vincent - Jarnègues (Gard) ;

27 - Cabrières - Baume Bourbon (Gard)

28 - Montfrin - Le Réal (Gard)

29 - Remoulins - grotte Féraud (Gard)

30 - Remoulins - grotte du Taï ;

31 - Sainte-Anastasie, grotte Saint Joseph (Gard) ;

32 - Sainte-Anastasie - Baume Latrone (Gard)

33 - Sanilhac-Sagriès, grotte de Saint Vérédème (Gard)

34 - Rochefort-du-Gard, La Grange des Merveilles I (Gard)

35 - Roquemaure - La Ramière (Gard)

36 - Tresques - La Roquette (Gard) ;

37 - Cavillargues - La Roquette (Gard)

38 - Seynes - grotte des Trois Ours (Gard);

39 - Tharaux - grotte des Fées (Gard);

40 - Mejanes-le-Clap - grotte de la Citerne

Fig. 1 - Localisation des principaux gisements du Languedoc oriental ; les carrés indiquent ceux qui ont directement servi aux analyses.

Main chassean sites of Eastern Languedoc; squares indicate those that were directly used in the statistical analysis.

\section{INTRODUCTION}

La sériation des corpus céramiques du Chasséen méridional s'est enrichie ces dix dernières années en grande partie grâce à l'aboutissement des travaux de thèse sur le Garonnais (François, 2002 ; 2007 ; Gandelin, 2011), le Quercy (Gernigon, 2004) et la Provence (Lepère 2009 ; 2012). Ces travaux représentent un progrès certain par rapport à ceux des années 80-90, en tant qu'ils se sont attachés à l'analyse méthodique, critique et exhaustive de corpus de grande ampleur. Auparavant, les périodisations de J. Vaquer $(1990 ; 1991)$ et de A. Beeching (1995) avaient force de loi dans le domaine du Chasséen méridional. Elles ne sont pas pour autant obsolètes. Reposant sur des intuitions et des observations limitées, plus ou moins extrapolées, elles ont permis d'établir un cadre qui n'est pas totalement remis en cause mais qui a tout de même évolué.

Dans ce contexte, le Languedoc oriental se présente comme le maillon manquant de la trame méditerranéenne des périodisations chasséennes de la céramique, puisqu'il n'a bénéficié jusqu'ici que d'un article à la portée limitée
(Georjon, 2003) ${ }^{1}$. Il est d'autant plus important de proposer un travail plus abouti sur les mobiliers céramiques chasséens de cette région que les industries lithiques associées ont fait l'objet d'une thèse (Léa, 2004a).

Cet article propose donc une périodisation des mobiliers céramiques ${ }^{2}$ fondée sur une sériation automatique et sur une analyse factorielle des correspondances. Les groupes ainsi obtenus sont replacés dans le cadre des périodisations méridionales existantes. Ce travail est assorti d'une révision de la chronologie radiocarbone disponible et d'une tentative d'har-

1. Cet article de 2003 ainsi que le présent article se réfèrent à la thèse de C. Georjon, initiée en 1999 et inachevée.

2. Les données céramiques et lithiques inédites sont issues d'une fouille programmée (La Roquette à Cavillargues, Gard, dirigée par H. Petitot), du réexamen de collections anciennes, de nos participations aux rapports de fouille de l'INRAP et de notre engagement dans le Projet Collectif de Recherche (PCR) « Espace rural et occupation du sol de la région nîmoise de la Préhistoire à l'époque moderne » dirigé par J.-Y. Breuil. Par ailleurs, nous remercions J.-Y. Breuil, J. Cauliez, A. Gaillard, K. Gernigon, A. Hasler et C. Lepère. 
monisation des périodisations concernant la céramique et les industries lithiques taillées. Enfin, une discussion plus générale sera engagée concernant la place du Languedoc oriental dans le Chasséen méridional.

\section{LE CADRE DE L'ÉTUDE}

Le Languedoc oriental, logé entre le cours du Rhône, de l'Ardèche et de l'Hérault, est composé de trois entités naturelles étagées suivant un axe $\mathrm{SO} / \mathrm{NE}$ qui sont, du plus loin au plus près de la mer, la retombée des Causses, les garrigues du piémont et la plaine littorale. La grande majorité des sites du Néolithique moyen sont localisés dans la plaine (fig. 1).

Si le nombre de gisements du Néolithique moyen est conséquent dans cette région, dès qu'il s'agit de sérier le mobilier, on ne peut compter que sur quelques-uns d'entre eux.

Le Néolithique moyen non chasséen n'est représenté que sur un nombre très restreint de sites. Pour la période ante-chasséenne, les séries sont indigentes et se concentrent dans le nîmois ; pour la période post-chasséenne, un seul ensemble est connu dans le nord du Gard (La Grange des Merveilles à Rochefort-du-Gard). Ces éléments n'ont pas été injectés dans les analyses statistiques et seront traités dans un chapitre à part.

Concernant le Chasséen, la nécessité actuelle de resserrer les chronologies incite à éliminer d'emblée nombre de séries qui, quoique conséquentes, ne remplissent pas les conditions requises pour une telle entreprise. C'est le cas des ramassages de surface du Puech de la Fontaine (Roger et Valette, 1988), des sondages de Filliès 1 (port antique) de Lattes (Mendoza et Prades, 1988), de la stratigraphie problématique de Montbeyre la Cadoule à Teyran (Arnal et al., 1991) et de la grotte de la Madeleine, fouilles Arnal (Arnal, 1956), sans oublier bien sûr les riches collections provenant des grottes gardoises (Louis, 1953 ; Vaquer, 1975 ; Timsit, 1994). C'est également le cas des séries trop restreintes malgré des contextes fiables : L'Hournède à Saint-Nazaire-de-Pézan, Les Iragnons-Perrier à Codognan, La Capoulière 2 à Mauguio, La Roquette à Tresques, Le Réal à Montfrin, etc. (Marchand et Vaquer, 1976 ; Bordreuil et Roger, 1992 ; Georjon, 2000 ; inédit, étude C. Georjon ; Noret, 2002). Ajoutons enfin que certaines collections plus ou moins importantes, bien qu'étant peut-être exploitables dans cette optique, n'ont pu faire l'objet d'un réexamen jusqu'à présent : Raffègues à Mèze (Montjardin et Rouquette, 1988), Puech de la Fontaine à Congénies, Baume Bourbon à Cabrières (inédit, fouille J.-L. Roudil), Jarnègues à Jonquières-Saint-Vincent (Bazile et al., 2002) notamment. Certains de ces ensembles pourront être intégrés dans un deuxième temps par rapport à la sériation que nous proposerons plus avant.

\section{ANALYSE DU CORPUS CÉRAMIQUE CHASSÉEN}

\section{LE CHOIX DES ENSEMBLES ET DES VARIABLES}

Le corpus pris en compte dans cette analyse (tabl. I) est composé de 12 sites pour la plupart inédits, répartis dans la plaine littorale et la basse vallée du Rhône. À titre de comparaison, nous y avons adjoint trois ensembles céramiques issus de deux sites provençaux et un dernier issu d'un site du Languedoc occidental.

L'unité de base de la plupart des ensembles céramiques retenus est la structure en creux. On peut estimer par postulat que leur période de remplissage n'excède pas une à deux générations, soit au maximum 70-80 ans, ce qui en fait une unité de temps relativement acceptable si aucune perturbation majeure n'est à déplorer. Deux gisements stratifiés ont également été retenus, Port Ariane à Lattes et la grotte de la Madeleine à Villeneuve-lès-Maguelonne, fouille L. Barral. L'analyse minutieuse et pluridisciplinaire de la stratigraphie de Port Ariane (Davaux, 2007) ne laisse aucun doute sur sa fiabilité. Les deux nappes de mobilier superposées (21077 et 21078) ont livré des éléments très cohérents. Le mobilier pris en compte dans ces analyses est issu de ces deux nappes et de foyers qui s'ouvrent à la surface de ces nappes, l'ensemble ayant été considéré comme un tout homogène. La stratigraphie de la grotte de La Madeleine est a priori remaniée dans sa majeure partie. Les fouilles de L. Barral qui, dans les années 1950, prenaient la suite de celles de J. Arnal, avaient pour objectif de trouver un endroit où la stratigraphie était encore en place en s'aidant de la géomorphologie, discipline alors rarement usitée en archéologie (Barral, 1960). Le récent travail réalisé sur les mobiliers lithiques et céramiques de la collection de L. Barral (Léa, 2004a ; inédit, étude Georjon), montre que ce fouilleur avait atteint le but qu'il s'était fixé puisqu'une évolution chrono-culturelle est sensible entre la base et le sommet de la stratigraphie. Les couches IX à VII, les plus anciennes, n'ont cependant pas pu être prises en compte dans les analyses qui suivent en raison d'un trop faible nombre de pièces.

Trente-huit ensembles, composés de faits et d'unités stratifraphiques, ont ainsi été pris en compte dans les analyses qui suivent.

Le corpus des variables est composé de 31 éléments (fig. 2). Il s'agit de formes de récipients restituables et d'éléments de typologie fragmentaires (formes de bords, de cols et de rupture de pente, éléments de préhension et de décors). Chacun de ces types se retrouve dans trois ensembles céramiques au minimum ; ils sont supposés signifiants sur le plan chrono-culturel. Dans le cadre de ce travail, nous avons choisi de rejeter les éléments dont la variabilité est difficile à faire ressortir (majorité des formes simples, des boutons et languettes de préhension).

Quatre catégories de récipients, chaque fois déclinées en deux variantes en fonction de leur volume, sont reconnues et définies par les rapports suivants :

- assiettes et plats : DM (Diamètre Moyen) > 2,5 x HT

(Hauteur Totale) ;

- coupes et jattes : 2,5 x HT > DM > 1,5 x HT ;

- bols et marmites : 1,5 x HT > DM (Hauteur Totale sur

Diamètre Moyen $>$ HT ;

- gobelets et jarres : DM $<$ HT.

Leur profil peut être continu, infléchi, sinueux, à col ou segmenté (par une carène, un ressaut ou un épaulement). Etant donné la variété des types de récipients segmentés, une discrimination supplémentaire est fondée sur le double rapport 
Tabl. I - effectifs des ensembles céramiques analysés.

Staff of the analyzed ceramic sets.

\begin{tabular}{|c|c|c|c|c|c|c|c|}
\hline Dpt & Nom du site & $\begin{array}{l}\text { Nom } \\
\text { du fait }\end{array}$ & Type & Code & $\begin{array}{l}\text { Nombre } \\
\text { de tessons }\end{array}$ & $\begin{array}{c}\text { Nombre } \\
\text { d'éléments } \\
\text { diagnostics }\end{array}$ & Références \\
\hline \multirow{20}{*}{ 일 } & $\begin{array}{l}\text { Gallargues-le-Montueux - Le } \\
\text { Lavous - plein air }\end{array}$ & Fc. Ilb & fosse & Lv & $?$ & 12 & $\begin{array}{l}\text { Fenouillet et Vaquer, } \\
1974\end{array}$ \\
\hline & Manduel - Fumérian - plein air & F1092 & fosse & $\mathrm{Fm}$ & 67 & 12 & $\begin{array}{l}\text { Inédit, responsable A. } \\
\text { Hasler, étude C. Georjon }\end{array}$ \\
\hline & \multirow{2}{*}{$\begin{array}{l}\text { Roquemaure - La Ramière - } \\
\text { plein air }\end{array}$} & F5083 & fosse & $\mathrm{Rm} 1$ & 125 & 31 & \multirow[t]{2}{*}{ Georjon, 2002} \\
\hline & & F8048 & foyer en cuvette & $\mathrm{Rm} 2$ & 77 & 16 & \\
\hline & \multirow{5}{*}{$\begin{array}{l}\text { Cavillargues - La Roquette - } \\
\text { plein air }\end{array}$} & F1439 & fosse & $\mathrm{Rq} 1$ & 371 & 83 & \multirow{5}{*}{$\begin{array}{l}\text { Inédit, responsable } \\
\text { H. Petitot, } \\
\text { étude C. Georjon }\end{array}$} \\
\hline & & F1645 & fosse & $\mathrm{Rq} 2$ & 181 & 54 & \\
\hline & & F1540 & fosse & $\mathrm{Rq} 3$ & 271 & 54 & \\
\hline & & F1585 & fosse & $\mathrm{Rq} 4$ & 379 & 62 & \\
\hline & & F1361 & fosse & Rq5 & 330 & 55 & \\
\hline & \multirow{4}{*}{$\begin{array}{l}\text { Nîmes - Mas de Vignole IV - } \\
\text { plein air }\end{array}$} & F2123 & fosse & Mv1 & 7 & 7 & \multirow{4}{*}{$\begin{array}{l}\text { Inédit, responsable } \\
\text { L. Jallot, } \\
\text { étude C. Georjon }\end{array}$} \\
\hline & & F8013 & fosse & Mv2 & 97 & 12 & \\
\hline & & F8056 & fosse & Mv3 & 629 & 57 & \\
\hline & & F4094 & fosse & Mv5 & 260 & 26 & \\
\hline & $\begin{array}{l}\text { Nîmes - Mas de Vignole } 11 \text { - } \\
\text { plein air }\end{array}$ & F1128 & fosse & Mv4 & 92 & 17 & $\begin{array}{l}\text { Inédit, responsable } \\
\text { P. Séjalon, } \\
\text { étude C. Georjon }\end{array}$ \\
\hline & \multirow{6}{*}{$\begin{array}{l}\text { Nîmes - Bassin aval du Cade- } \\
\text { reau d'Alès - plein air }\end{array}$} & F1094 & fosse & $\mathrm{Cd} 1$ & 324 & 28 & \multirow{6}{*}{$\begin{array}{l}\text { Inédit, responsable } \\
\text { A. Hasler, } \\
\text { étude C. Georjon }\end{array}$} \\
\hline & & F3162 & fosse & $\mathrm{Cd} 2$ & 871 & 75 & \\
\hline & & F5129 & fosse & $\mathrm{Cd} 3$ & 394 & 81 & \\
\hline & & F1055 & fosse & $\mathrm{Cd} 4$ & 178 & 46 & \\
\hline & & F1043 & fosse & Cd5 & 366 & 30 & \\
\hline & & F1063 & fosse & Cd6 & 165 & 14 & \\
\hline \multirow{14}{*}{$\begin{array}{l}\frac{ \pm}{5} \\
\frac{\pi}{\frac{\pi}{\pi}} \\
\frac{\pi}{I}\end{array}$} & \multirow{3}{*}{$\begin{array}{l}\text { Castelnau-le-Lez - Les Jardins } \\
\text { de Vert Parc - plein air }\end{array}$} & F1068 & fosse & Jv1 & 300 & 52 & \multirow[t]{3}{*}{ Georjon, 2003} \\
\hline & & F1095 & fosse & Jv2 & 87 & 15 & \\
\hline & & F1020 & puits & Jv3 & 200 & 44 & \\
\hline & \multirow{2}{*}{$\begin{array}{l}\text { Montpellier - Jacques Cœur } 2 \text { - } \\
\text { plein air }\end{array}$} & F22 & dépôt d'objets & Jc1 & 134 & 24 & \multirow{2}{*}{$\begin{array}{l}\text { Jallot et al., } 2000 \text {; } \\
\text { Georjon, } 2003\end{array}$} \\
\hline & & F9 & fosse & Jc2 & 58 & 14 & \\
\hline & $\begin{array}{l}\text { Montpellier - Plaine de Chrétiens } \\
\text { - plein air }\end{array}$ & F39 & puits & $\mathrm{Pc}$ & 371 & 92 & Georjon, 2003 \\
\hline & $\begin{array}{l}\text { Lattes - Port Ariane III - plein air } \\
\text { stratifié }\end{array}$ & $\begin{array}{l}21078 \text { et } \\
21077\end{array}$ & $\begin{array}{l}\text { couches sédimentaires } \\
\text { à mobilier homogène }\end{array}$ & Lp & 2043 & 141 & Georjon, 2007 \\
\hline & \multirow[t]{2}{*}{ Gigean - La Clau - plein air } & F1062 & fosse & $\mathrm{Cl} 1$ & 240 & 24 & \multirow{2}{*}{$\begin{array}{l}\text { Inédit, responsable } \\
\text { A. Hasler, } \\
\text { étude C. Georjon }\end{array}$} \\
\hline & & F1195 & fosse & $\mathrm{Cl} 2$ & 130 & 19 & \\
\hline & \multirow{5}{*}{$\begin{array}{l}\text { Villeneuve-lès-Maguelonne - La } \\
\text { Madeleine - grotte stratifié }\end{array}$} & c.VI & couche sédimentaire & Md1 & 455 & 71 & \multirow{5}{*}{$\begin{array}{l}\text { Barral, } 1960 \text { et inédit, } \\
\text { étude C. Georjon }\end{array}$} \\
\hline & & c.V & couche sédimentaire & Md2 & 460 & 104 & \\
\hline & & c.IV & couche sédimentaire & Md3 & 463 & 80 & \\
\hline & & c.III & couche sédimentaire & Md4 & 1263 & 321 & \\
\hline & & c.I/II & couche sédimentaire & Md5 & 1339 & 384 & \\
\hline \multirow{3}{*}{ 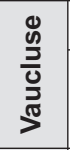 } & Piolenc - Rocalibert - plein air & niv & niveau archéologique & Rc & 695 & 140 & Léa et al., 2004 \\
\hline & \multirow{2}{*}{$\begin{array}{l}\text { L'Isle-sur-La-S. - Les Bagnoles } \\
\text { - plein air }\end{array}$} & F48 & fosse & $\mathrm{Bg} 1$ & 225 & 25 & \multirow[t]{2}{*}{ Sargiano et al., 2010} \\
\hline & & F75 & fosse & Bg2 & 261 & 42 & \\
\hline$\frac{0}{\frac{0}{2}}$ & $\begin{array}{l}\text { Carcassonne - Le Champ du } \\
\text { Poste - plein air }\end{array}$ & F139 & fosse & $\mathrm{Cp}$ & 1404 & 58 & $\begin{array}{l}\text { Inédit, responsable } \\
\text { F. Convertini, } \\
\text { étude C. Georjon }\end{array}$ \\
\hline
\end{tabular}


$\mathrm{HT} / \mathrm{DM}$ et $\mathrm{HC} / \mathrm{HT}^{3}$, généralement signifiant pour ces formes (Vaquer, 1975 ; Jédikian, 2000).

Le choix des éléments de préhension et de décor répond d'abord à la nécessité de les retrouver dans au moins 3 ensembles analysés. Ainsi les décors sont largement sous-représentés car ils sont rares et variés.

\section{SÉRIATION AUTOMATIQUE}

La sériation automatique a été réalisée en présence/absence des variables, compte tenu de la disparité des effectifs (fig. 3). On remarque d'emblée qu'une diagonale apparaît. Une césure forte s'établit vers le centre du tableau, caractérisée par la multiplication soudaine des variables en présence.

Le haut du tableau (groupe 1) est composé de huit ensembles gardois (Lv, Fm, Mv1, Mv2, Mv4, Cd1, Cd2, Cd3), de huit ensembles héraultais (Lp, Jv1, Jv2, Jc1, Jc2, Lp, Cl1, $\mathrm{Cl} 2)$, de deux séries vauclusiennes $(\mathrm{Bg} 1, \mathrm{Bg} 2)$ et d'une série audoise (Cp). On constate l'homogénéité importante de cette partie du tableau. Les variables associées sont celles qui caractérisent traditionnellement les phases anciennes du Chasséen.

Le bas du tableau est plus hétérogène. Deux parties sont plus ou moins visibles (groupes $2 \mathrm{a}$ et $2 \mathrm{~b}$ ). Le groupe $2 \mathrm{a}$ est caractérisé par une dilation importante des critères dont plusieurs sont des survivances des phases anciennes du Chasséen représentées par le groupe 1. Il se distingue par quatre ensembles gardois (Mv3, Cd4 à 6) et cinq ensembles héraultais tous issus de la grotte de la Madeleine (Md1 à 5). Le groupe 2b montre une nouvelle réduction des occurrences avec un net recul des types anciens. Il comprend sept ensembles gardois (Rm1 et 2, Mv5, Rq1 à 4), deux ensembles héraultais (Jv3, Pc) et une série vauclusienne (Rc).

Opérer un découpage plus poussé de cette sériation automatique nous paraît hasardeux. Nous avons donc choisi de procéder à une analyse factorielle des correspondances qui livrera un découpage moins empirique.

\section{ANALYSE FACTORIELLE DES CORRESPONDANCES}

Pour ce travail, toutes les données de la sériation automatique ont été conservées ; elles sont également analysées en présence-absence.

Le premier plan factoriel représente $30,79 \%$ de l'inertie, ce qui est bien faible; le pourcentage chute rapidement à partir de F2 mais les cinq premiers axes ne cumulent que $55 \%$ de l'information. Cela s'explique en grande partie par l'étendue du tableau de contingence.

La dilatation des données permet une bonne approche. On remarque d'emblée que trois grands ensembles se dégagent (fig. 4). Sur le premier axe s'opposent deux groupes d'ensembles avec les plus fortes contributions relatives; d'une part $\mathrm{Cd} 2$ et $\mathrm{Bg} 2$ et de l'autre Rq1 et Rc. Sur le second axe, s'opposent de la même manière Md4 avec Rq3.

3. Hauteur de la rupture de pente sur hauteur totale
La classification ascendante hiérarchique (CAH) a été réalisée avec la méthode d'agrégation de Ward sur les coordonnées principales des lignes. Elle permet d'identifier 6 classes (fig. 5). Lorsqu'on repositionne sur le plan de l'AFC (fig. 6), on remarque que les classes 3, 5 et 6 sont relativement indépendant les unes des autres, tandis que les classes 1,2 et 5 sont agglutinées à droite du plan factoriel.

\section{Les Classes 3, 5 et 6}

Les classes formées à gauche du graphique (classes 3, 5 et 6) rassemblent la majorité des variables analysées.

La classe 3, située en haut à gauche du graphique, se distingue principalement par la variété des types de récipients segmentés (F4, F7 et F9), par les carènes à ressaut, les plaquettes multiforées (P5), les sillons internes multiples (D2) et les décors de moustaches (D3). Elle rassemble toutes les séries de la grotte de La Madeleine à Villeneuve-les-Maguelonne dans l'Hérault (Md1 à 5), 3 ensembles du Cadereau d'Alès à Nîmes (Cd4 à 6) et un du Mas de Vignole à Nîmes (Mv3).

La classe 6 partage une partie des variables de la classe 3 et se distingue essentiellement par les sillons multiples (D2). Elle comprend les deux ensembles de la Ramière à Roquemaure dans le Gard (Rm1 et 2) et Plaine de Chrétien-F39 à Montpellier (Pc).

La classe 5 , en bas à gauche du graphique, rassemble cinq variables à forte contribution relative $(\mathrm{C} 1, \mathrm{R} 3, \mathrm{~F} 5, \mathrm{~F} 6$ et P6). Les types de formes segmentées sont diversifiées (F5, F6 et F10) et s'accompagnent des cols déversés de coupes carénées $(\mathrm{C} 1)$, des inflexions appuyées sur coupes larges (R3), des carénés à cran interne (C2) et des rubans multiforés (P6). Elle concerne Rocalibert à Piolenc dans le Vaucluse (Rc), tous les ensembles de La Roquette à Cavillargues dans le Gard (Rq), un ensemble du Mas de Vignole 4 à Nîmes (Mv5) et des Jardins de Vert Parc à Castelnau-le-Lez dans l'Hérault (Jv3).

Ces trois classes se composent de variables traditionnellement associées aux phases récentes du Chasséen. La classe 5 présente l'autonomie la plus forte tandis que les classes 3 et 6 ont davantage de relations. La comparaison de ces résultats avec la sériation automatique offre une bonne adéquation. La classe 3 correspond au groupe $2 \mathrm{a}$; la classe 5 au groupe $2 \mathrm{~b}$. La position de ces deux classes ou groupes s'explique assez facilement par la chronologie : tandis que le groupe 2 a ou classe 3 conserve des variables issues du Chasséen ancien quoique sporadiques (anses en ruban, boutons quadrangulaires, tubulures horizontales, bourrelet/bandeau pré-oral interne sur coupe, bandeau pré-oral externe sur jarre, essentiellement), le groupe $2 \mathrm{~b}$ ou classe 5 s'en affranchit presque totalement.

Les trois ensembles de la classe 6 sont insérés dans le groupe $2 \mathrm{~b}$ de la sériation automatique, seule l'AFC les distingue. On retiendra que les deux ensembles de La Ramière (Rm1 et $\mathrm{Rm} 2$ ) sont restreints tandis que la série de Plaine de Chrétien (Pc) montre, malgré son ampleur, une faible variabilité typologique. Elle se compose pour l'essentiel de deux types de formes récurrentes, ce qui ne peut manquer d'être interprété comme un biais fonctionnel. Quoiqu'il en soit, les deux analyses pratiquées - sériation automatique et $\mathrm{AFC}$ - font ressortir le manque d'indépendance de la classe 6 qui se présenterait comme un intermédiaire entre les classes 3 et 5 . 


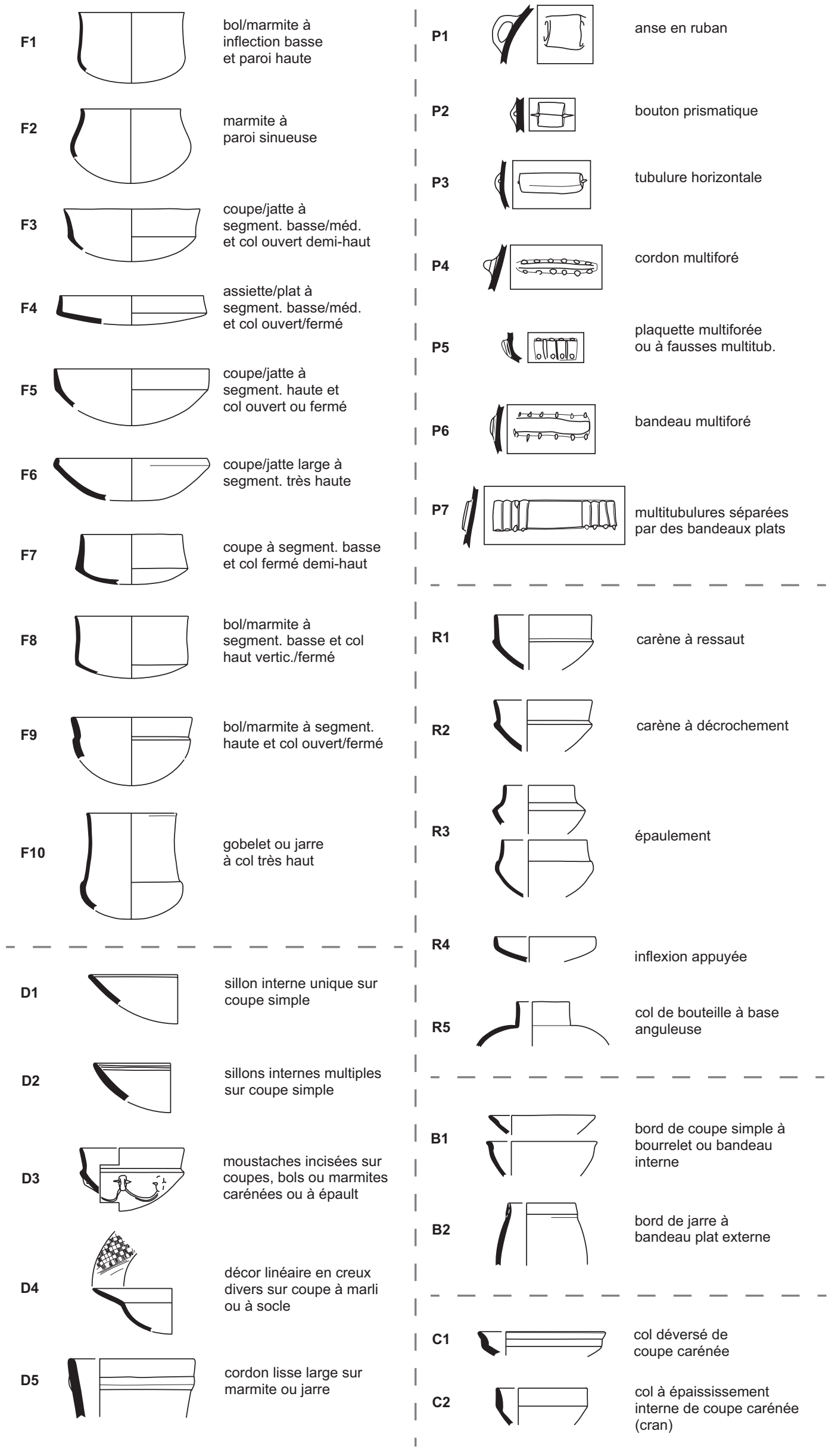


4 Fig. 2 - Les variables typologiques retenues pour les analyses. Éléments de bord (B), de col (C), de rupture de pente $(R)$, de forme $(F)$, de préhension $(P)$ et de décor $(D)$.

Typological criteria selected for the analysis. Elements of edge $(B)$, neck (C), break (R), form (F), grip (P) and decoration (D).

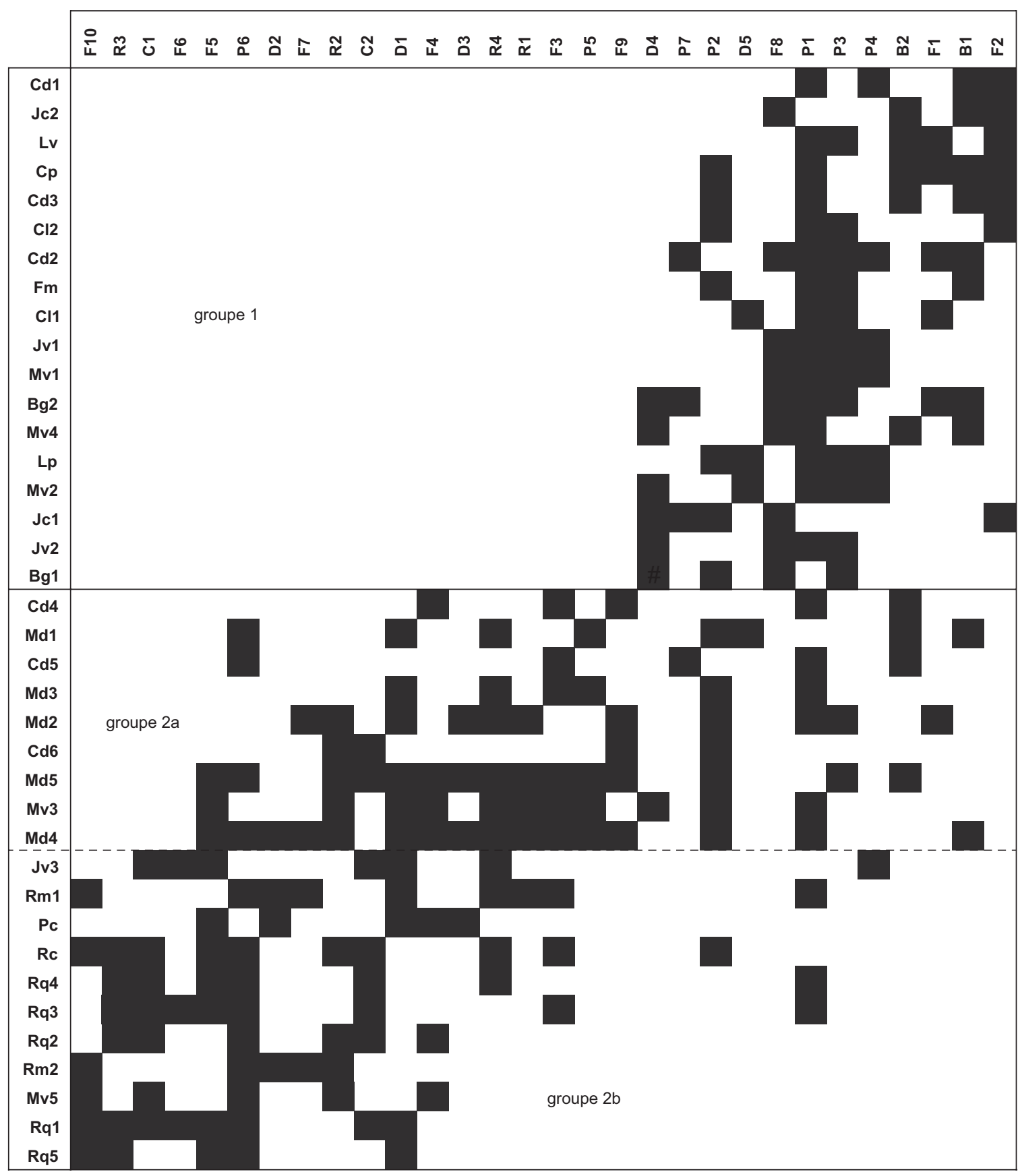

Fig. 3 - Sériation automatique des ensembles céramiques analysés (sériographe 0.2 Excel Bruno Desachy 2002-2004). 
Graphique symétrique

(axes F1 et F2:30,79\%)

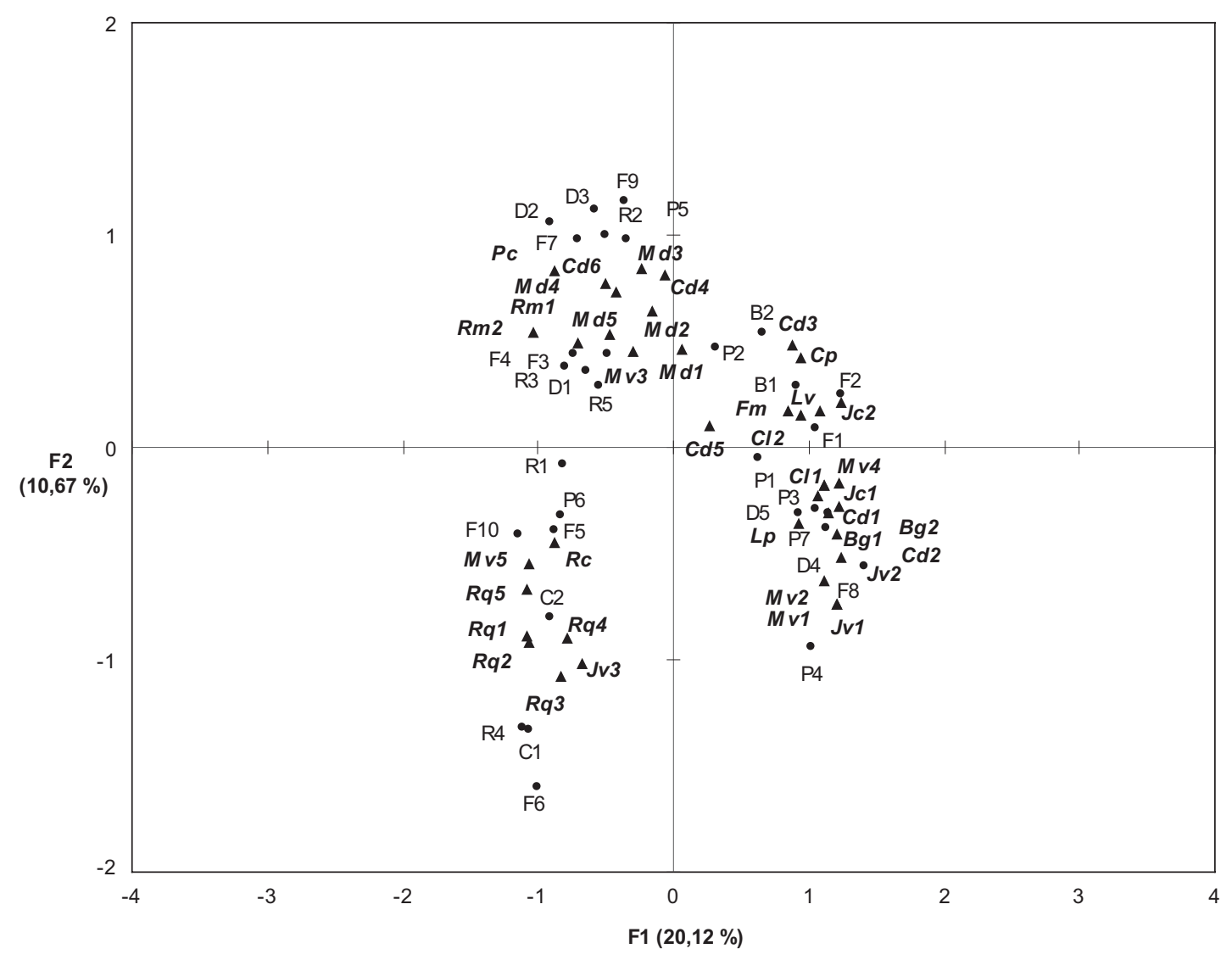

Fig. 4 - Analyse factorielle des correspondances des séries chasséennes.

Correspondence analysis of chassean series.

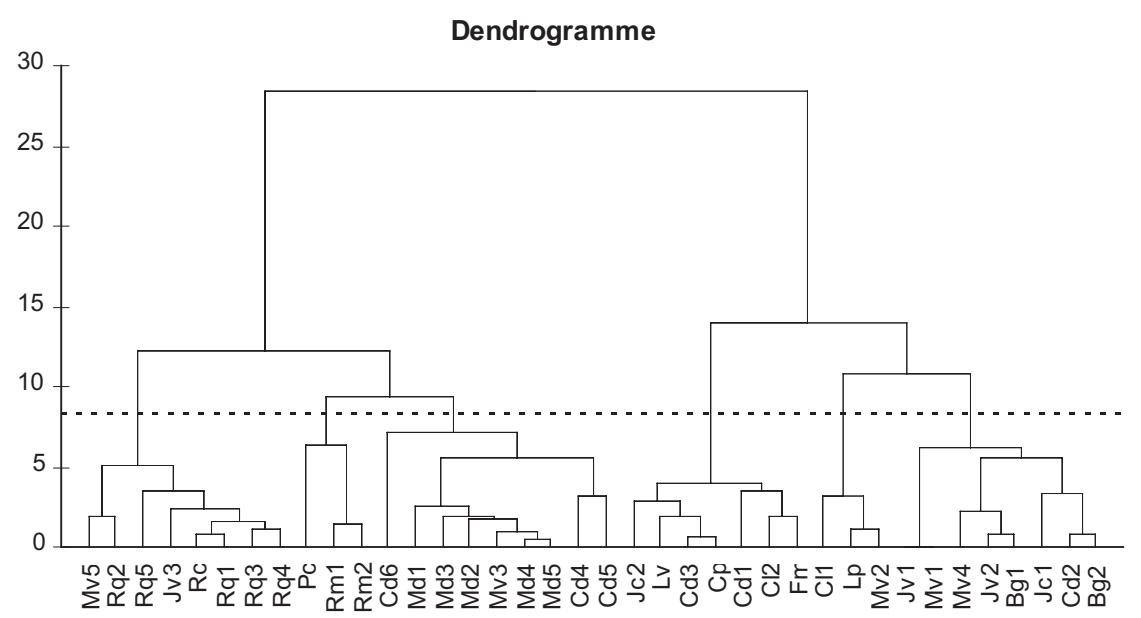

Fig. 5 - Classification ascendante hiérarchique réalisée selon la méthode de Ward sur les coordonnées principales des lignes de l'analyse factorielle des correspondances des séries chasséennes du Languedoc oriental.

Cluster analysis (method of Ward) carried on the main coordinates of lines . 


\section{Graphique symétrique \\ (axes F1 et F2 : 30,79\%)}

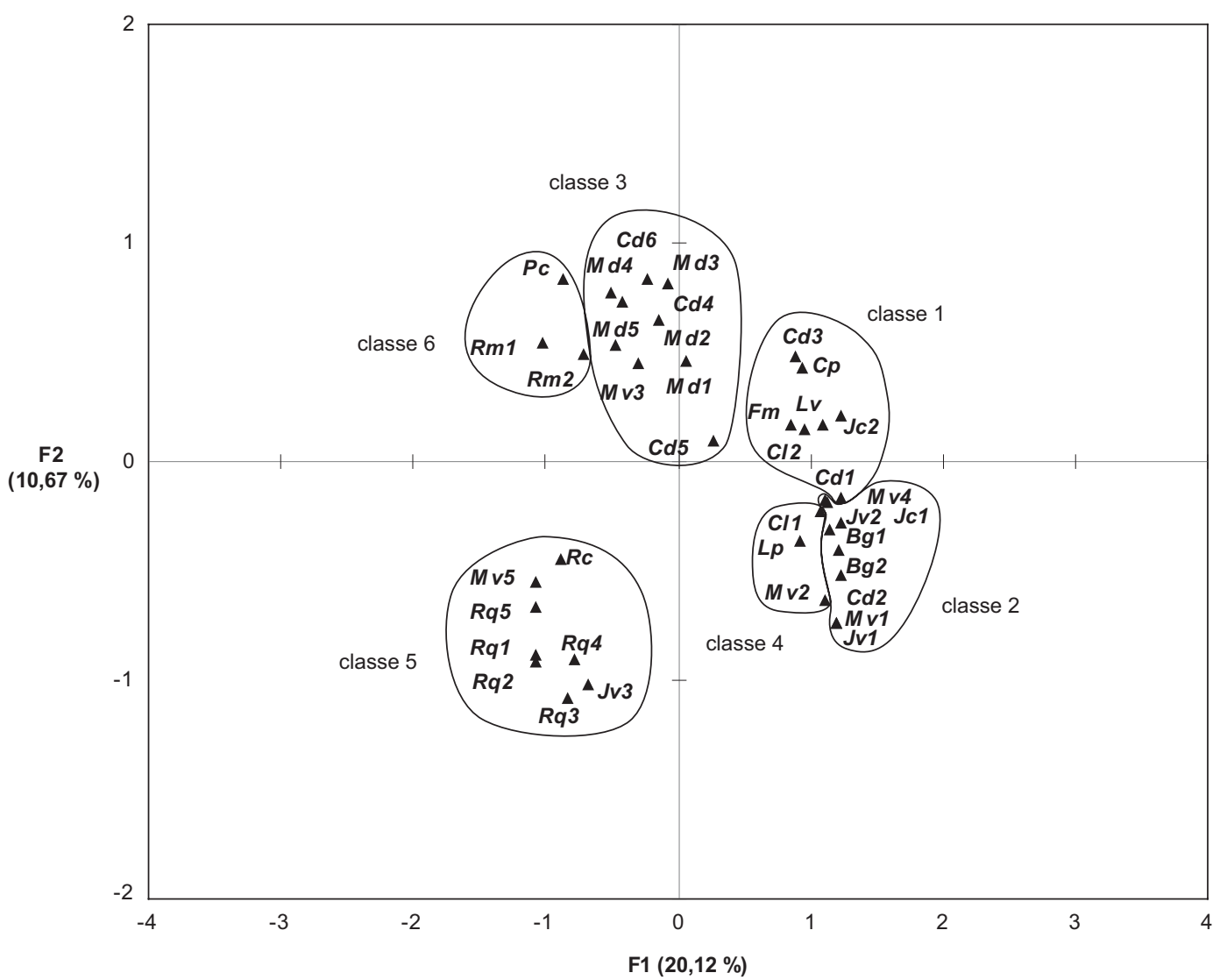

Fig. 6 - Report des classes de la CAH sur l'AFC des séries chasséennes

Classes of the cluster analysis to the graph.

\section{Les Classes 1, 2 et 4}

Le grand amas à droite du graphique de l'AFC rassemble toutes les variables traditionnellement associées aux phases anciennes du Chasséen. La contribution relative de certaines d'entre elles est importante (B2, F2, F8, P1 et P3). L'imbrication des trois classes (classes 1, 2 et 4), qui partitionnent cet agrégat, montre qu'elles ne sont pas autonomes. Les ensembles concernés sont globalement plus fragmentés et donc moins bien caractérisés typologiquement que les ensembles récents qui concentrent le plus grand nombre de variables.

Afin de clarifier la position des ensembles anciens, on a procédé à une analyse factorielle complémentaire les concernant sans rien changer aux données de l'AFC précédente (fig. 7).

L'inertie des axes 1 et 2 passe à 45,5\%. La chute du pourcentage est rapide après l'axe 2 et les 4 premiers axes cumulent plus de $70 \%$ de l'information.

Les données sont toutes regroupées près du centre des axes. À première vue, aucun groupe ne se dégage franchement, cependant les contributions montrent des oppositions. Sur l'axe 1, elles concernent Cd3, Jc2 et Cp d'une part, et Lp et Mv2 d'autre part. Sur l'axe 2 s'opposent Jc1, Jv2, Bg1 et Bg2 à Lp.

Les classes de la CAH se distinguent un peu plus clairement mais demeurent dépendantes les unes des autres (fig. 8).
La classe 1, en bas à droite du graphique, rassemble Le Lavous-couche IIb (Lv), Fumérian-F1092 (Fm), deux ensembles du Cadereau d'Alès à Nîmes $(\mathrm{Cd} 1, \mathrm{Cd} 3)$, un ensemble de Jacques Cœur 2 (Jc2), une série de La Clau à Gigean dans l'Hérault (Cl2) et Le Champ du Poste-F139 (Cp). Les variables associées sont principalement les bourrelets/ bandeaux pré-oraux internes sur coupes (B1) et les jarres à bandeau pré-oral externe (B2), les marmites à inflexion basse (F1) et celles à paroi sinueuse (F2). On notera que Jc2 est un peu à l'écart de la classe 1 ; il se positionne en intermédiaire entre les classes 1 et 2.

La classe 2, en haut du graphique se compose d'une série du Cadereau d'Alès (Cd2), du Mas de Vignole 11 (Mv4), du Mas de Vignole IV (Mv1) et de Jacques Cœur 2 (Jc1), des deux ensembles des Jardins de Vert Parc (Jv1, Jv2) et des deux ensembles du site vauclusien des Bagnoles (Bg1, Bg2). Elle se caractérise par les coupes et marmites à carène basse et paroi haute (F8), les multitubulures (P7) et les décors sur coupes à marli ou à socle (D4).

La classe 4 s'isole un peu plus nettement des autres en bas à gauche du plan. Elle comprend Port Ariane (Lp), une série du Mas de Vignole 4 (Mv2) et de La Clau III (C11) et se caractérise essentiellement par les décors de cordons lisses larges (D5) et les cordons multiforés. 


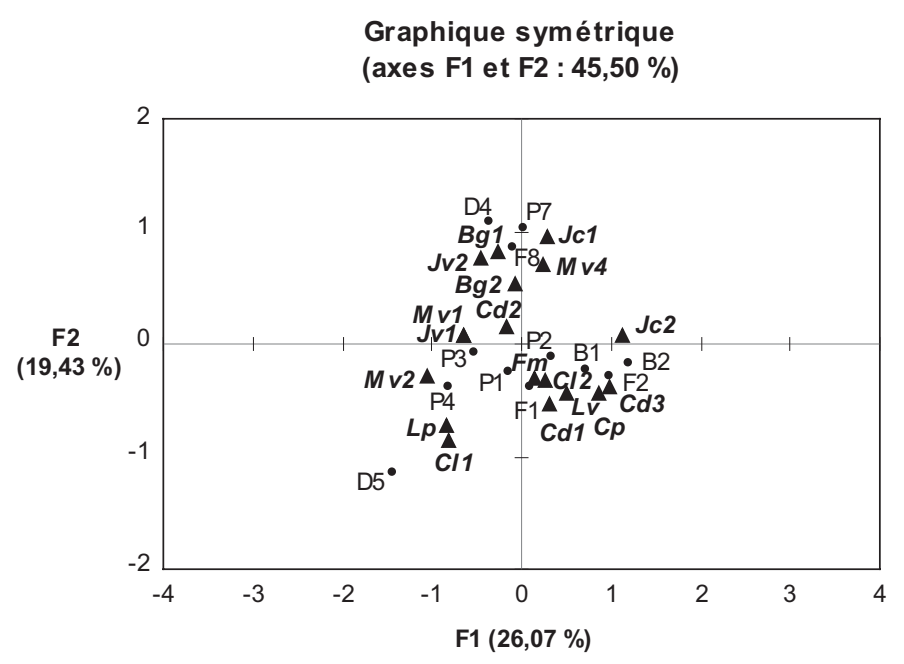

Fig. 7 - AFC des seules séries attribuables au Chasséen ancien.

Correspondence analysis of the early chassean series.

Il est à noter que les anses en ruban/boudin (P1) et les boutons prismatiques ( $\mathrm{P} 2)$, situés près de l'intersection des axes, concernent les trois classes de manière équivalente.

La position excentrée de la classe 4 repose essentiellement sur la présence des décors de cordons larges (D5) qui, hérités du Néolithique ancien et du Néolithique moyen I, peuvent permettre de supposer une chronologie haute pour les séries concernées. Cependant, cet argument unique demeure fragile d'autant que les ensembles de la classe 4 sont restreints.

La séparation entre les classes 1 et 2 est structurée par l'opposition entre les récipients carénés, les multitubulures (P7) et les décors rayés/quadrillés sur assiettes à marli d'une part (classe 2) et les bourrelet/méplats pré-oraux internes sur coupes et bandeaux pré-oraux externes sur jarres de l'autre (classe 1). Ces oppositions non strictes sont suffisamment significatives pour fonder une interprétation cohérente. Les carènes connaissent évidemment un grand succès dans le Chasséen, mais les formes qui leur sont associées changent radicalement dans les phases récentes, tout au moins en Languedoc oriental et en Provence ${ }^{4}$ (Lepère, 2010). Les coupes en calotte de la classe 1, munies d'un bourrelet ou bandeau pré-oral interne, ont un avenir au moins dans la/les première(s) phase(s) du Chasséen récent, et ce dans tout le Midi (François, 2002 ; Gandelin, 2010 ; Lepère, 2010). Faut-il alors envisager un désintérêt temporaire pour les carènes durant le Chasséen ancien, si l'on suppose la succession entre classe 2 et classe 1 ? En outre, le facteur géographique a sans doute une part de responsabilité dans la formation de ces deux classes. En effet, les deux gisements situés en dehors de la zone d'étude (Le Champ du Poste à Carcassonne dans l'Aude et Les Bagnoles à l'Islesur-La-Sorgue dans le Vaucluse) se positionnent dans deux groupes différents de la $\mathrm{CAH}$ (classes 1 et 2) ; ils représentent sans doute bien 2 faciès distincts.

4. Dans l'Aude et le Toulousain, des formes plus ou moins semblables ressurgissent dans les phases les plus récentes du Chasséen (Vaquer, 1990 ; 1991 ; François, 2002 ; Gandelin, 2010).

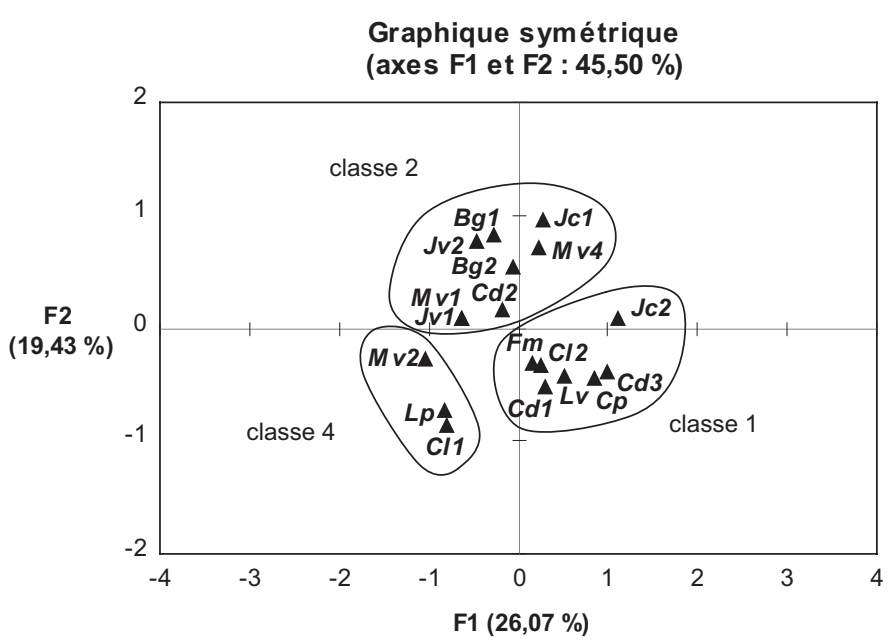

Fig. 8 - AFC des séries anciennes avec les classes données par la $\mathrm{CAH}$.

Classes of the cluster analysis to the graph.

\section{LES STYLES CÉRAMIQUES DU CHASSÉEN EN LANGUEDOC ORIENTAL}

\section{LE STYLE 1}

Ce style (classe 4 de la $\mathrm{CAH}$ ) est représenté sur le site gardois du Mas de Vignole 4 (F8013) et sur les sites héraultais de La Clau (F1062) et de Port Ariane (fig. 9 et 10).

Les éléments les plus caractéristiques sont : des coupes à paroi ouverte munies de tubulures horizontales; des marmites globuleuses ou à inflexion basse munies de cordons multiforés, de tubulures horizontales ou d'anses en ruban; des bouteilles à col sinueux et anses en ruban ; des décors de cordons lisses larges, et plus rarement, de cordons impressionnés et de cannelures jointives. Les récipients carénés semblent absents. Les coupes à marli et à socle ne sont représentées que par un exemplaire au Mas de Vignole 4, F8013. Les jarres à bandeau externe sur le bord de même que les coupes à bourrelet ou bandeau interne sur le bord semblent absentes. Les éléments de préhension sont dominés par les anses en ruban ; cette abondance doit être pondérée par le fait qu'elles ne semblent jamais utilisées comme éléments uniques mais plutôt doubles. Parmi les décors se remarque la composition à base de triangles résillés incisés sur le fragment d'assiette à socle au Mas de Vignole.

$\mathrm{Au}$ regard de ces caractéristiques, on peut ajouter, aux ensembles sériés, plusieurs séries restreintes de La Clau (F1044, F1120 et F1122, notamment), ainsi que le secteur 1 de Saint Antoine à Saint-Aunès dans l'Hérault (Furestier et al., sous presse).

Du point de vue technique, on remarque la présence de récipients à paroi très épaisse. Les pâtes sont marquées par la présence des grosses inclusions mates de calcite, malgré la finesse des parois des récipients dans lesquelles on les trouve souvent. Ce type de pâte concerne encore, à La Clau, plus de la moitié des tessons ; il a également été décrit à Saint Antoine pour le secteur 1 (Furestier et al., 2012). 


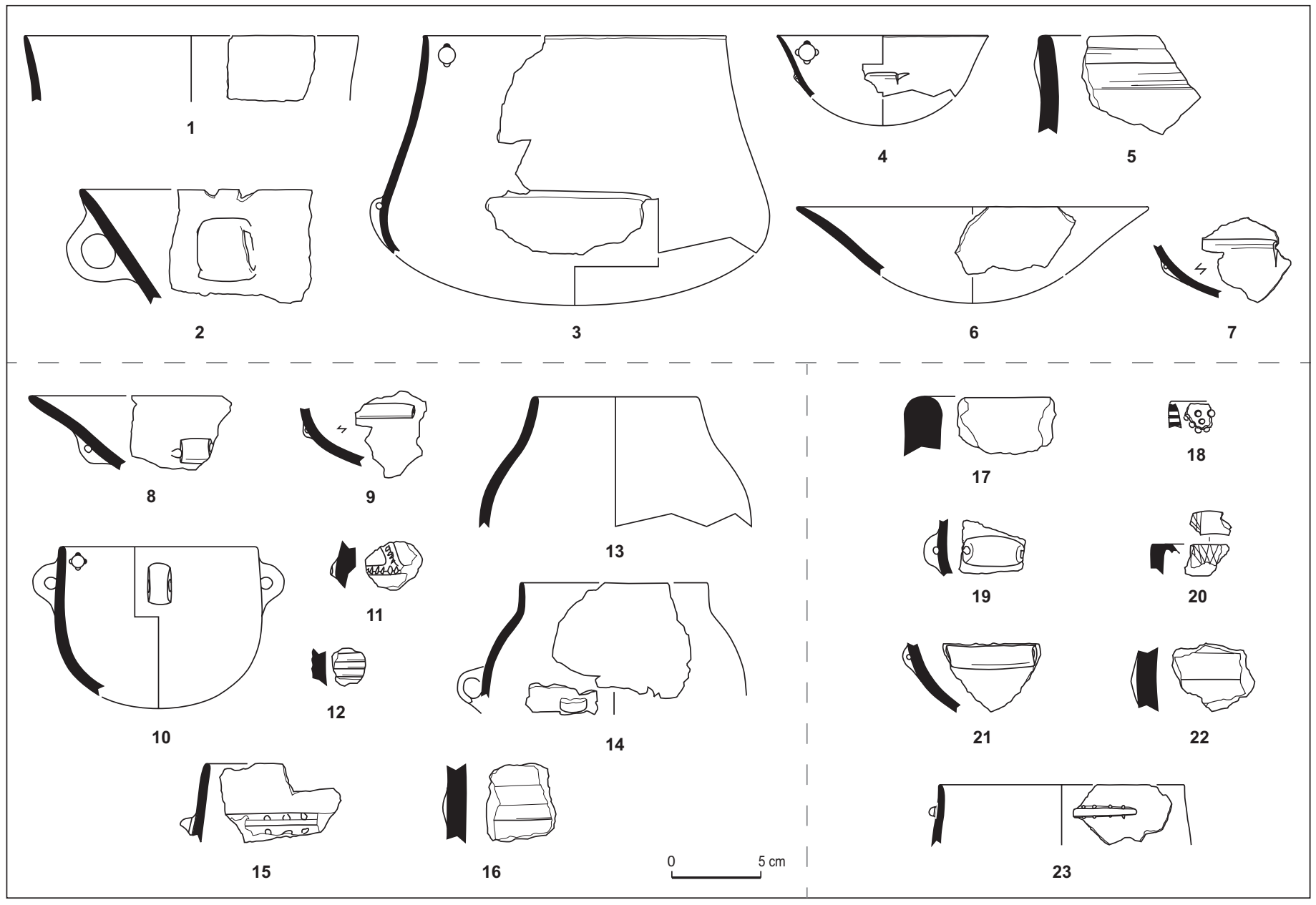

Fig. 9 - Les ensembles composant le style 1 du Chasséen en Languedoc oriental. 34 - Gigean - La Clau III, F1062 (Cl1 : $n^{\circ} 1$ à 7$)$; 34 - Lattes - Port Ariane III, nappes inférieure (21078) et supérieure (21077) de mobilier et structures scellées par ces nappes (Lp : $n^{\circ} 8$ à 16) ; 30 - Nîmes - Mas de Vignole IV, F8013 (Mv2 : n 17 à 23) (Dessins : C. Georjon, INRAP).

Sets for chassean style 1 of Eastern Languedoc. 34 - Gigean - La Clau III, F1062 (Cl1: $n^{\circ} 1$ to 7); 34 - Lattes - Port Ariane III, lower layer (21078) and top layer (21077) and structures associated (Lp: $n^{\circ} 8$ to 16); 30 - Nîmes - Mas de Vignole IV, F8013 (Mv2: $n^{\circ} 17$ to 23) (Drawing: C. Georjon, INRAP).

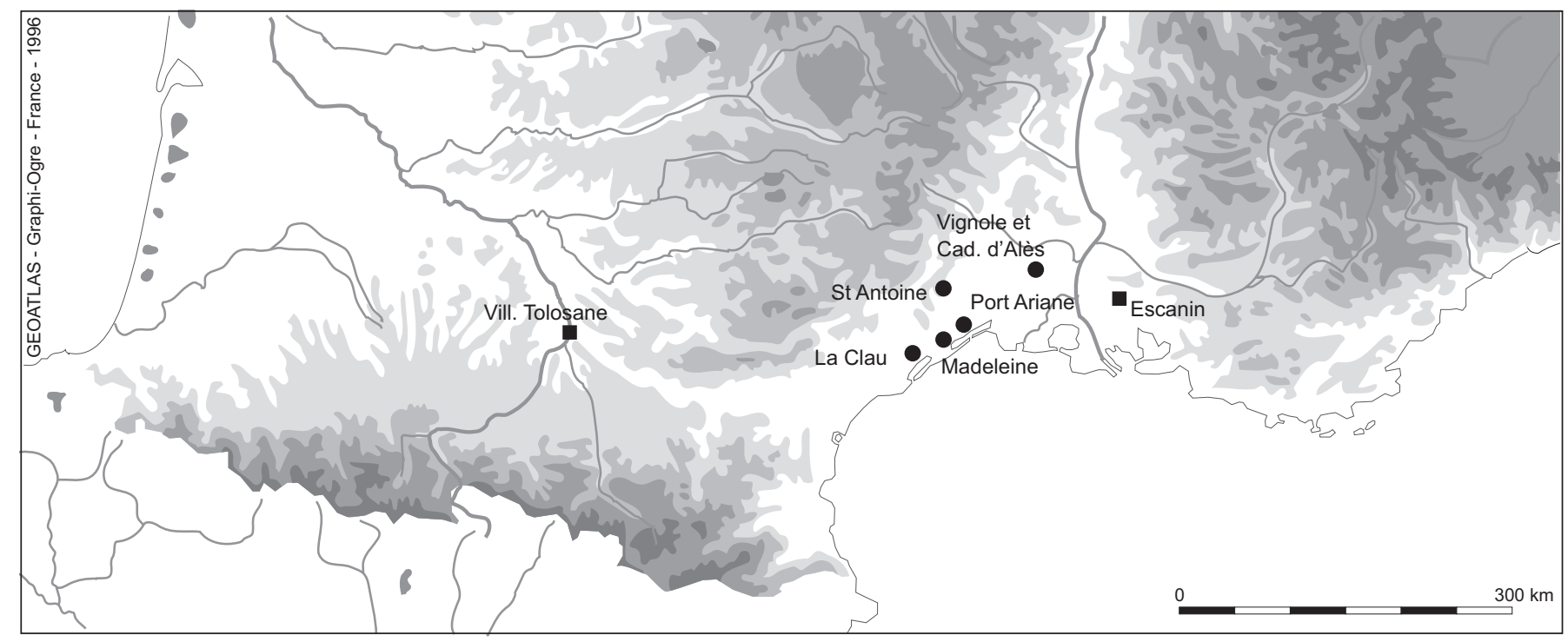

Fig. 10 - Cartographie du style 1 en Languedoc oriental (ronds) et comparaisons en Languedoc occidental et en Provence (carrés).

Sites for chassean style 1 of Eastern Languedoc (circles) and comparisons in Western Languedoc and Provence (squares). 
Ces caractères nous ont incitées à considérer ce style comme une phase initiale du Chasséen. Cependant, d'autres éléments courants dans les étapes anciennes de cette culture sont manquants (multitubulures, jarres à bandeau pré-oral externe, coupes à bourrelet ou bandeau pré-oral interne, récipients carénés...). Le faible nombre des ensembles concernés, leur indigence et parfois leur mauvaise conservation (Port Ariane) nous incite à la prudence concernant la réalité de ce style que l'on pourrait considérer comme un Proto-Chasséen, c'est-à-dire comme un Chasséen incomplet.

Nous ne voyons que deux séries de comparaison à ce style 1 ; il s'agit de E52 à Villeneuve-Tolosane dans le Lot-etGaronne (François, 2002) et des couche 3 à 6 selon les secteurs d'Escanin aux Baux-de-Provence dans les Bouches-du-Rhône (Lepère, 2002). Ces affinités se fondent sur des convergences générales (sobriété de ces ensembles, absence de carènes), et sur quelques éléments précis (cordons et impressions à Escanin). On n'y retrouve pas les premiers caractères proprement chasséens (coupes à marli ou à socle, décor rayé quadrillé, préhensions multiforées) qui sont présents dans le style 1.

\section{LE STYLE 2}

Les classes 1 et 2 de la CAH montrent un enrichissement important du corpus avec notamment 3 types de formes clés : le bol ou la marmite à carène basse et paroi haute, verticale ou fermée ; l'assiette ou la coupe à bourrelet ou méplat pré-oral interne ; la jarre à bandeau pré-oral externe. La différence entre les classes 1 et 2 se fait avant tout sur un dosage de ces types de formes, c'est la raison pour laquelle nous considérons que les classes 1 et 2 sont des variantes d'un même style que nous nommerons $2 \mathrm{a}$ (classe 2 ) et $2 \mathrm{~b}$ (classe 1 ).

La variante $2 \mathrm{a}$ (fig. 11) rassemble les séries gardoises de F2123 au Mas de Vignole 4, F1128 au Mas de Vignole 11, F3162 au Cadereau d'Alès, et héraultaises de F1068 et F1095 des Jardins de Vert Parc, F22 de Jacques Cœur 2, ainsi que les séries vauclusiennes de comparaison, F48 et F75 des Bagnoles. D'autres ensembles plus restreints s'ajoutent à cette liste : F1129 du Mas de Vignole 11, F1084, US1077, F1016, F1044, F1085 des Jardins de Vert Parc.

Cette variante du style 2 est marquée par la forte présence des bols et marmites à carène basse et paroi haute. Elles sont accompagnées de formes similaires dont la rupture de pente n'est pas une carène mais une inflexion. Ces récipients sont sans préhensions ou bien munis d'une tubulure horizontale sur la segmentation, visiblement comme mono-anse. Quelques profils sinueux sont présents. Les bouteilles sont à col sinueux. Les bourrelets ou méplats pré-oraux internes sur les assiettes simples sont sporadiquement présents.

Du côté des préhensions, les anses en ruban sont toujours nombreuses, accompagnées de tunnelliformes et de cordons multiforés. Les multitubulures alternant avec des bandeaux plats apparaissent. Le décor rayé/quadrillé est plus fréquent et s'applique au marli des assiettes. Les chevrons cannelés sur le méplat alternant avec les multitubulures du récipient de Jacques Cœur demeure isolé. Notons enfin que les pâtes de ces séries ne contiennent plus que rarement des inclusions mates et grossières.

La variante $2 \mathrm{~b}$ (fig. 12) est présente au Lavous couche IIb fosse, au Cadereau d'Alès, F1094 et F5129 et à Fumérian, F1092, dans le Gard, à La Clau, F1195, dans l'Hérault. La situation de F9 de jacques Coeur 2 sur le plan factoriel, quoique cet ensemble apparaisse dans la classe 1 de la CAH, est intermédaire entre les variantes 2 a et $2 b$. Il ne peut-êre intégré sans difficulté dans l'une ou l'autre des deux variantes et paraît représenter un faciès mixte. Des ensembles plus restreints peuvent lui être raccordés à la variante $2 b$, comme F062 de La Capoulière 2 à Mauguio dans l'Hérault, la couche VII de la grotte de La Madeleine (inédit), ainsi que plusieurs ensembles céramiques de Saint Antoine (Furestier et al., sous presse).

Cette variante est caractérisée par les assiettes et coupes à bourrelet ou méplat pré-oral interne et par les jarres à bandeau pré-oral externe. Les carénés, du même type que ceux du style 2 sont présents mais rares. Les marmites à inflexion basse et paroi haute semblent un peu mieux représentées. Les types de préhension sont les mêmes qu'en 2 a à l'exception notable des multitubulures, absentes ici. Les décors introduisent, outre le décor rayé/quadrillé sur les assiettes à marli et à socle, dont un exemplaire recto verso à La Madeleine, des demi-cercles emboités incisés au-dessus des ruptures de pente (Cadereau d'Alès et Jacques Cœur). Enfin, comme pour le style 2a, les pâtes à inclusions mates et grossières sont rares.

En dehors du Languedoc oriental, les comparaisons pour le style 2 montrent que les variantes que nous avons relevées ne sont pas toujours distinctes (fig. 13).

Dans certains ensembles antérieurs au Chasséen ancien, des coupes et marmites carénées mono-ansées à col cylindrique ou tronconique, évoquant plus ou moins celles de notre style $2 \mathrm{a}$, sont déjà présentes et couvrent un large territoire (VilleneuveTolosane dans le Lot-et-Garonne, E52; Font Juvénal à Conques dans l'Aude, couche 11, baume de Fontbrégoua à Salernes dans le Var, couches 38-30, Saint-Uze de la grotte du Gardon à Ambérieu-en-Bugey dans l'Ain, Pontcharaud 2 à ClermontFerrand et La Pradelle à Pont-du-Château dans le Puy-de-Dôme ; François, 2007 ; Guilaine et al., 1990 ; Luzi et Courtin, 2001 ; Nicod, 2009 ; Loison et Gisclon, 1991 ; Jallet et Georjon, sous presse). Bien sûr, les éléments qui accompagnent ces récipients ont généralement peu de points communs avec la variante $2 \mathrm{a}$.

Fig. 11 - les séries composant le style 2 du Chasséen en Languedoc oriental, variante 2a. 30 - Nîmes - Mas Neuf et Cadereau d'Alès, F3162 (Cd2: $n^{\circ} 1$ à 6) ; 30 - Nîmes - Mas de Vignole 11, F1128 (Mv4: $n^{\circ} 7$ à 11); 30 - Nîmes - Mas de Vignole 4, F2123 (Mv1: $n^{\circ} 12$ et 13); 34 - Montpellier - Jacques Coeur 2, synthèse de F22 (Jc1: $n^{\circ} 14$ à 18) ; 34 - Castelnau-le-Lez - Les Jardins de Vert Parc - synthèse de F1068 (Jv1 : $n^{\circ} 19$ à 26), F1095 (Jv2: $n^{\circ} 27$ à 31) (Dessins : C. Georjon et S. Lancelot, INRAP).

Sets for chassean style 2 of Eastern Languedoc, 2a variant. 30 - Nîmes - Mas Neuf et Cadereau d'Alès, F3162 (Cd2: $n^{\circ} 1$ to 6); 30 - Nîmes - Mas de Vignole 11, F1128 (Mv4: $n^{\circ} 7$ to 11); 30 - Nîmes - Mas de Vignole 4, F2123 (Mv1; $n^{\circ} 12$ to 13); 34 - Montpellier - Jacques Coeur 2, synthesis of F22 (Jc1: $n^{\circ} 14$ to 18); 34 - Castelnau-le-Lez - Les Jardins de Vert Parc - synthesis of F1068 (Jv1: $n^{\circ} 19$ to 26 ), F1095 (Jv2: $n^{\circ} 27$ to 31) (Drawings: C. Georjon et S. Lancelot, INRAP). 


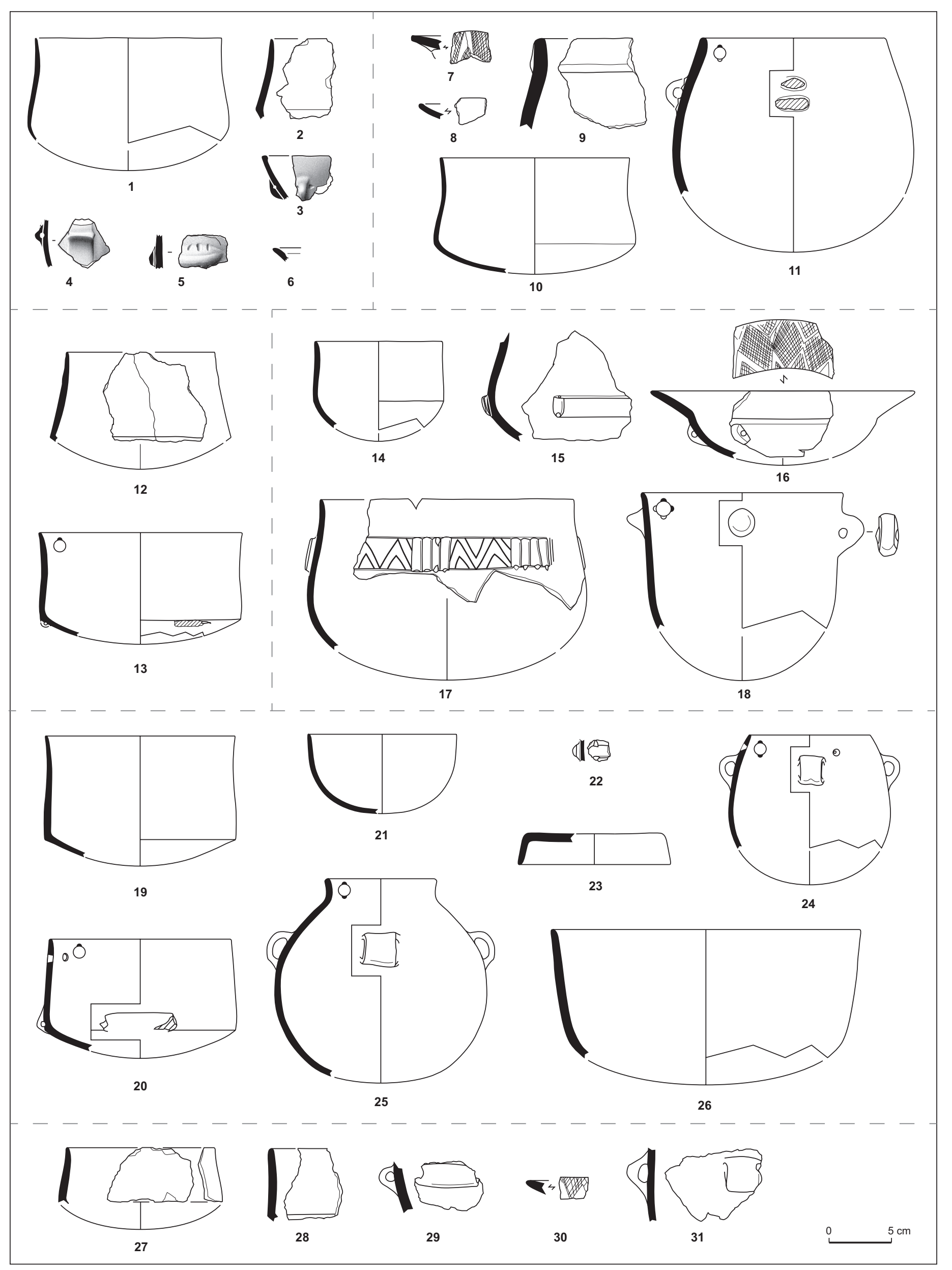




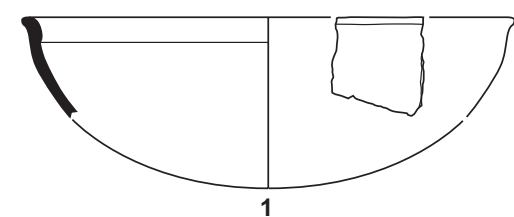

1 Pon
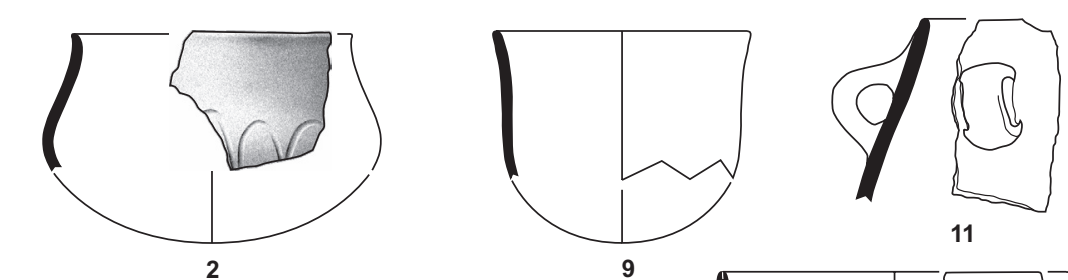

$1 \sqrt{6} \quad 0 \sum_{7}$
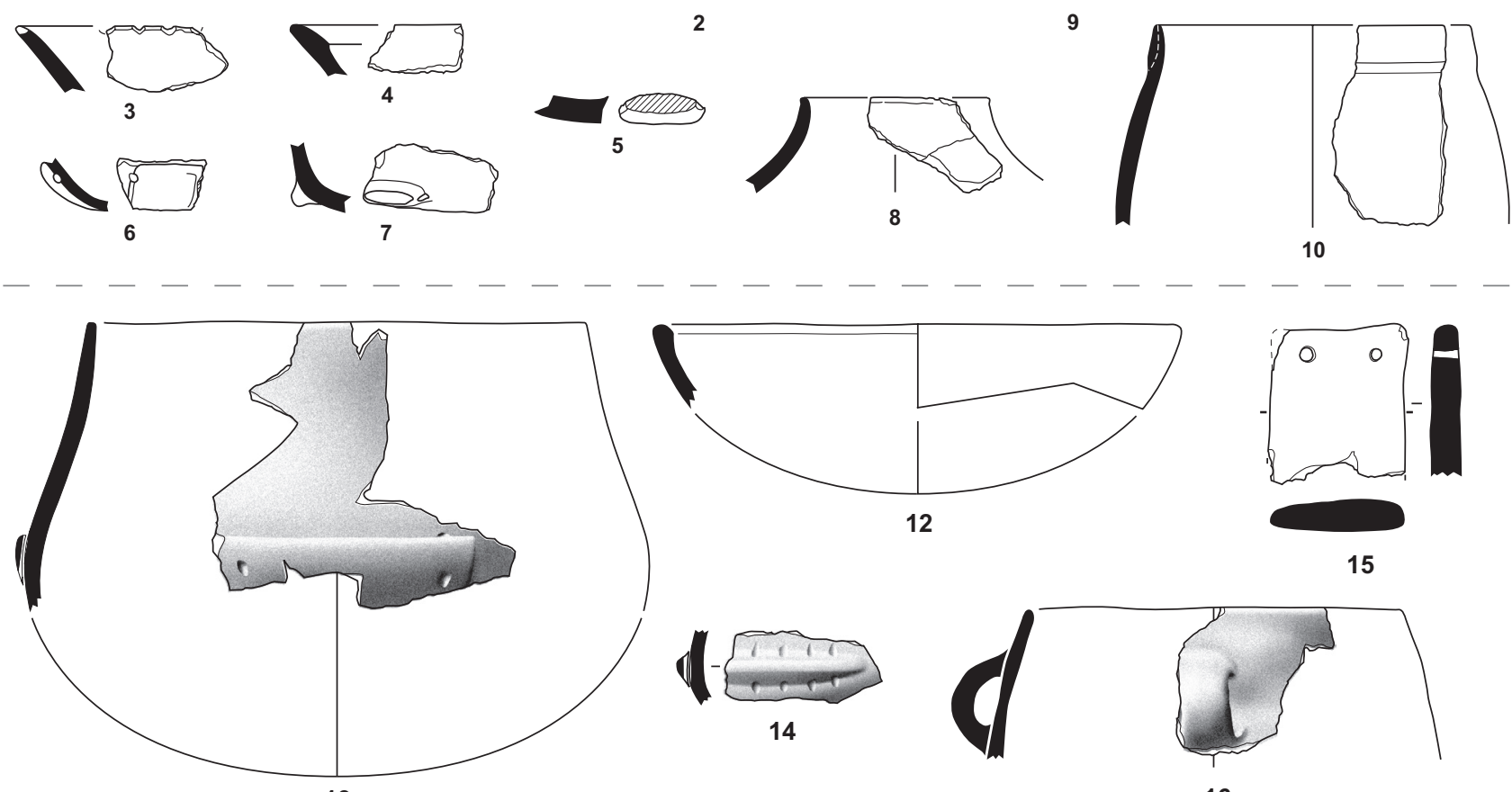

13
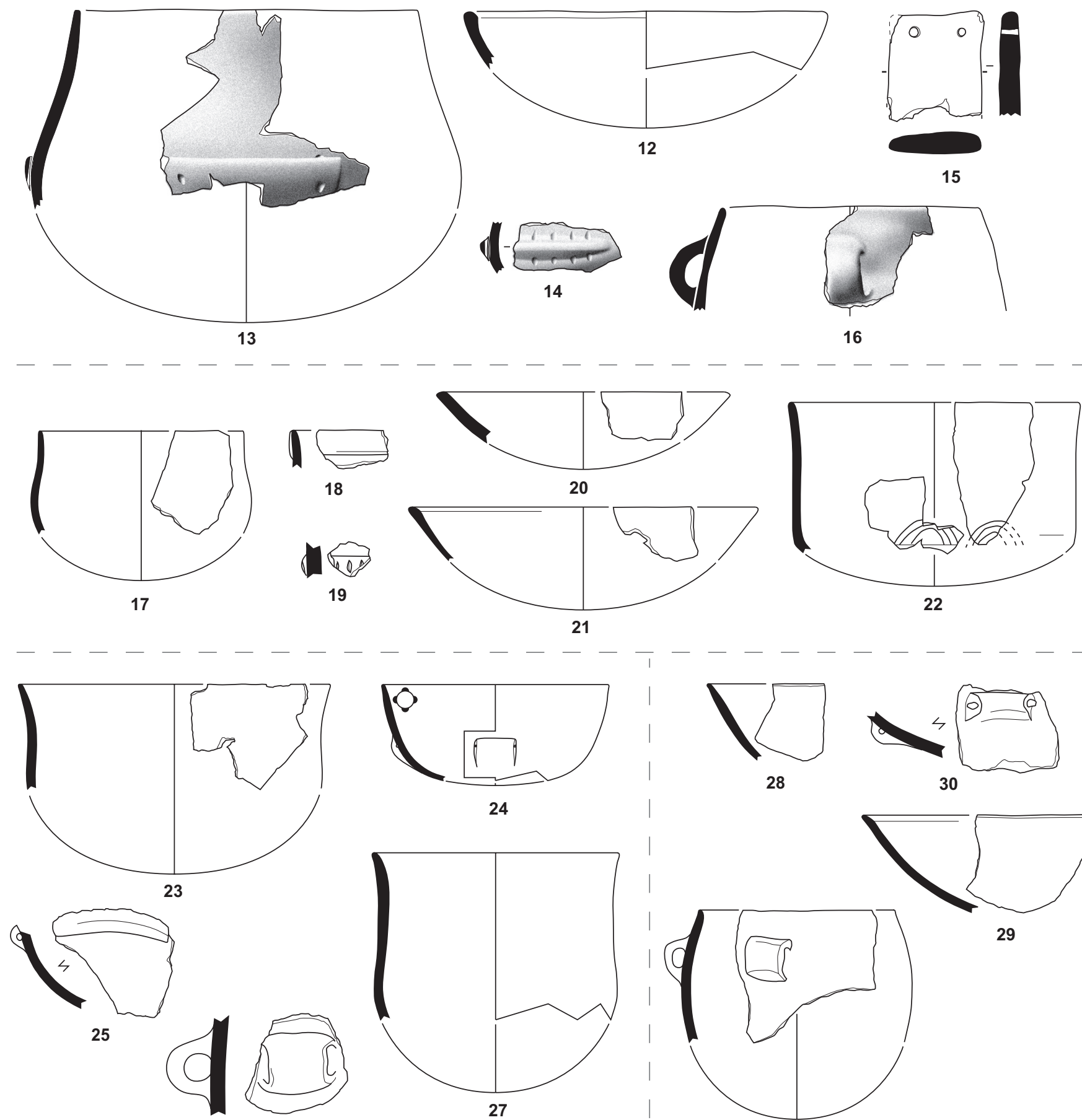

26

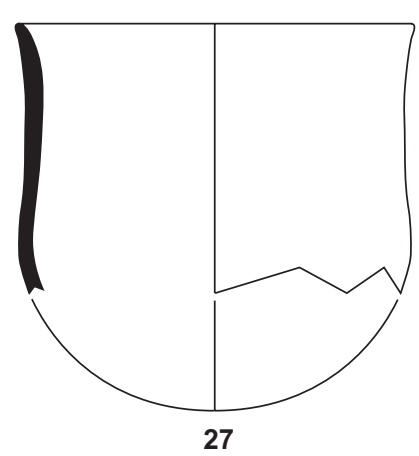

28
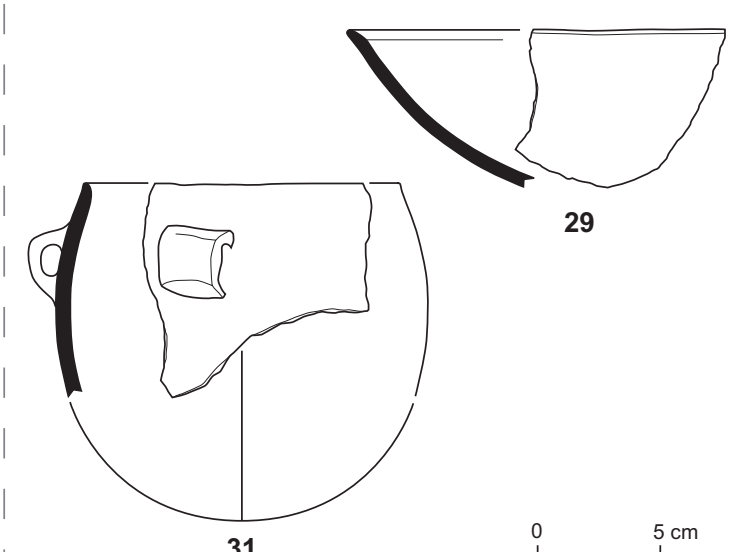

29 


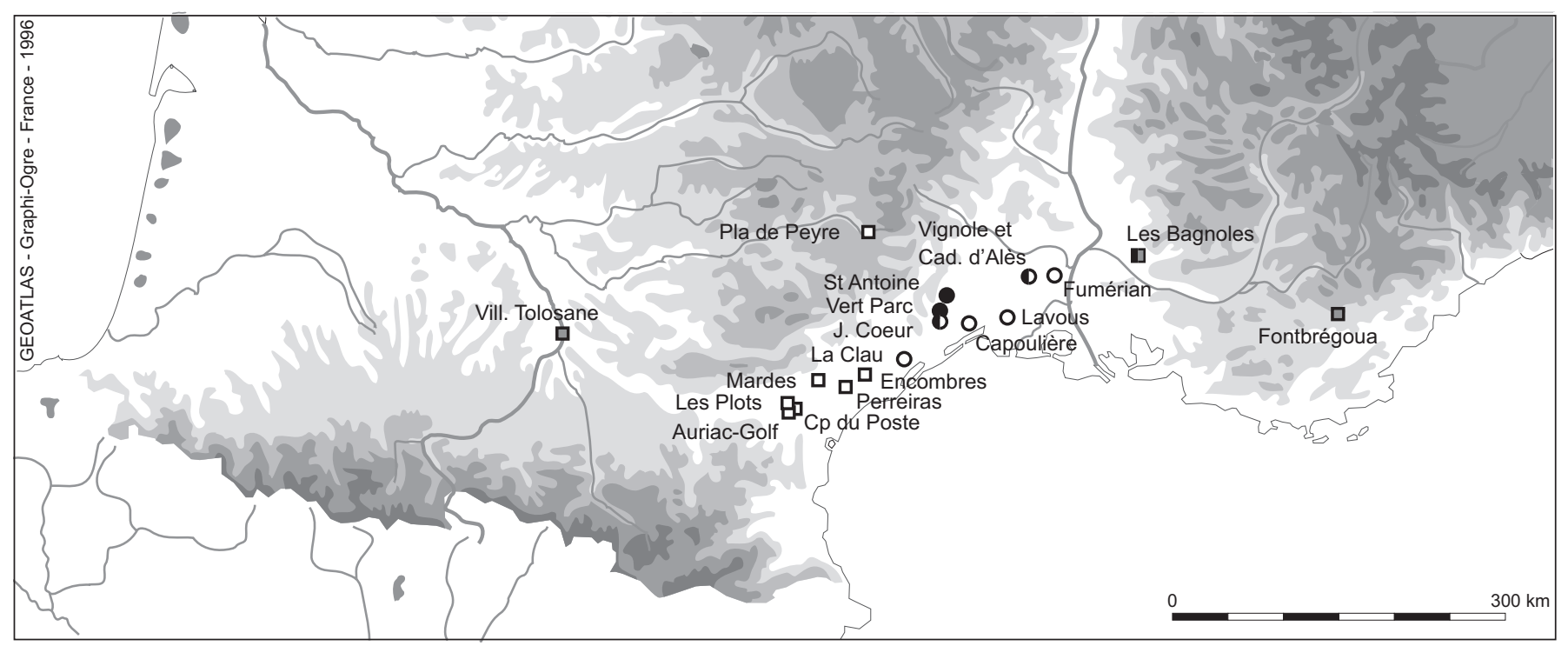

Fig. 13 - Cartographie des sites présentant le style 2 du Chasséen en Languedoc oriental (ronds) et comparaisons hors de la zone d'étude (carrés). En noir : variante $2 a$, en blanc : variante $2 b$, en gris : variante mixte.

Sites for chassean style 2 of Eastern Languedoc (circles) and comparisons outside of the study area (squares). In black: $2 a$ variant, in white: $2 b$ variant, in grey: mixed variant.

Dans le Chasséen ancien de Provence et du Languedoc occidental, ces formes semblent rarement isolées des caractéristiques de la variante $2 \mathrm{~b}$ (coupes à bourrelet ou méplat pré-oral interne et jarres à bandeau pré-oral externe). Seule F48 du site des Bagnoles s'y conforme. F75, pourtant classée en 2a principalement grâce à la présence d'une multitubulure, est mixte, tout comme les ensembles représentatifs du Chasséen ancien à Villeneuve-Tolosane (D4-7, CX18, CX19, CX22, CX23, CX26, CX79 ; François, 2002) ainsi, semble-t-il que les couches 29-25 de la baume de Fontbrégoua, si l'on admet la stricte homogénéité de ces niveaux (Lepère, 2009).

Par contre, les équivalents de la variante $2 \mathrm{~b}$ pure sont nombreux dans la partie occidentale de l'Hérault et dans l'Aude et où ils ont servi de définition au style des Plots (site éponyme à Berriac, Auriac-Golf et le Champ du Poste à Carcassonne, Perreiras à Pouzols, les Mardes à Cesseras, Encombres à Quarante, F1069 ; Vaquer, 1990 ; Gaillard, 2003 ; inédit, étude C. Georjon ; Ambert et al., 1988 ; Amiel et Jédikian, 2003) ; le site quercynois du Pla de Peyre à Creissels dans l'Aveyron est également concerné (Fouéré et al., 2003 ; Gernigon, 2004). On notera enfin qu'en dehors de la Ligurie (Giribaldi, phase C ; Binder, 1984 ; Crepaldi, 2004) et peut-être de la Drôme (Chasséen A ; Beeching, 1995 ; 2002), les formes carénées ouvertes sont très rares voire absentes dans le Chasséen ancien, de la Provence au Garonnais et au Querey.

\section{LE CHASSÉEN STYLE 3}

Ce style correspond à la classe 3 de la CAH. Il concerne la grotte de la Madeleine (fouille L. Barral), couche VI-I, Mas de Vignole 4, F8056, ainsi que le Cadereau d'Alès, F1043, F1063 et F1055. Il est également présent dans plusieurs ensembles de Saint Antoine (Furestier et al., 2012).

Bien que l'AFC n'ait pas permis de le relever, il y a selon nous une légère évolution dans ce style qui fait ressortir une variante $3 a$ et une variante $3 b$ (fig. 14 et 15 ).

La variante $3 \mathrm{a}$ est représentée par La Madeleine couches VI à IV, Mas de Vignole 4 F8056 et Cadereau d'Alès F1043, F1055. Elle se caractérise par la perduration de caractères anciens (anses en ruban, coupes à bourrelet ou bandeau pré-oral interne, jarres à bandeau pré-oral externe, marmites à inflexion basse, bouteilles à col sinueux, coupes à marli ou à socle ornées) et par une nouvelle diversification des formes, qui voit l'apparition des ressauts et des épaulements. Les formes carénées les plus courantes sont des coupes à segmentation basse à col demi-haut ouvert mais sont également présentes des coupes carénées étroites à segmentation basse et col demi-haut fermé. Les coupes à carène haute et assiettes carénées font une apparition discrète. Les marmites à épaulement et les bouteilles à col anguleux sont fréquentes. Les moyens de préhension et suspension se renouvellent également avec l'apparition

4 Fig. 12 - Les séries composant le style 2 du Chasséen en Languedoc oriental, variante $2 b .30$ - Nîmes - Mas Neuf et Cadereau d'Alès, F5129 (Cd3 : $n^{\circ} 1$ à 11), F1094 (Cd1 : $n^{\circ} 12$ à 16) ; 34 - Montpellier - Jacques Coeur 2, F9 (JC2 : $n^{\circ} 17$ à 22) ; 34 - Gigean - La Clau III, F1195 (Cl2 : $n^{\circ} 23$ à 27) ; 30 - Manduel - Fumérian, F1092 (Fm : $n^{\circ} 28$ à 31) (Dessins : C. Georjon et S. Lancelot, INRAP).

Sets for chassean style 2 of Eastern Languedoc, $2 b$ variant. 30 - Nîmes - Mas Neuf and Cadereau d'Alès, F5129 (Cd3: $n^{\circ} 1$ to 11), F1094 (Cd1: $n^{\circ}$ 12 to 16); 34 - Montpellier - Jacques Coeur 2, F9 (JC2: $n^{\circ} 17$ to 22); 34 - Gigean - La Clau III, F1195 (Cl2: $n^{\circ} 23$ to 27); 30 - Manduel - Fumérian, F1092 (Fm: $n^{\circ} 28$ to 31) (Drawing: C. Georjon et S. Lancelot, INRAP). 


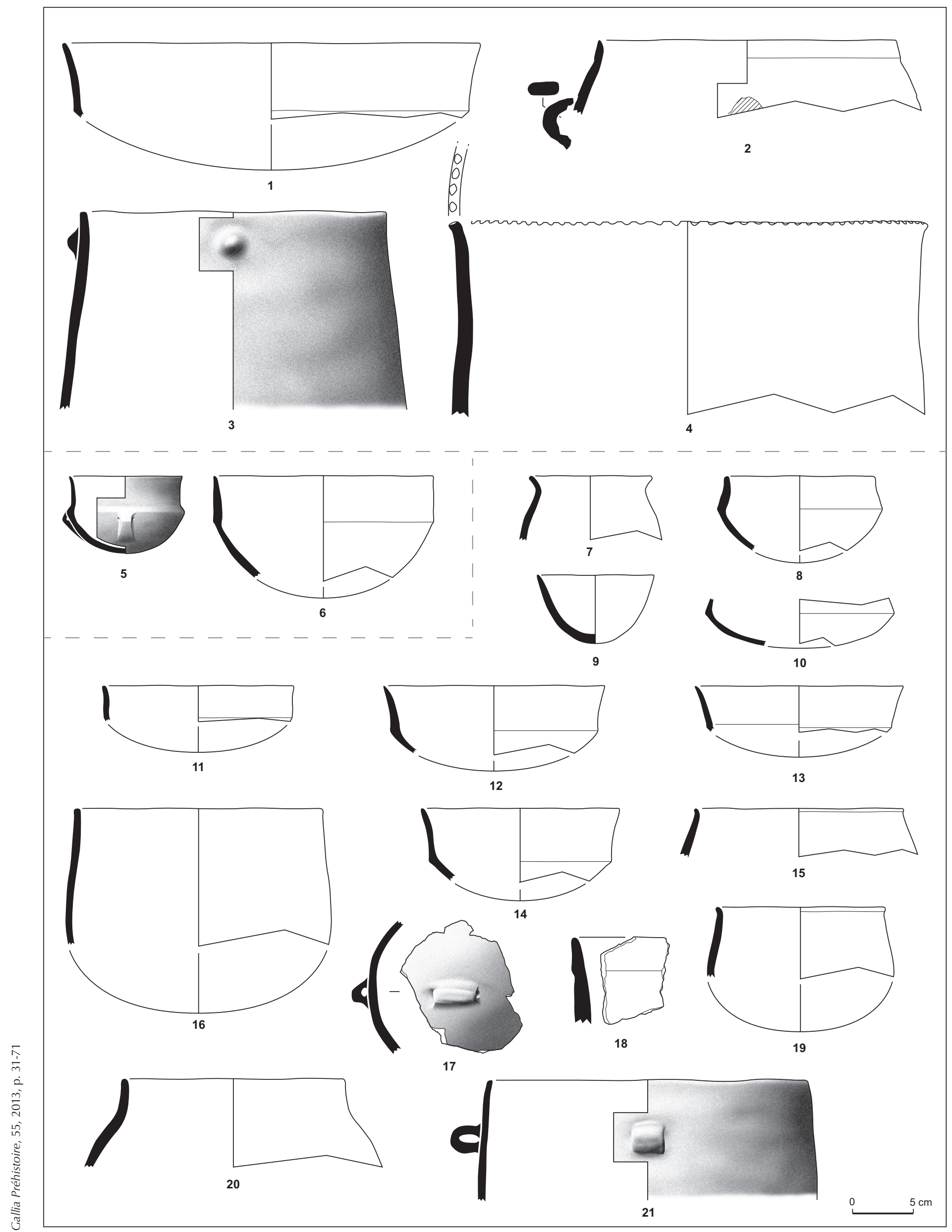


des plaquettes multiforées ou multitubulées. Parmi les décors, ce sont les moustaches incisées sur coupes simples ou segmentées et les perforations traversières périphériques sous le bord de coupelles qui créent la nouveauté. Les sillons internes uniques sur coupes, très rarement attestés dans le style 2, prennent ici leur essor.

Dans la variante 3 b qui isole les couches IV à I de La Madeleine et F1063 du Cadereau d'Alès, les archaïsmes ont presque totalement disparu et la diversification des formes segmentées s'accentue (présence de pots à ressaut ou à épaulement, diversification des types d'épaulement). La présence d'assiettes carénées préfigure le style 4.

Bien que le style 3 réunisse le plus grand nombre de caractères propres au Chasséen, il est difficile d'établir des comparaisons hors de notre zone d'étude (fig. 16). En Languedoc occidental et en Quercy, les niveaux B et A inférieur de Capdenac-le-Haut dans le Lot (Gernigon, 2004), de même que les séries attribuées au Chasséen récent du groupe $3 \mathrm{a}$ de Villeneuve-Tolosane (François, 2007) et la fosse F1 des Monges à Launaguet en Haute-Garonne (Servelle, 1980) montrent des points communs avec le style $3 \mathrm{a}$ du Languedoc oriental : perdurations anciennes (coupes à bourrelet ou bandeau pré-oral interne, jarres à bandeau pré-oral externe, anses en ruban), majorité de carénés bas et ouverts, décors de moustaches. Cependant, on ne retrouve ni dans le Toulousain, ni dans le Quercy, les bols et marmites à épaulement. D'autres différences flagrantes avec le Languedoc oriental laissent entendre que ces ensembles occidentaux appartiennent à un autre faciès.

En Provence, le style 3a correspond, semble-t-il, à l'étape B de C. Lepère (2012). Caucade à Nice dans les Alpes-Maritimes et les couches 9-7 de la grotte de l'Église Supérieure à Baudinard dans le Var présentent également l'association entre éléments anciens et formes évoluées (Lepère, 2009 ; 2012). La distinction entre les variantes $3 a$ et $3 b$ est à mettre en parallèle avec le phasage du puits R21-1 de VilleneuveTolosane (Jédikian, 1999 ; Gandelin, 2011) qui se fonde sur la perduration d'éléments archaïsants (niv. 13-8) et sur leur quasidisparition ensuite (niv. 7-1). La variante 3b pourrait de même être mise en parallèle avec l'étape $\mathrm{C} 1$ du Chasséen provençal où l'on trouve Fontbrégoua couches 24-19, notamment. Les perdurations se sont estompées et les décors sur coupes simples ainsi que les bols et marmites à ressaut sont plus nombreux. Cependant, les sites provençaux montrent des divergences par rapport au style 3, particulièrement la phase $\mathrm{C} 1$ qui intègre des assiettes à marli incliné ou à bord déversé ornés notamment, et ne s'inscrivent donc pas dans le même faciès.

\section{LE CHASSÉEN STYLE 4}

La classe 6 de la CAH donnant le style 4 est représentée par deux ensembles de faible ampleur, La Ramière F5084 et 8048 et par la série plus étoffée de Plaine de Chrétiens F39 (fig. 17).
On peut y adjoindre les séries de Mas d'Embec à Saint-Pargoire (Briois, 1998), la série issue des prospections de Saint Jean de Nozé à Lunel (Garrido, 1990) et une grande partie du mobilier de Filliès I (Port Antique) de Lattes dans l'Hérault (Mendoza et Prades, 1988).

Cette association est caractérisée par le développement des assiettes carénées aux côtés des coupes à carène basse et paroi demi-haute. Les coupes à carène haute demeurent rares. Les bols et marmites à épaulement sont présents ; ils sont accompagnés par les premiers gobelets épaulés à col haut. Les bandeaux multiforés apparaissent ainsi que les doubles perforations intra-pariétales sur la carène. Les décors de sillons internes connaissent un important développement. Ces motifs sont uniques ou multiples et parfois accompagnés de décors internes complémentaires. Un fragment de couvercle à décor de type Lagozza et une préhension interne sont présents à La Ramière.

Le style 4 est comparable (fig. 18) avec celui d'Auriac qui se rapporte au site éponyme, situé à Carcassonne (Aude ; Guilaine et Vaquer, 1973 ; Vaquer, 1990 ; 1991 ; Jédikian, 2000) mais aussi avec la phase C2 de Provence (Lepère, 2012). Les principales caractéristiques de ces ensembles (assiettes carénées, carènes perforées, sillons internes multiples avec ou sans décors complémentaires, couvercles à décor de type Lagozza) ont une large répartition, depuis le pied des Pyrénées (Mas Coudine au Boulou dans les Pyrénées Orientales ; Vignaud, 1993) jusqu'à la Provence orientale (Les Terres Longues à Trets dans les Bouches-du-Rhône, notamment ; Lepère, 2009 ; 2012 ; Léa et al. 2010 ; Léa, 2012) en passant par la vallée du Rhône (Les Moulins à Saint-Paul-Trois-Châteaux, F131 ; L'Héritière II à Vernègues, F10 ; Beeching, 2002 ; Chapon et al., 2002).

En garonnais et en Quercy, ce style correspondrait avec le Chasséen récent 1 de Villeneuve Tolosane (François, 2007 ; Gandelin, 2011) et le niv. A, horizons d, e, f de Capdenac-leHaut (Gernigon, 2004). Sur ces sites, les assiettes carénées sont concurrencées par des coupes à carène basse et paroi haute ouverte ; les profils à ressaut ou épaulement et les décors internes sur les assiettes, autres que les sillons uniques ou doubles, sont rares.

Le style d'Auriac paraît demeurer au contact de la plaine littorale et de la basse vallée du Rhône ; dès qu'on s'en éloigne les variations stylistiques deviennent plus accusées.

\section{LE CHASSÉEN STYLE 5}

A la classe 5 de la CAH correspond le style 5 . Il est représenté par les séries de La Roquette, F1020 des Jardins de Vert Parc, F4094 du Mas de Vignole 4 et l'ensemble vauclusien de Rocalibert (fig. 19). D'autres ensembles gardois peu étoffés pourraient également s'y rattacher (Le Damier à Nîmes, La Roquette à Tresques, notamment; inédit, fouille J.-Y. Breuil et M.-L. Hervé ; Georjon, 2000).

Le style 5 se caractérise par deux principales catégories

4 Fig. 14 - Les ensembles composant le style 3 du Chasséen en Languedoc oriental. Variante $3 a$ : 30 - Nîmes - Mas Neuf et Cadereau d'Alès, F1043 (Cd5: $n^{\circ} 1$ à 4), F1055 (Cd4 : $n^{\circ} 7$ à 21); variante 3b:F1063 (Cd6: $n^{\circ} 5$ et 6) (Dessins et mise au net : S. Lancelot, INRAP).

Sets for chassean style 3 of Eastern Languedoc, 3a variant: 30 - Nîmes - Mas Neuf and Cadereau d'Alès, F1043 (Cd5: $n^{\circ} 1$ to 4 ), F1055 (Cd4: $n^{\circ} 7$ to 21); $3 b$ variant: F1063 (Cd6: $n^{\circ} 5$ to 6) (Drawing: S. Lancelot, INRAP). 


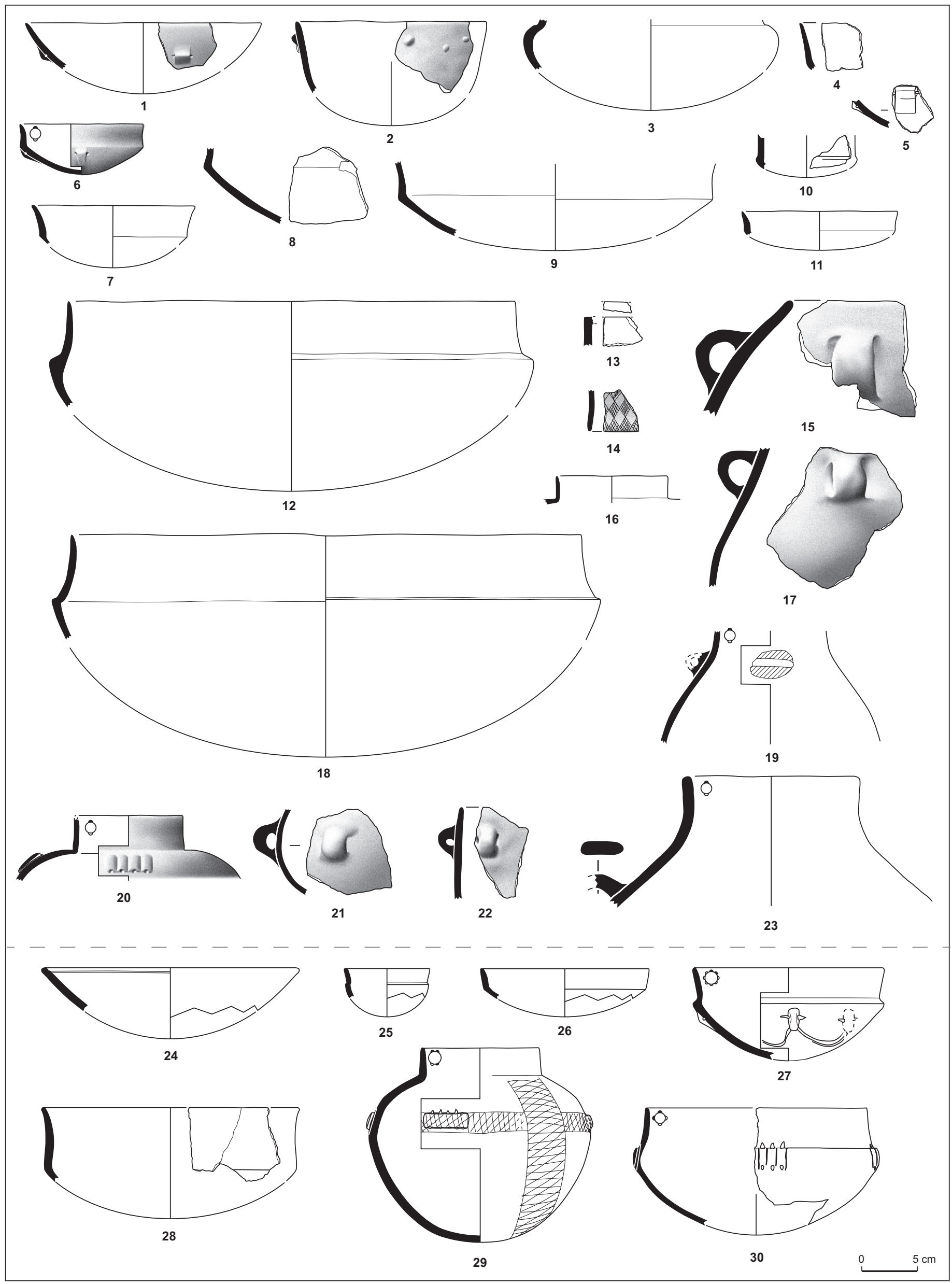




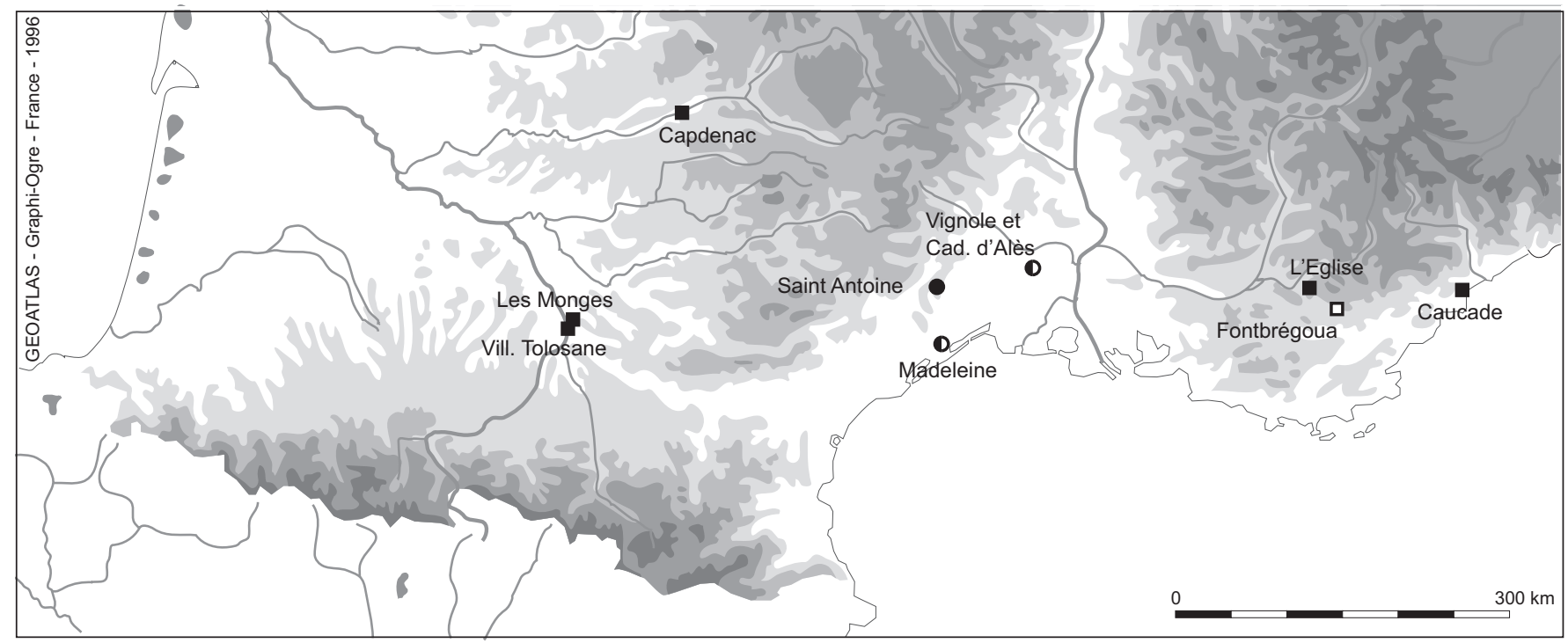

Fig. 16 - Cartographie du style 3 du Chasséen en Languedoc oriental (ronds) et sites de comparaison (carrés). En noir : variante 3a et sites hors zone d'étude présumés contemporains; en blanc : variante $3 b$ et sites hors zone d'étude présumés contemporains.

Sites for chassean style 3 of Eastern Languedoc (circles) and comparisons (squares). In black: 3a variant and contemporaneous sites outside of the study area; in white: $3 b$ variant and contemporaneous sites outside of the study area.

de formes basses segmentées : des coupes surbaissées à col déversé et des coupes carénées ou infléchies à col très court et un fond bien bombé. Certains de ces récipients présentent un renflement interne du col (cran). Les bords ourlés, sur des coupes simples ou carénées et des marmites, sont fréquents (Jardins de Vert Parc, La Roquette de Cavillargues, Rocalibert). Les récipients à épaulement sont de formes diverses ; parmi eux, les gobelets et jarres à épaulement bas et haut col se multiplient. Les préhensions les plus fréquentes sont des rubans ou de longs cordons saillants multiforés. Les décors sont rares. Les sillons internes, généralement uniques, sont peu présents ; un décor sur la panse d'une bouteille est à noter (Vert Parc).

Une variante de ce style pourrait être représentée par une bonne partie des vestiges du site de Raffègues à Mèze dans l'Hérault (Montjardin et Rouquette, 1988) et par les séries des grottes gardoises (fig. 20), issues de collectes sélectives anciennes (grotte des Fées à Tharaux, grotte de Saint Vérédème à Sanilhac-et-Sagriès, grotte du Taï à Remoulins, de la Citerne à Méjane-le-Clap etc., Louis, 1953 ; Vaquer, 1975 ; Timsit, 1994). Les carénés à segmentation basse, notamment ceux « à cran » interne, paraissent plus nombreux. Des bouteilles à col marqué par un simple sillon apparaissent. Les décors se multiplient sur plusieurs types de récipients : boutons en ligne disposés contre le bord de bols et de gobelets, boutons uniques ou doubles sur la carène, thèmes incisés très divers disposés sur le fond externe des coupes carénées et sur la panse des bouteilles.

On nommera donc variantes $5 \mathrm{a}$ et $5 \mathrm{~b}$ les deux types d'assemblages supposés.
Le style 5 fait le lien entre le groupe audois de Bize et les styles C et D du Chasséen de la Drôme ; le Toulousain et le Quercy semblent à l'écart de ces manifestations (fig. 21).

La variante 5 a présente une bonne correspondance stylistique avec la phase D1 de C. Lepère (2012) dans laquelle est inclus Rocalibert, ainsi qu'avec La Roberte, F46 à Châteauneufdu-Rhône dans la Drôme (Beeching, 2002). Il semble que ces assemblages soient relativement localisés aux abords de la vallée du Rhône.

La variante $5 \mathrm{~b}$ est plus difficile à appréhender. Une partie de ses éléments montrent des relations avec le Languedoc occidental, une autre partie avec la Provence et la Drôme. Les carénés "à cran » font le lien entre toutes ces régions, quoiqu'en rive gauche du Rhône, leur répartition semble limitée aux départements proches du fleuve (Vaucluse et à la Drôme). Tout comme ces formes, les décors linéaires en creux de méandres et guirlandes à remplissages sont des éléments de définition du groupe de Bize (Petite grotte de Bize, Bram à Buzérens, La Poste Vieille à Pézens... Vaquer, 1990 ; 1991 ; Guilaine, 1997b) qu'on ne retrouve pas au-delà du Languedoc, en rive gauche du Rhône. Les bols, marmites ou gobelets à col haut et carène basse qui entrent également dans la définition du Bizien (id. ibid.), semblent absents du Languedoc oriental. Ces formes se retrouvent dans le style audois de Cavanac, dans le Chasséen 3b de Villeneuve-Tolosane (François, 2007 ; Gandelin, 2011) et dans le niv. A, horizon a, b, c de Capdenacle-Haut (Gernigon, 2004) dont les autres composantes n'ont pas grand-chose à voir avec le Bizien. En Languedoc oriental,

4 Fig. 15 - Les ensembles composant le style 3 du Chasséen en Languedoc oriental. Variante $3 a$ : 30 - Nîmes - Mas de Vignole 4, F8056 (Mv3 : $n^{\circ} 1$ à 23) ; variante $3 b: 34$ - Villeneuve-lès-Maguelonne - grotte de La Madeleine (coll. Barral), quelques éléments des c. III à I (Md 1 à $5: n^{\circ} 24$ à 30) (Dessins et mise au net : S. Lancelot et C. Georjon, INRAP).

Sets for chassean style 3 of Eastern Languedoc, 3a variant: 30 - Nîmes - Mas de Vignole 4, F8056 (Mv3 : $n^{\circ} 1$ to 23$)$; $3 b$ variant: 34 - Villeneuvelès-Maguelonne - cave of La Madeleine (Barral collection), some elements of layers III to I (Md 1 to $5: n^{\circ} 24$ à 30) 


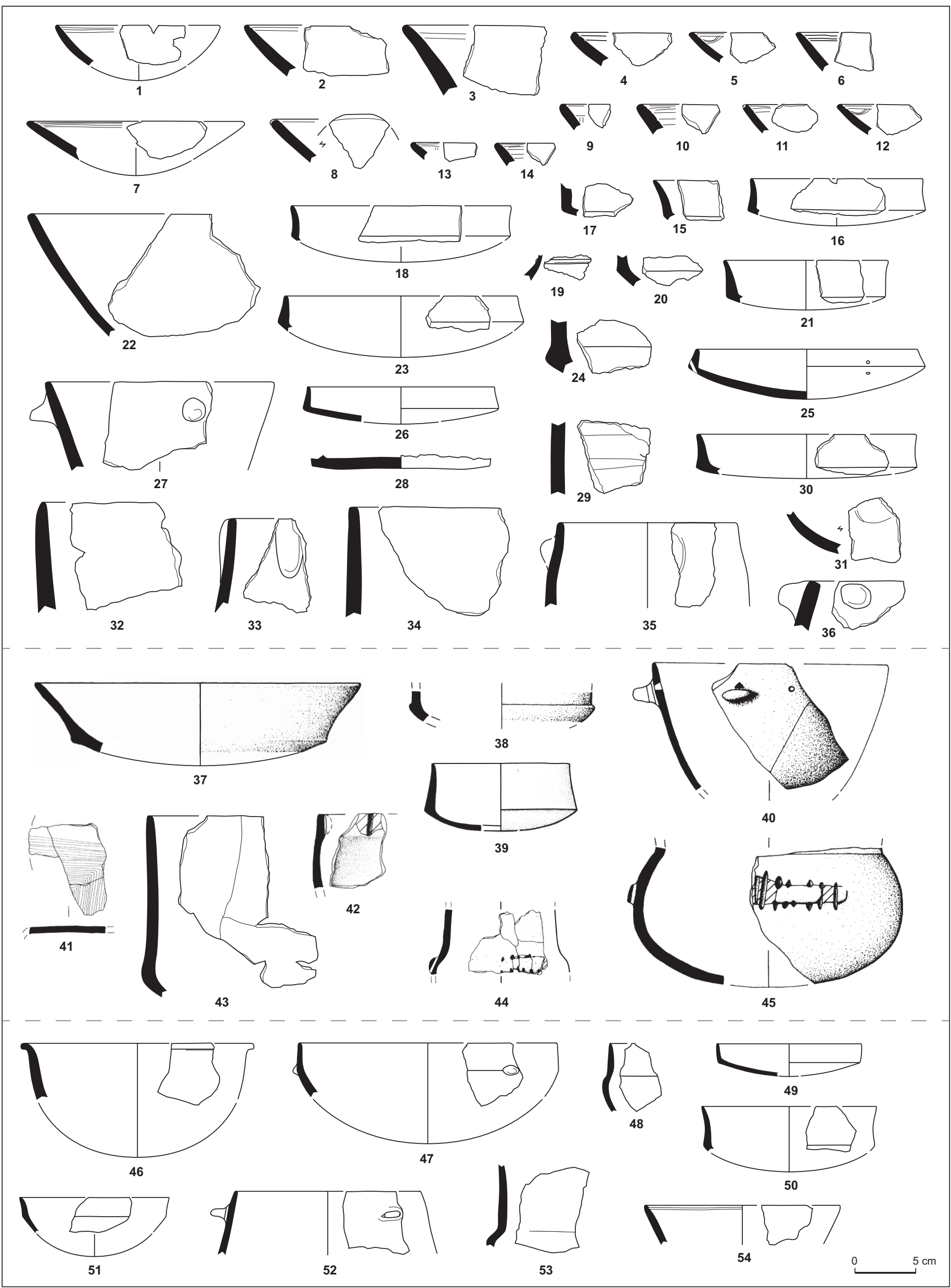




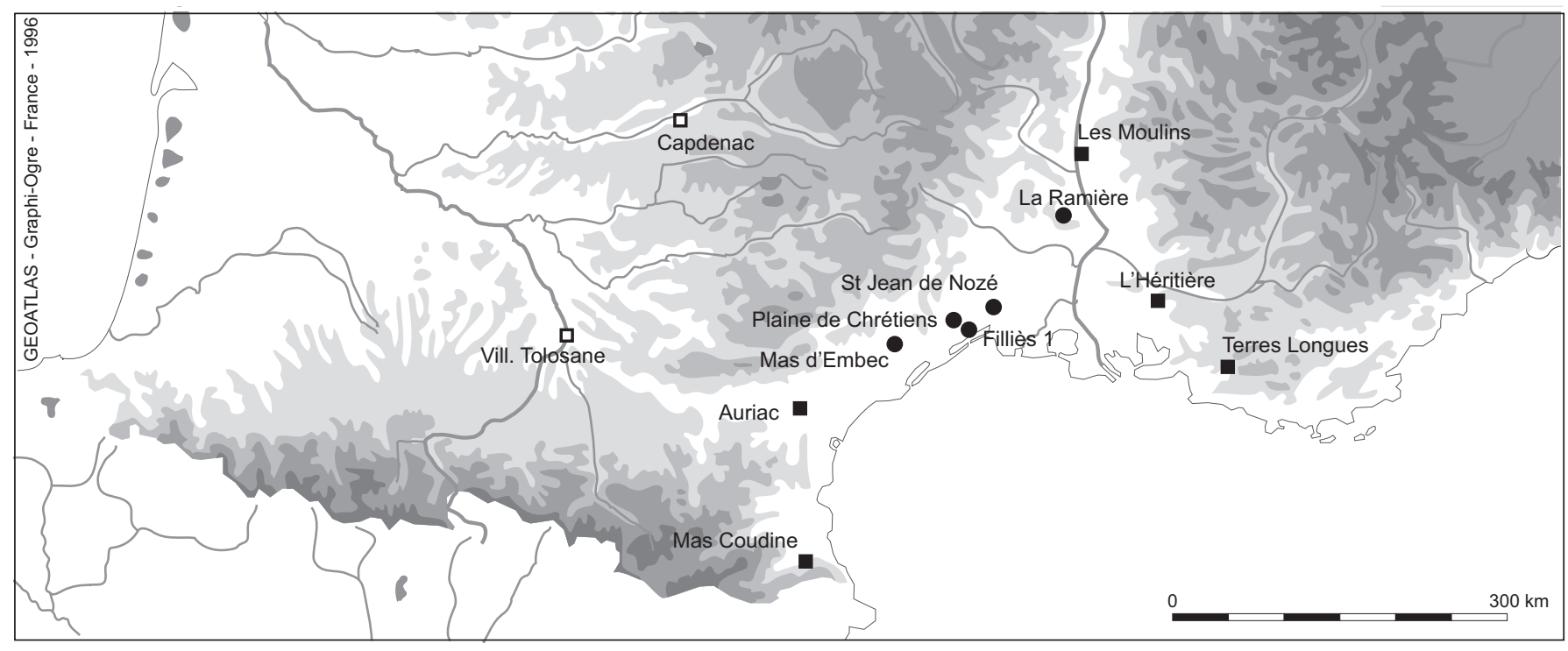

Fig. 18- Cartographie du style 4 du Chasséen en Languedoc oriental (ronds noirs) et comparaisons hors de la zone d'étude (carrés noirs). Autres gisements cités dans le texte (carrés blancs).

Sites for chassean style 4 of Eastern Languedoc (black circles) and comparisons outside of the study area (black squares). Other sites listed in the text.

Vaucluse et Drôme, ces gabarits sont remplacés par des gobelets à épaulement bas et col haut et par des tulipiformes. D'autre part, la présence dans la variante $5 \mathrm{~b}$, de lignes de boutons sur bord, de boutons uniques ou doubles sur rupture de pente et de cols à sillon (Raffègues, notamment), crée un rapprochement avec la Provence (phase D2 ; Lepère, 2012) et la Drôme (Chasséen D1 ; Beeching, 2002) ; notamment avec les séries de La Combe à Caromb dans le Vaucluse et Le Trou Arnaud à Saint-Nazaire-le-Désert dans la Drôme (Léa et al., 2004a ; Beeching, 2002).

Aucun indice ne permet de connaître le rapport chronologique entre les variantes $5 \mathrm{a}$ et $5 \mathrm{~b}$, en l'absence de stratigraphies. En Provence, la succession entre les phases D1 et D2 a été appréhendée grâce à la séquence de la grotte $\mathrm{C}$ à Baudinard (Lepère, 2012). Cependant, les assemblages de ce gisement ne sont pas les comparaisons les plus pertinentes pour le style $5 \mathrm{du}$ Languedoc oriental.

\section{LE NÉOLITHIQUE MOYEN NON CHASSÉEN}

\section{LE NÉOLITHIQUE MOYEN I}

Les ensembles représentant cette période en Languedoc oriental (fig. 22), tous inédits et situés à Nîmes, sont très res- treints (Mas de Vignole 4, F8325, F4029 et F3064, Bassin aval du Vistre de la Fontaine, couche archéologique et Mas des Abeilles II, F278, F308 et F499).

On retiendra la présence de grands récipients à paroi ouverte ou verticale, à bord épaissi ou ceint d'un large cordon peu saillant. Les anses en ruban sont, avec les languettes, les seuls éléments de préhension représentés. Les décors sont des cordons horizontaux lisses larges uniques ou doubles, des cordons étroits verticaux ou courbes et des impressions sur lèvre. Les pâtes des céramiques de ces ensembles incorporent très fréquemment des inclusions mates de forte taille, probablement en calcite. Ce mode de dégraissage pourrait correspondre à celui qui a été décrit pour la série des couches 2-7, post-cardiales ou pré-chasséennes, de La Font des Pigeons à Châteauneuf-lèsMartigues dans les Bouches-du-Rhône (Dangel, 1997).

En Languedoc occidental, cette phase est connue à travers les séries mal documentées de Font Juvénal à Conques dans l'Aude, ensemble 12, de la grotte Gazel à SallèlesCabardès, phase IV, de la grotte IV de Saint-Pierre-de-la-Fage, couches $2 \mathrm{a}-2 \mathrm{~b}$, et de Camprafaud à Ferrière-Poussarou dans l'Hérault, niv. 15-14 (Guilaine, 1997a ; Arnal, 1983 ; Rodriguez, 1983). On retrouve l'exclusivité des formes simples, les décors de cordons lisses, les bouteilles à col sinueux. Les impressions, notamment sur lèvre sont présentes à Camprafaud.

En Provence, les ensembles pré-chasséens plus fournis

Fig. 17 - Les ensembles composant le style 4 du Chasséen en Languedoc oriental. 34 - Montpellier, Plaine de Chrétien, F39 (Pc : $n^{\circ} 1$ à 36) ; 30 - Roquemaure, La Ramière, éléments les plus représentatifs des structure F5083 et F8048 (Rm1 et Rm2: $n^{\circ} 37$ à 45); 34 - Lunel, Saint Jean de Nozé, éléments représentatifs issus des prospections ( $n^{\circ} 46$ à 54) (Dessins et mise au net : S. Lancelot et C. Georjon, INRAP).

Sets for chassean style 4 of Eastern Languedoc. 34 - Montpellier, Plaine de Chrétien, F39 (Pc: $n^{\circ} 1$ to 36 ); 30 - Roquemaure, La Ramière, significant elements of structures F5083 and F8048 (Rm1 et Rm2: $n^{\circ} 37$ to 45); 34 - Lunel, Saint Jean de Nozé, significant elements found during the prospecting activity ( $n^{\circ} 46$ to 54 ) (Drawings: S. Lancelot and C. Georjon, INRAP). 


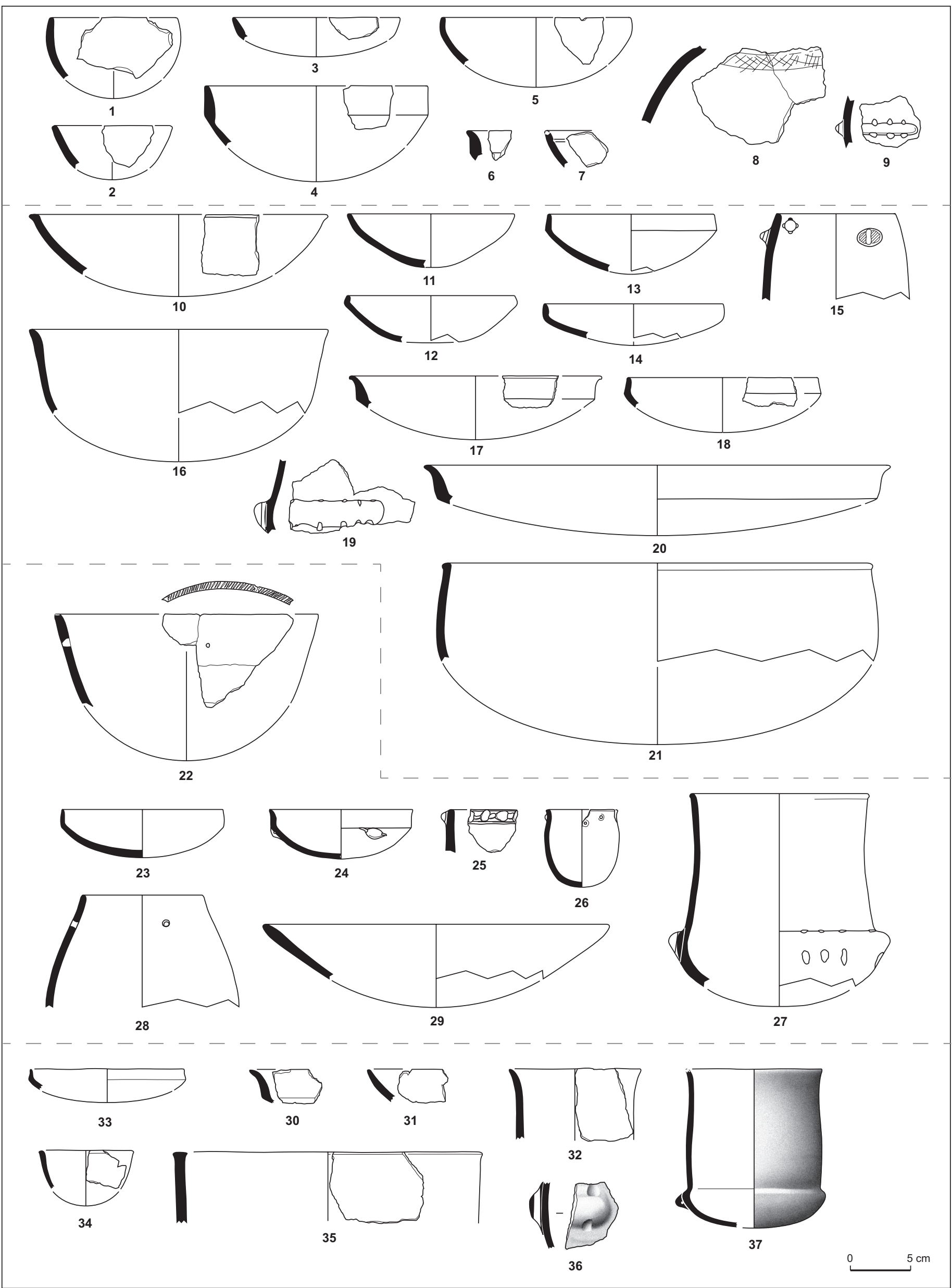


4 Fig. 19 - Les éléments composant le style 5a du Chasséen en Languedoc oriental. 34 - Castelnau-le-Lez - Jardins de Vert Parc, synthèse des formes de PT1020 ( $n^{\circ} 1$ à 9) ; 30 - Cavillargues - La Roquette, FS1439 ( $n^{\circ} 10$ à 21) et FS1361 ( $n^{\circ} 22$ à 29$)$; 30 - Nîmes - Mas de Vignole IV, FS4094 ( $n^{\circ} 30$ à 37) (Dessins et mise au net: C. Georjon et S. Lancelot, INRAP).

Sets for chassean style 5a of Eastern Languedoc. 34 - Castelnau-le-Lez - Jardins de Vert Parc, synthesis of F1020 ( $n^{\circ} 1$ to 9); 30 - Cavillargues La Roquette, FS1439 ( $n^{\circ} 10$ to 21) and FS1361 ( $n^{\circ} 22$ to 29); 30 - Nîmes - Mas de Vignole IV, FS4094 ( $n^{\circ} 30$ to 37) (Drawings: C. Georjon and S. Lancelot, INRAP).

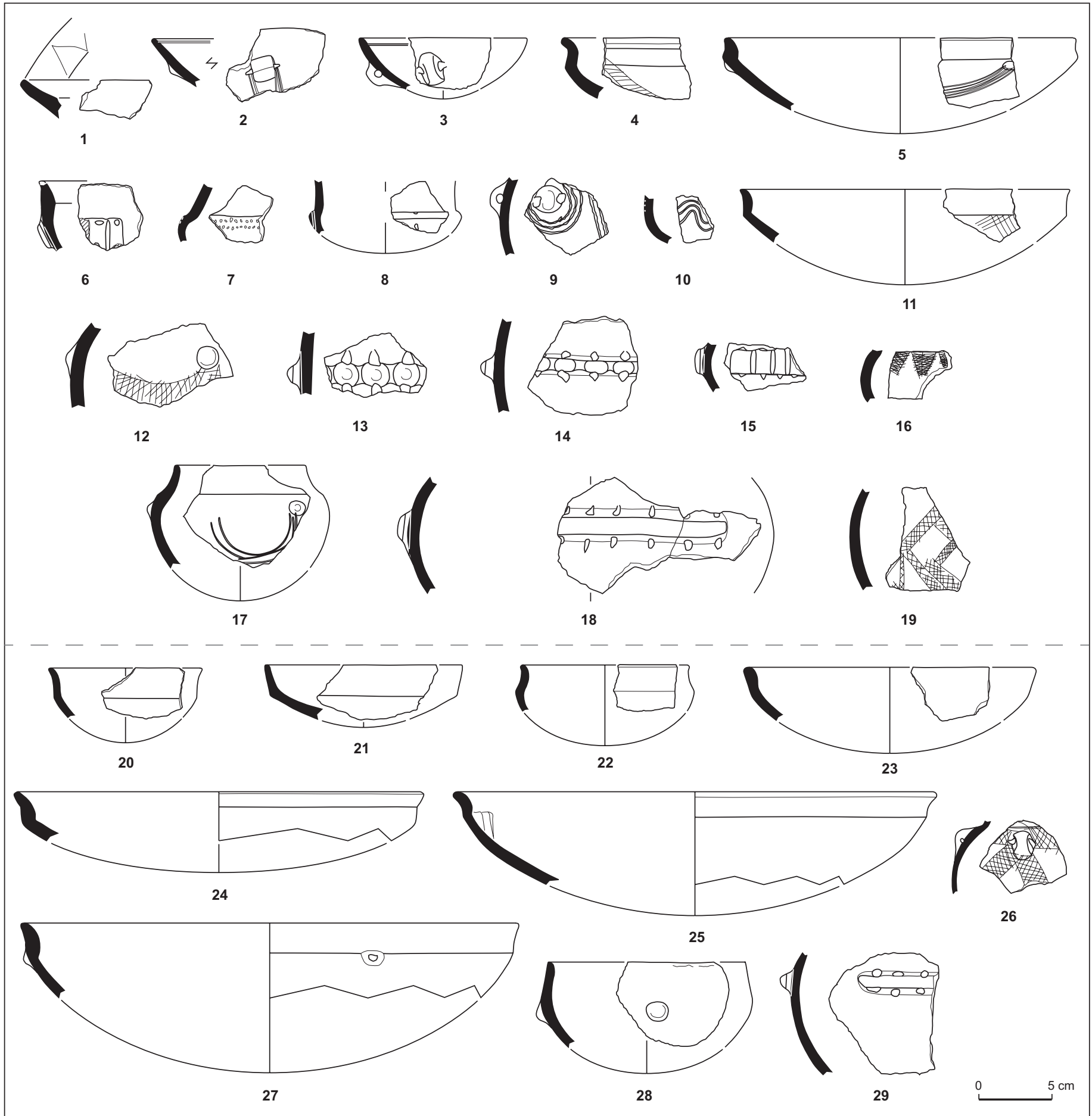

Fig. 20 - Éléments céramiques chasséens des grottes gardoises rattachables au style $5 b$ (prospections et ramassages sélectifs anciens de la collection du Musée d'Histoire Naturelle de Nîmes). 30 - Sainte-Anastasie, grotte de Saint Vérédème ( $n^{\circ} 1$ à 19$) ; 30$ - Tharaux, grotte des Fées ( $n^{\circ} 18$ à 29) (Dessins et mises au net : C. Georjon, INRAP).

Ceramic elements of the caves of Gardon valley attributed to the style $5 b$ (selective ancient collection of the Museum of natural history fo Nîmes). 30 - Sainte-Anastasie, cave of Saint Vérédème ( $n^{\circ} 1$ to 19); 30 - Tharaux, cave of Les Fées ( $n^{\circ} 18$ to 29) (Drawings : C. Georjon, INRAP). 


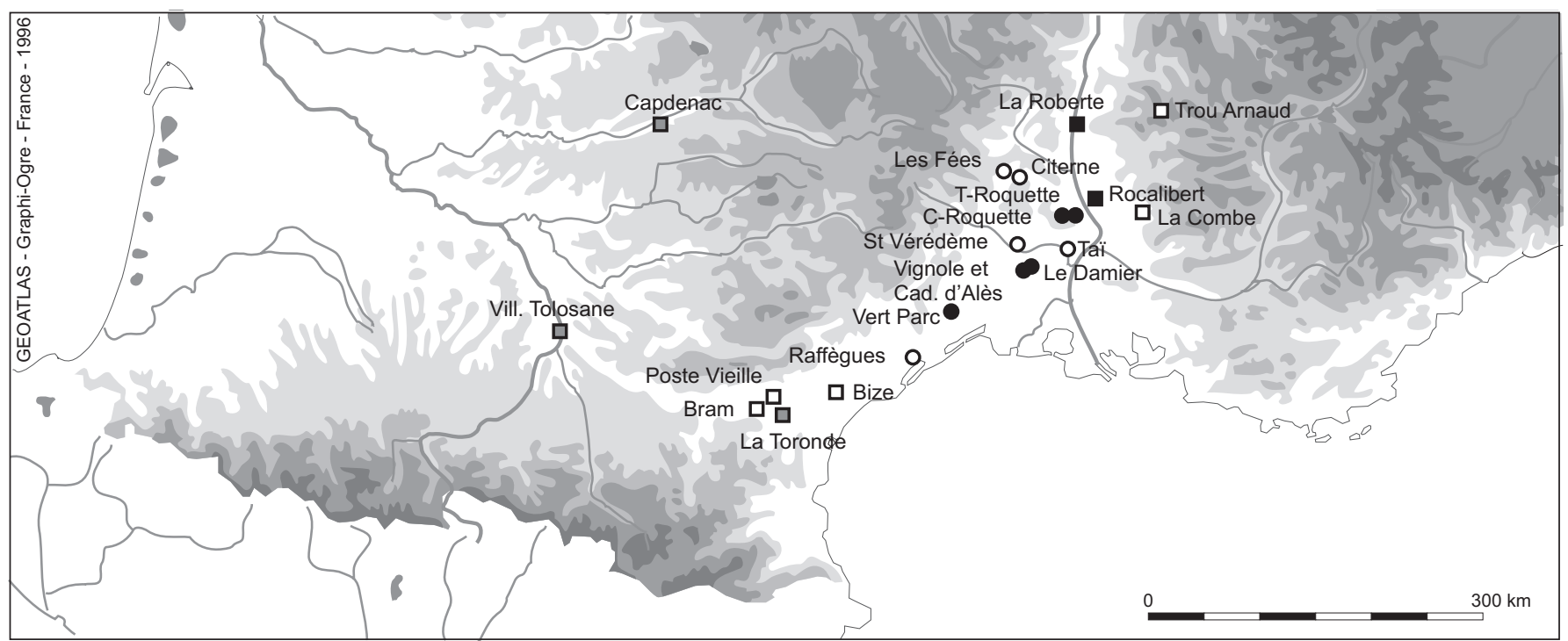

Fig. 21 - Cartographie du style 5 du Chasséen en Languedoc oriental (ronds) et sites de comparaison hors de la zone d'étude (carrés). En noir : variante 5 a et sites de comparaison; en blanc : variante $5 b$ et sites de comparaison; en gris : autres sites.

Sites for chassean style 5 of Eastern Languedoc (circles) and comparisons outside of the study area (squares). In black: $5 a$ variant and sites outside of the study area; in white: $5 b$ variant and sites outside of the study area; in grey: other sites.

montrent une certaine disparité typologique ; cela va de formes et décors dérivés du Cardial-Epicardial (La Font des Pigeons couches 2-7, grotte de l'Adaouste à Jouques dans les Bouchesdu-Rhône couches 2-6 et Fontbrégoua à Salernes dans le Var, couches 38-30 ; Dangel, 1997 ; Luzi et Courtin, 2001 ; Escalon de Fonton et Onoratini, 1991 ; Onoratini et al., 1997) à des formes carénées plus franchement chasséennes (Fontbrégoua, couches 38-30), avec des éléments ornementaux originaux dont certains sont d'influence italique (Villa Giribaldi à Nice, première phase, Binder et al., 2008 ; Lepère, 2009), en passant par un certain dépouillement comme à Escanin II aux Bauxde-Provence dans les Bouches-du-Rhône, couches 3 à 6 selon les secteurs (Lepère, 2002). Outre des différences de substrats culturels, ces variations sont peut-être d'ordre chronologique. Par exemple, la série d'Escanin, par sa sobriété décorative et l'absence de caractères proprement chasséens, pourrait faire office d'intermédiaire entre Pré- et Proto-Chasséen. En outre, à Fontbrégoua, série la mieux documentée, il n'est pas certains que l'ensemble des éléments attribués à cette période soit bien homogène (Luzi et Courtin, 2001) ; les formes carénées nous paraissent en effet appartenir au Proto-Chasséen ou au Chasséen ancien.

Nous retiendrons, pour nos comparaisons, la présence d'une grande marmite ouverte et d'impressions sur la lèvre à La Font des Pigeons; de cordons lisses à Escanin et à la grotte de l'Adaouste. Sur ce dernier site, de fins cordons verticaux évoquent bien ceux du Mas des Abeilles II.

\section{UN ENSEMBLE DE LA FIN DU NÉOLITHIQUE MOYEN}

Les données dont nous disposons pour appréhender la fin du Chasséen se résument à la petite série de La Grange des Merveilles I, couche archéologique, à Rochefort-du-Gard dans le Gard (Monnet et al., 2002). Les seuls points communs avec le Chasséen sont les pâtes encore très fines et la présence de carènes et d'un micro-ressaut. Il est vrai que les carènes ne disparaissent toutefois pas complètement au début du Néolithique final. Les comparaisons se font essentiellement avec des ensembles provençaux, notamment avec Les Juilléras à Mondragon et La Blaoute à Crillon-le-Brave dans le Vaucluse, le niv. Supérieur de Château Blanc à Ventabren et les niv. 14A à E du Mourre de la Barque à Jouques dans les Bouches-du-Rhône (Lemercier, 2002 ; Buisson-Catil, 1996 ; Hasler et al., 1998 ; Van Willigen, 2010). Ces ensembles ont en commun avec La Grange des Merveilles, outre l'absence de décors et la monotonie des éléments de préhensions, la présence de coupes et jattes à carène haute et de coupes simples à bord déversé. Au Mourre de la Barque comme aux Merveilles, les micro-ressauts sont connus et les boutons ronds sont les principaux types de préhension.

\section{LA CHRONOLOGIE ABSOLUE DU LANGUEDOC ORIENTAL}

En Languedoc oriental, nous disposons d'une vingtaine de dates pour caler les épisodes pré-chasséen et chasséen. Quelques unes ont été écartées :

- celles de la grotte de la Madeleine,couches X et VII qui présentent des écart-types de \pm 200 à 250 ans ;

- celle de Raffègues dont on ne sait à quelle couche ou structure elle se rapporte ;

- celle de la Baume Bourbon à Cabrières dans le Gard, couche 4 dont le mobilier, restreint, n'a pas été étudié dans le cadre de cette étude ;

- celle de la Baume d'Oullins au Garn dans le Gard, couche 5, pour les mêmes raisons ;

- celle de du Mas d'Embec, structure 1 qui n'est pas cohérente. Parmi les vingt dates retenues (fig. 24), quinze sont inédites. 


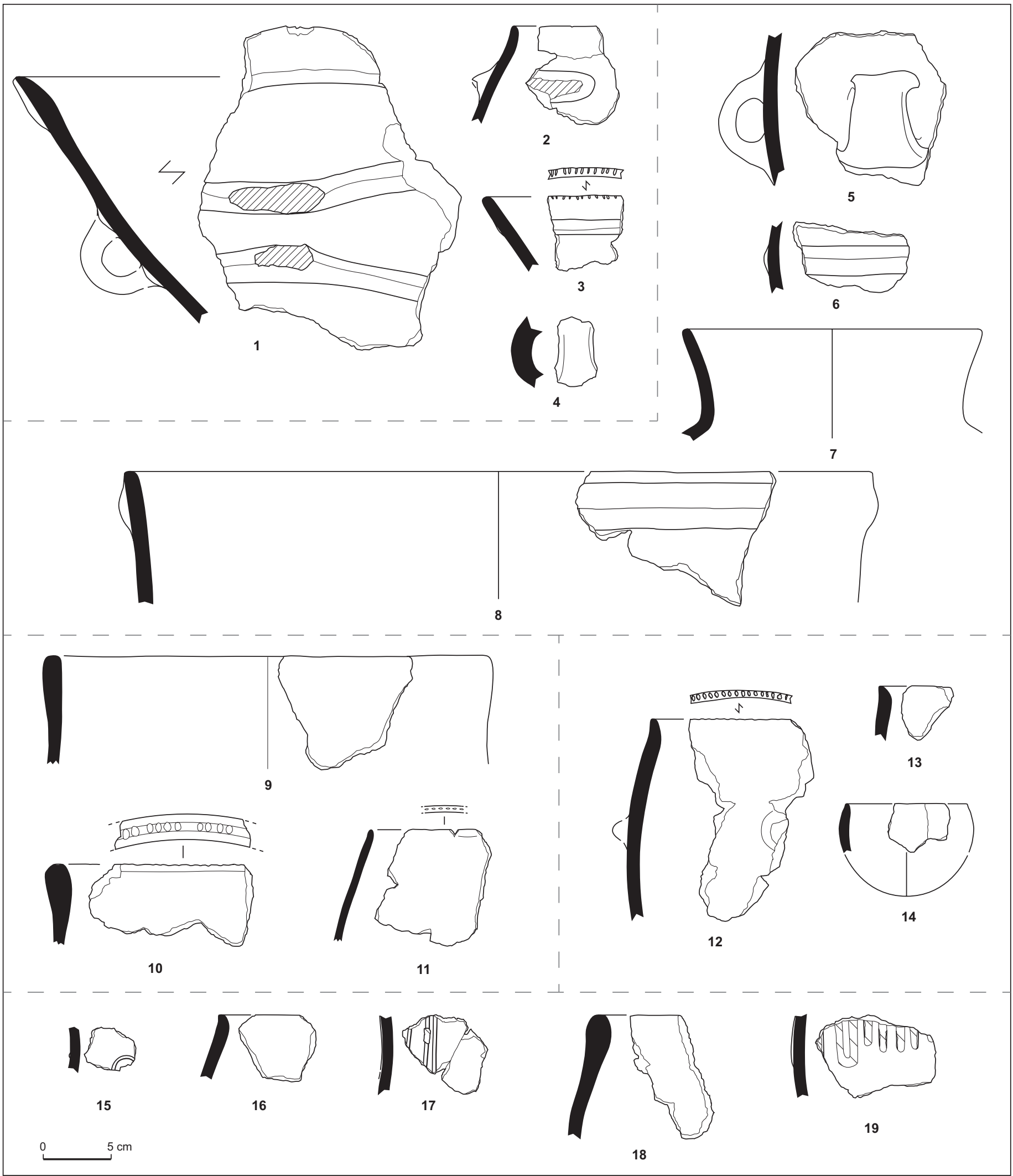

Fig. 22 - Éléments céramiques du Néolithique moyen 1 en Languedoc oriental. 30 - Nîmes, Mas de Vignole 4, F3064 ( $n^{\circ} 1$ à 4 ), F8325 ( $n^{\circ} 5$ à 8), F4029 ( $n^{\circ} 9$ à 11); 30 - Nîmes - Bassin aval du Vistre de la Fontaine, couche archéologique ( $n^{\circ} 12$ à 14); 30 - Nîmes, Mas des Abeilles II, niveau archéologique ( $n^{\circ} 15$ à 18), F1011 (n019) (Dessins et mise au net : C. Georjon, INRAP).

Ceramic elements of the Middle Neolithic I of Eastern Languedoc. 30 - Nîmes, Mas de Vignole 4, F3064 (n 1 to 4 ), F8325 ( $n^{\circ} 5$ to 8 ), F4029 ( $n^{\circ} 9$ to 11); 30 - Nîmes - Bassin aval du Vistre de la Fontaine, archaeological layer ( $n^{\circ} 12$ to 14); 30 - Nîmes, Mas des Abeilles II, archaeological layer ( $n^{\circ} 15$ to 18$)$, F1011 ( $\left.n^{\circ} 19\right)$ (Drawings : C. Georjon, INRAP). 
Tabl. II - Tableau des datations Radiocarbone calibrées retenues pour le Néolithique moyen en Languedoc oriental. Oxcal v4.1.7 Bronk Ramsey (2010) ; r.5 Atmospheric data from Reimer et al. (2009).

Table of Radiocarbon dating calibrated for the Middle Neolithic of Eastern Languedoc.

Treize d'entre elles, celles de La Clau et de Mas de Vignole 4, ont été très récemment effectuées sur os, en vérifiant la validité des contextes de prélèvement (fosses) et en choisissant soigneusement les échantillons à dater. Toutes les autres dates ont été réalisées sur des charbons ; les échantillons de Port Ariane, La Grange des Merveilles et de Mas d'Embec ont été prélevés dans des foyers, celui de Vert Parc provient d'un puits. Nous ne connaissons pas la nature exacte de ces prélèvements de charbons, quant aux contextes, ils paraissent fiables. Au Mas d'Embec et à Vert Parc, les échantillons sont associés à un mobilier significatif.

À Port Ariane, les corrélations stratigraphiques de F21013, F21074 et F21076 d'où proviennent les mesures avec les couches archéologiques ne posent pas de problème, même si seul F21013 renferme des tessons; ces structures sont très proches des niveaux fouillés et leur niveau d'ouverture correspond à la «nappe inférieure » ayant livré la majorité du mobilier. Le cas de F22005 est moins évident ; il est plus éloigné des secteurs décapés et sans connexion avec eux ; de plus il n'a livré aucun mobilier (Coye, 2007). L'ancienneté de F21074 ne peut s'expliquer.

À La Grange des Merveilles, le foyer daté est situé à $40 \mathrm{~m}$ au sud de la nappe de vestiges et, en dehors du fait qu'il se situe au même niveau que cette nappe, aucun indice ne permet de relier les deux faits archéologiques (Monnet et al., 2002).

Pour le site de La Clau, l'une des deux structures qui apparaissent dans les analyses statistiques n'a malheureusement pas pu être datée faute de matériaux. Le mobilier de certains autres faits datés a pu être rapproché des styles définis. Dans cette série de mesures, deux phases principales se dégagent, correspondant plus ou moins à ce qui a été vu dans les analyses typologiques.

Un grand déséquilibre apparaît entre les différentes périodes chasséennes datées (fig. 23) : 15 mesures concernent les deux styles du Chasséen ancien tandis que trois dates seulement s'attachent aux trois styles des phases récentes. Le Néolithique moyen non chasséen bénéficie de deux mesures seulement. Ce déséquilibre rend très fragile toute interprétation de la chronologie. Dans la suite de ce chapitre, les intervalles chronologiques calibrés seront donnés à un sigma.

L'étirement chronologique des phases anciennes, malgré de faibles écart-types, s'explique à la fois par le nombre important de mesures qui les concernent, mais aussi bien sûr par le plateau de la courbe de calibration dans lequel elles s'inscrivent. En l'état, les éléments du Néolithique moyen I et le Chasséen ancien s'enchaînent bien ; le premier étant situé entre 4555 et 4460 av. J.-C., le second recouvrant une plage de temps entre 4340 et 3985 av. J.-C. Les dates du style 1 s'étirent entre 4340 et 4075 av. J.-C., cependant, un lot d'ensembles de La Clau montre qu'il pourrait ne pas dépasser 4250 av. J.-C. Le style $2 \mathrm{a}$, qui ne bénéficie que d'une seule mesure, apparaît contemporain du style 1 (4310-4070 av. J.-C.). Le style 2b se situerait entre 4225 et 3985 av. J.-C., si l'on en croit la série des dates les plus récentes de La Clau. L'unique date disponible pour le style 3 concerne la variante $3 \mathrm{a}$. Elle se place nettement après 4000 av. J.-C. (3965-3805 av. J.-C.). Le style 4 se situerait entre 3787 et 3705 av. J.-C., en contemporanéité relative avec le style 5a (3820-3650 av. J.-C.), les deux ne bénéficiant que d'une seule mesure chacun. Enfin le Néolithique moyen de la Grange des Merveille est bien postérieur aux manifestations chasséennes (3640 et 3520 av. J.-C.).

\section{PREMIÈRES COMPARAISONS ENTRE LES SÉRIATIONS LITHIQUE ET CÉRAMIQUE}

Une précédente étude (Léa, 2004a) ainsi que de nombreux travaux, encore inédits pour la plupart, effectués dans le cadre du PCR de Nîmes coordonné par Jean-Yves Breuil ou dans le cadre de rapports de fouilles préventives, ont mené à la proposition d'un phasage à partir de la caractérisation des assemblages lithiques du Languedoc oriental. Une comparaison des données entre les phasages respectifs des mobiliers céramique et lithique peut donc d'ores et déjà être réalisée (fig. 24). Néanmoins, cette comparaison se heurte à un obstacle de taille. Les sites qui ont livré des assemblages fournis, à la fois d'un point de vue céramique et lithique, sont rares. Aussi, les sites clefs permettant de définir clairement des étapes dans l'évolution chronoculturelle du Chasséen du Languedoc oriental sont assez peu souvent les mêmes du point de vue et de la céramique et du lithique. De plus, les assemblages lithiques sont représentés en effectifs très inégaux selon les sites et certains d'entre eux sont parfois peu abondants (tabl. II). Il s'agit donc ici seulement de rendre compte des changements les plus nets, en attendant qu'une confrontation plus fine soit possible notamment grâce à la réalisation de plus nombreuses dates radiocarbones.

Afin d'effectuer cette comparaison entre évolution des styles céramique et lithique, il convient de reprendre succinctement les styles lithiques qui sont ici utilisés (Léa, 2004a). Le style A caractérise les assemblages lithiques constitués exclusivement par des productions d'éclats non standardisés réalisées aux dépends de matières premières locales ou sub-locales. Les pièces esquillées et les grattoirs sont, parmi les supports transformés, les outils les plus fréquents. Le style B caractérise les assemblages au sein desquels le recours aux matières premières locales reste important, en parallèle de l'introduction des sites aux réseaux de diffusion des silex bédouliens non chauffés du Vaucluse, sous différentes formes (éclats, lames débitées par percussion indirecte ou pression et produits finis comme certaines armatures, notamment de grands trapèzes sur lames larges ou éclats). Dans certains assemblages (comme celui de Saint-Antoine), du silex oligocène rubané d'Apt-Forcalquier est de même importé et utilisé pour la confection de lamelles par pression. Le style $\mathrm{C}$ se traduit par l'apparition des productions lamellaires, débitées par pression en silex bédouliens chauffés $\mathrm{du}$ Vaucluse, qui deviennent prépondérantes dès le style $\mathrm{C} 1$. Le recours aux matières premières locales est anecdotique. Des burins sur lamelles et de rares chanfreins sont présents. 


\begin{tabular}{|c|c|c|c|c|c|c|c|c|}
\hline Dpt & Commune & Site & Contexte & Matériau & Code labo. & Date BP & cal BP $68 \%$ & cal BP 95\% \\
\hline \multirow{4}{*}{ 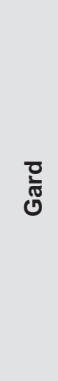 } & Nîmes & $\begin{array}{l}\text { Grange des } \\
\text { Merveilles }\end{array}$ & $\mathrm{F} 2$ & charbon & ARC-1393 & $4777 \pm 50$ & $\begin{array}{l}3609-3522(57 \%) \\
3639-3620(11,2 \%)\end{array}$ & $\begin{array}{l}3654-3498(82 \%) \\
3436-3377(13,4 \%)\end{array}$ \\
\hline & Nîmes & $\begin{array}{l}\text { Mas de Vign. } 4 \text { et } \\
\text { Cad. d'Alès (MV4) }\end{array}$ & F8325 & os & POZ-35552 & $5689 \pm 41$ & $4554-4459$ & $\begin{array}{l}4682-4635(5 \%) \\
4620-4449(90,2 \%)\end{array}$ \\
\hline & Nîmes & $\begin{array}{l}\text { Mas de Vign. } 4 \text { et } \\
\text { Cad. d'Alès (CD) }\end{array}$ & F3162 & os & ERL-15112 & $5350 \pm 36$ & $\begin{array}{l}4312-4301(4 \%) \\
4260-4224(17,3 \%) \\
4206-4162(22,2 \%) \\
4130-4071(24,7 \%)\end{array}$ & $\begin{array}{l}4323-4290(9,9 \%) \\
4266-4143(52,6 \%) \\
4138-4052(32,9 \%)\end{array}$ \\
\hline & Nîmes & $\begin{array}{l}\text { Mas de Vign. } 4 \text { et } \\
\text { Cad. d'Alès (MV4) }\end{array}$ & F8056 & os & POZ-35551 & $5102 \pm 51$ & $\begin{array}{l}3966-3914(26,7 \%) \\
3878-3804(41,5 \%)\end{array}$ & $\begin{array}{l}4033-4026(0,6 \%) \\
3991-3776(94,8 \%)\end{array}$ \\
\hline \multirow{16}{*}{$\begin{array}{l}\frac{\omega}{3} \\
\frac{\pi}{\frac{\pi}{\pi}} \\
\frac{0}{I}\end{array}$} & $\begin{array}{l}\text { Castelnau- } \\
\text { le-Lez }\end{array}$ & Jardins de Vert Parc & F1020 & charbon & ARC-1973 & $4980 \pm 50$ & $\begin{array}{l}3905-3880(8,3 \%) \\
3800-3695(59,9 \%)\end{array}$ & $\begin{array}{l}3942-3856(20,6 \%) \\
3842-3838(0,4 \%) \\
3820-3653(74,3 \%)\end{array}$ \\
\hline & Gigean & La Clau & F1124 & os & POZ-38094 & $5440 \pm 40$ & $\begin{array}{l}4340-4313(26,7 \%) \\
4301-4260(41,5 \%)\end{array}$ & $\begin{array}{l}4358-4232(95 \%) \\
4187-4182(0,4 \%)\end{array}$ \\
\hline & Gigean & La Clau & F1122 & os & POZ-38092 & $5440 \pm 40$ & $\begin{array}{l}4340-4313(26,7 \%) \\
4301-4260(41,5 \%)\end{array}$ & $\begin{array}{l}4358-4232(95 \%) \\
4187-4182(0,4 \%)\end{array}$ \\
\hline & Gigean & La Clau & F1106 & os & POZ-38090 & $5440 \pm 40$ & $\begin{array}{l}4340-4313(26,7 \%) \\
4301-4260(41,5 \%)\end{array}$ & $\begin{array}{l}4358-4232(95 \%) \\
4187-4182(0,4 \%)\end{array}$ \\
\hline & Gigean & La Clau & F1044 & os & POZ-38088 & $5420 \pm 40$ & $4332-4258$ & $\begin{array}{l}4352-4228(90,2 \%) \\
4200-4170(4,5 \%) \\
4090-4080(0,8 \%)\end{array}$ \\
\hline & Gigean & La Clau & F1060 & os & POZ-38089 & $5370 \pm 40$ & $\begin{array}{l}4325-4286(19,7 \%) \\
4268-4228(24,4 \%) \\
4201-4168(15,6 \%) \\
4127-4120(2,6 \%) \\
4094-4078(5,9 \%)\end{array}$ & $\begin{array}{l}4331-4221(51,8 \%) \\
4211-4150(21,8 \%) \\
4134-4054(21,8 \%)\end{array}$ \\
\hline & Gigean & La Clau & F1120 & os & POZ-38091 & $5360 \pm 40$ & $\begin{array}{l}4321-4292(12,2 \%) \\
4265-4226(20,9 \%) \\
4204-4166(19 \%) \\
4129-4114(5,8 \%) \\
4100-4074(10,3 \%)\end{array}$ & $\begin{array}{l}4328-4280(16,8 \%) \\
4274-4146(51,4 \%) \\
4136-4052(27,3 \%)\end{array}$ \\
\hline & Gigean & La Clau & F1054 & os & POZ-38132 & $5310 \pm 40$ & $\begin{array}{l}4230-4194(16,2 \%) \\
4176-4146(13,5 \%) \\
4136-4054(38,5 \%)\end{array}$ & $\begin{array}{l}4258-4039(93,4 \%) \\
4016-4000(2 \%)\end{array}$ \\
\hline & Gigean & La Clau & F1195 & os & POZ-38134 & $5260 \pm 40$ & $\begin{array}{l}4226-4205(9,9 \%) \\
4164-4130(17 \%) \\
4112-4102(4,1 \%) \\
4072-4034(19,5 \%) \\
4025-3991(17,7 \%)\end{array}$ & $\begin{array}{l}4231-4193(14,3 \%) \\
4176-3978(81,1 \%)\end{array}$ \\
\hline & Gigean & La Clau & F1105 & os & POZ-38133 & $5260 \pm 40$ & $\begin{array}{l}4226-4205(9,9 \%) \\
4164-4130(17 \%) \\
4112-4102(4,1 \%) \\
4072-4034(19,5 \%) \\
4025-3991(17,7 \%)\end{array}$ & $\begin{array}{l}4231-4193(14,3 \%) \\
4176-3978(81,1 \%)\end{array}$ \\
\hline & Gigean & La Clau & F1004 & os & POZ-38161 & $5250 \pm 40$ & $\begin{array}{l}4224-4207(7,6 \%) \\
4159-4132(12,8 \%) \\
4068-3984(47,8 \%)\end{array}$ & $\begin{array}{l}4230-4196(11,7 \%) \\
4174-3972(83,7 \%)\end{array}$ \\
\hline & Lattes & Port Ariane & 21074 & charbon & LY-1023 (OXA) & $5510 \pm 65$ & $\begin{array}{l}4448-4415(17,3 \%) \\
4405-4327(46,4 \%) \\
4282-4272(4,5 \%)\end{array}$ & $4489-4238$ \\
\hline & Lattes & Port Ariane & 21013 & charbon & LY-1022 (OXA) & $5400 \pm 65$ & $\begin{array}{l}4340-4228(54,3 \%) \\
4200-4170(10 \%) \\
4126-4122(1,1 \%) \\
4090-4080(2,8 \%)\end{array}$ & $4351-4051(95,4 \%)$ \\
\hline & Lattes & Port Ariane & 21076 & charbon & LY-9699 & $5375 \pm 35$ & $\begin{array}{l}4326-4285(25,1 \%) \\
4270-4228(28,8 \%) \\
4198-4172(13 \%) \\
4088-4084(1,3 \%)\end{array}$ & $\begin{array}{l}4332-4223(61,2 \%) \\
4207-4156(18,7 \%) \\
4131-4066(15,4 \%)\end{array}$ \\
\hline & Lattes & Port Ariane & 22005 & charbon & LY-1024 (OXA) & $5315 \pm 60$ & $\begin{array}{l}4232-4144(34,2 \%) \\
4137-4052(34 \%)\end{array}$ & $\begin{array}{l}4324-4288(5,8 \%) \\
4268-4034(84,1 \%) \\
4026-3991(5,5 \%)\end{array}$ \\
\hline & $\begin{array}{l}\text { Saint- } \\
\text { Pargoire }\end{array}$ & Mas d'Embec & st 7 & charbon & LY-9239 & $4980 \pm 50$ & $3783-3704$ & $\begin{array}{l}3908-3878(5,3 \%) \\
3802-3656(90,1 \%)\end{array}$ \\
\hline
\end{tabular}




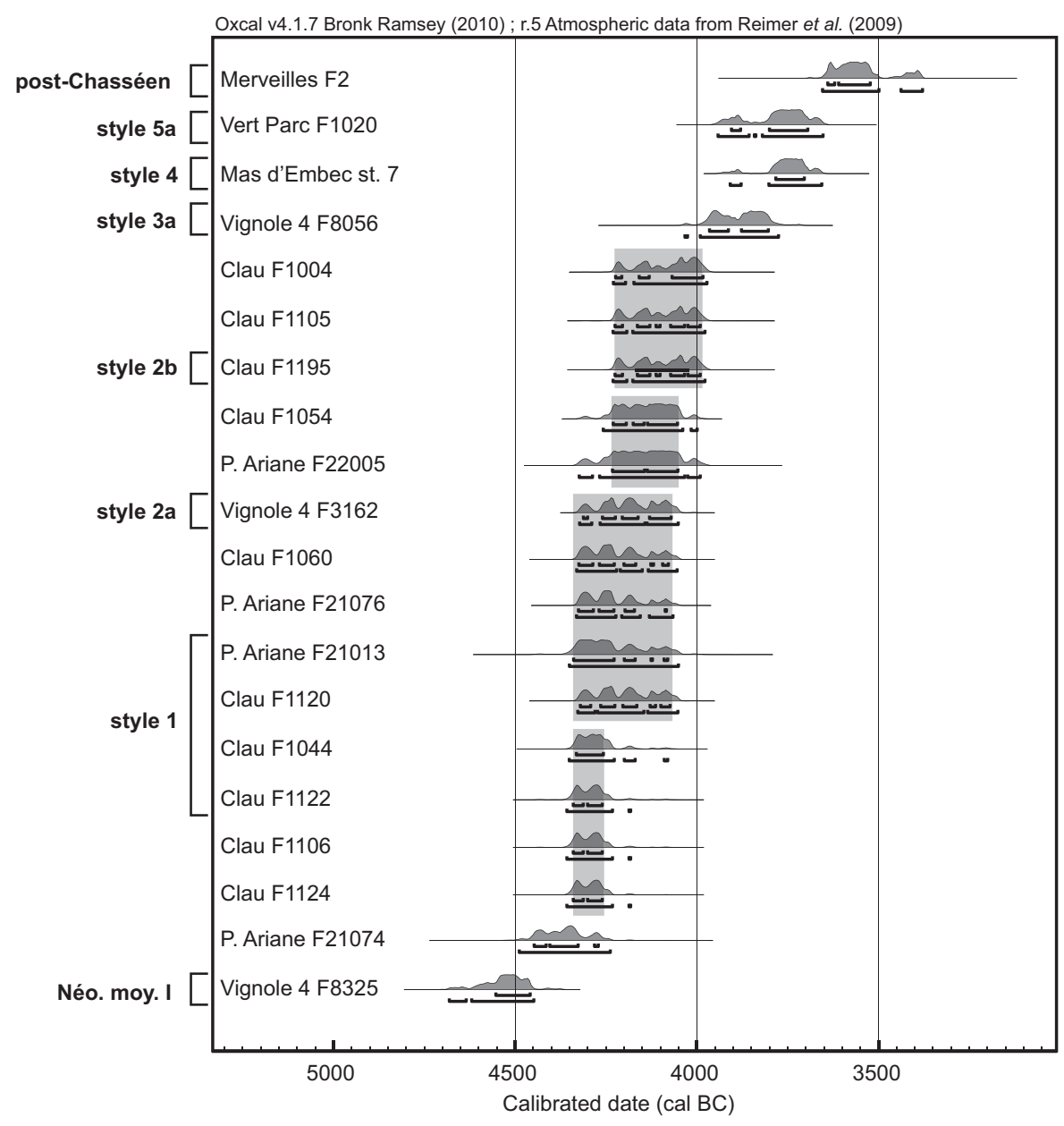

Fig. 23 - Compilation des datations du Néolithique moyen en Languedoc oriental calibrées avec la courbe Intercal09.

Compilation of Radiocarbon dating for the Middle Neolithic of Eastern Languedoc calibrated with Intercal09 curve.

Les grands trapèzes sur lames larges en silex bédouliens non chauffés sont absents alors que de petits géométriques sur éclats ou lamelles en silex bédoulien chauffé sont bien représentés.

Globalement, cette première comparaison de l'évolution des styles céramiques avec celle des styles lithiques montre que les phases définies à partir de la céramique et du lithique se correspondent en partie (fig. 26). Il est notamment important de remarquer que les deux mobiliers attestent de concert deux très nets changements lors du passage du style céramique $3 \mathrm{a}$ au style céramique $3 \mathrm{~b}$ correspondant au passage du style $\mathrm{B}$ au style $\mathrm{C}$ lithique, c'est-à-dire à l'introduction des sites aux réseaux de diffusion des silex bédouliens chauffés du Vaucluse. Ce changement, qui se situe autour de 4000 av. J.-C., est noté bien au-delà $\mathrm{du}$ Languedoc oriental, comme dans l'aire liguro-provençale par exemple, et a d'ailleurs été à l'origine de la proposition de l'emploi du terme Chasséen récent pour tout ce qui se passait à partir du début du IV e millénaire (Binder et al., 2008). De même, le passage du style céramique $3 \mathrm{~b}$ au style 4 , correspond au passage entre le style $\mathrm{C} 1$ et le style $\mathrm{C} 2$ lithique (cf. infra).

Cependant, d'importants décalages existent dans l'évolution des styles céramiques et lithiques. Un premier décalage est perceptible en ce qui concerne les phases anciennes. L'industrie lithique atteste une évolution plus lente, et certaines étapes, bien marquées par le mobilier céramique (passage du Néolithique moyen I au style céramique 1, du style 1 au style 2 ou encore du style 2 au style 3a), ne sont pas traduites par le mobilier lithique. En effet, alors que les caractères proprement chasséens se développent tôt dans la céramique, permettant la distinction entre Néolithique moyen I ante-Chasséen et style 1 chasséen en correspondance avec la chronologie absolue (cf. infra), les assemblages lithiques (style A) ne montrent encore aucun caractère chasséen. Tous les assemblages lithiques qui se rapportent au style 1 céramique des sites du Mas des Abeilles II, du Mas de Vignole, de Port Ariane, de La Clau III, des Jardins de Vert Parc, de Saint-Antoine secteur I, sont caractérisées par un débitage d'éclats non standardisés, à la percussion directe dure ou sur enclume, réalisés aux dépends de matières premières locales, notamment issues des anciennes terrasses rhodanoduranciennes des Costières de Nîmes. Notons qu'il a d'ailleurs déjà été démontré que ces productions sur galets locaux de quartz ou de silex, dans le cadre de débitage d'éclats, n'avaient pas de valeur chrono-culturelle, puisqu'on peut les retrouver jusqu'aux phases récentes du Chasséen de manière anecdotique (Léa, 2004b). Dans ce contexte, les pièces esquillées et les grattoirs sont prédominants. Ce style lithique A pourrait donc avoir duré longtemps, il prédomine au moins depuis 4500 jusqu'à 4200-4100 av. J.-C. (Léa et al., 2004b). Ces remarques font échos à celles déjà exprimées dans une précédente synthèse (Léa 2004a, p. 174) et rappelle les réflexions formulées concernant une certaine inertie notée en Languedoc occidental lors du processus de néolithisation (Vaquer 1989, p. 194). La situation paraît en revanche différente en Provence où le silex bédoulien non chauffé joue un rôle important dans les assemblages lithique dès le Pré-chasséen notamment dans le cadre d'une production d'éclats selon un mode bifacial (Binder 1990, 1998).

Les premiers changements dans l'industrie lithique (style B 


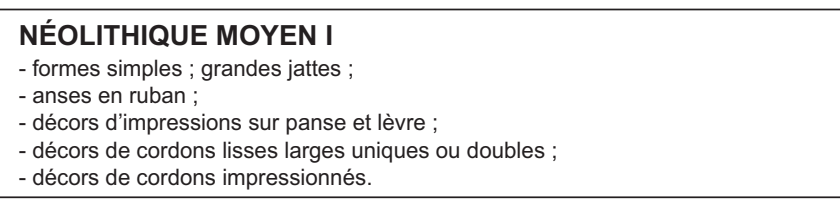

\section{CHASSÉEN STYLE 1}

- assiettes à marli et à socle ; formes simples ;

- anses en ruban

- décors de cordons impressionnés ;

- décors de cordons lisses larges uniques ;

- décors de cannelures jointives ;

- décor rayé/quadrillé sur assiettes à marli et à socle.

\begin{tabular}{ll}
\hline CHASSÉEN STYLE 2a & CHASSÉEN STYLE 2b \\
- assiettes à marli et à socle ; & - assiettes à marli et à socle ; \\
- marmites à inflexion basse ; & - assiettes et coupes à bourrelet ou \\
- bols et marmites à carène basse ; & méplat pré-oral interne ; \\
franche et paroi haute ; & - marmites à inflexion basse ou carène \\
- marmites à paroi sinueuse ; & adoucie et paroi haute ; \\
- bouteilles à col sinueux; & - marmites et gobelets à paroi sinueuse ; \\
- anses en ruban ; & - bols et marmites globuleux ; \\
- cordons horizontaux à perforation & - jarres à bandeau pré-oral externe ; \\
funiculaire (en mono-anses sur & - bouteilles à col sinueux; \\
carénés) ; & - anses en ruban ; \\
- cordons horizontaux biforés ou & - cordons horizontaux à perforation \\
multiforés contre la lèvre ou sur la & funiculaire ; \\
panse ; & - cordons horizontaux biforés ou \\
- décor rayé/quadrillé sur assiettes à & multiforés contre la lèvre ou sur la panse ; \\
marli et à socle. & - décor rayé/quadrillé sur assiettes à marli \\
& et à socle.
\end{tabular}

\section{CHASSÉEN STYLE $3 a$}

- assiettes à marli et à socle

- assiettes et coupes simples à bourrelet ou méplat pré-oral interne ;

- assiettes et coupes simples à sillon interne unique ;

- coupes carénées ; coupes, bols ou marmites à ressaut :

- jarres à bandeau pré-oral ;

- bouteilles à col sineux ou anguleux ;

- anses en ruban ; boutons prismatiques ; plaquettes multiforées ;

- décor rayé/quadrillé sur assiettes à marli et à socle ; moustaches incisées sur coupes.

\section{CHASSÉEN STYLE $3 b$}

- assiettes et coupes simples à sillon interne unique

- assiettes et coupes carénées ; coupes, bols ou marmites à ressaut ou

épaulement

- bouteilles à col sineux ou anguleux ;

- boutons prismatiques; plaquettes multiforées :

- moustaches incisées sur coupes ; décor rayé quadrillé sur bouteilles.

\section{CHASSÉEN STYLE 4}

- assiettes carénées :

- coupes à carène médiane ou basse et col demi-haut

- coupes, bols ou marmites à ressaut ou épaulement ;

- assiettes et coupes à sillons internes uniques ou multiples accompagnant ou

non un décor rayé quadrillé périphérique interne :

- bouteilles à col anguleux

- boutons prismatiques ;

- décor type Lagozza sur couvercles ;

- assiettes et coupes à moustaches incisées sur le fond ;

- décor rayé quadrillé sur la panse de bouteilles.

\section{CHASSÉEN STYLE 5}

- coupes carénées ou infléchies à col très court épaissi intérieurement ou non (cran) et carène haute ;

- assiettes carénées à col déversé ;

- gobelets et jarres à épaulement bas et col très haut ;

- bouteilles à col anguleux;

- bandeaux et cordons très saillants multiforés :

- sillons unique ou doubles :

- décors de moustaches, décors ondoyants, décors de guirlandes résillées sur le fond de coupes carénées

- décors rayés quadrillés sur bouteilles.

\section{STYLE A}

Recours à des matières premières locales, souvent aux dépens des anciennes terrasses rhodano-duranciennes des Costières de Nîmes ;

- Schémas de débitage divers et peu organisés : unidirectionel, multidirectionnel, sans organisation apparente, débitage sur enclume :

- Débitage d'éclats hétérométriques ;

- Prédominance des pièces esquillées et des grattoirs.

\section{STYLE B}

Recours à des matières premières locales ou sub-locales encore important - Introduction aux réseaux de diffusion des silex bédouliens non chaufés importation de lames débitées par percussion indirecte ou pression et d'éclats ayant pu servir de nucléus à éclats en silex bédouliens non chauffés; importation d'armatures trapézoïdales sur lame ou sur éclat larges type Berriac en silex bédoulien ;

- Dans certains cas (Saint-Aunès) importation de silex oligocène sous forme de lamelles.

\section{STYLE C1}

- Recours aux matières premières locales quasi nul ou exceptionnel dans le cadre d'un débitage d'éclats ;

- Introduction aux réseaux de diffusion de silex bédoulien chauffé : nucléus à plan de pression non incliné et lisse (Madeleine, couches I à III). Pas de nucléus de type quadrangulaire plat ;

- Importation de lames en silex bédouliens non chauffés.

\section{STYLE C2}

- Recours aux matières premières locales quasi nul ou exceptionnel dans le cadre d'un débitage d'éclats ;

- Introduction aux réseaux de diffusion de silex bédoulien chauffé : nucléus de type quadrangulaire-plat et de type «mixte»;

- Importation de lames en silex bédoulien non chauffé dans des proportions moindres que précédemment;

- Burins sur lamelles en silex chauffé, rares chanfreins sur lamelles ; apparition de petits géométriques sur lamelles ou éclats en silex bédoulien chauffé ; disparition des grands trapèzes sur lames large en silex bédoulien non chauffé ; disparition des grands trapèzes sur lames large en silex bédoulien non chauffé.

Fig. 24 - Confrontation entre les styles céramiques et les systèmes lithiques du Chasséen en Languedoc oriental.

Confrontation between ceramic and lithic styles for Chassey culture of Eastern Languedoc. 


\begin{tabular}{|c|c|c|c|c|}
\hline Dpt & Commune & Site & Bibliographie & $\begin{array}{l}\text { Effectif } \\
\text { lithique }\end{array}$ \\
\hline \multirow{3}{*}{ गु } & Roquemaure & La Ramière & Léa 2004a & 149 \\
\hline & Jonquières-Saint-Vincent & Jarnègues & Léa 2004a & 331 \\
\hline & Montfrin & Le Réal & Léa 2004a & 155 \\
\hline \multirow{9}{*}{ 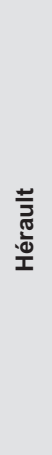 } & Lattes & Lattes Port-Antique & Léa 2004a & 4957 \\
\hline & Lattes & Port-Ariane & Léa 2004a & 1390 \\
\hline & Béziers & Le Crès & Léa 2004a & 919 \\
\hline & Mèze & Raffègues & Léa 2004a & 618 \\
\hline & Villeneuve-les-Maguelonne & La Madeleine & Léa 2004a & 216 \\
\hline & Saint-Aunès & Saint-Antoine & Furestier et al. sous presse & 2281 \\
\hline & Gigean & La Clau & Inédit & 493 \\
\hline & Castelnau-le-Lez & Vert-Parc & Briois et Léa 2003 & 299 \\
\hline & Montpellier & Jacques-Cœur & Jallot et al. 2000 & 13 \\
\hline$\frac{0}{\frac{0}{2}}$ & Carcassonnne & Champ du Poste & Inédit & 735 \\
\hline
\end{tabular}

Tabl. III - Les effectifs des ensembles lithiques de référence du Chasséen en Languedoc oriental et d'une série de comparaison dans l'Aude.

Staff of main chassean lithic sets of Eastern Languedoc and of one comparison set of Aude river basin.

lithique) ne se font sentir que pendant le style céramique 2 , avec l'introduction des sites aux réseaux de diffusion des silex bédouliens non chauffés. Malheureusement, cet évènement ne peut pas encore être daté de manière précise en Languedoc oriental ; nous ne savons pas dans quelle mesure il pourrait corrspondre à une évolution du style céramique 2 qui n'a pu être démontré (cf. infra, p. 61). L'importation de silex bédoulien non chauffé du Vaucluse se fait sous différentes formes : gros éclats ayant pu servir d'outils ou de nucléus à éclats, lames robustes débitées par pression ou percussion indirecte, et grandes armatures trapézoïdales façonnées sur lame large ou sur éclat comme sur les sites de Jacques-Cœur, La Madeleine, couches IX-IV, le Champ du Poste (F247, US247009) ou Saint-Antoine (certaines structures en zone 2). Ces armatures rappellent les exemplaires de Berriac Les Plots, de la tombe en caisson 2 de Dela Laïga à Cournanel, du coffre 4 de Najac, ou encore ceux de l'Arca de Calahons (Abelanet, 1970 ; Guilaine, 1959 ; 1962 ; Léa in Jallot et al., 2000 p. 287 et 290). Leurs retouches répondent généralement à une chronologie et une répartition très proche de celles issues de la technique dite « de Jean-Cros » (Tixier, 1979 ; Léa et al., 2009). Le recours aux matières premières locales reste important dans le cadre de productions d'éclats, que ce soit pour le silex ou le quartz. Enfin, il est intéressant de souligner la particularité du site de Saint-Antoine qui atteste, dans plusieurs structures, un débitage de lamelles par pression à partir d'un silex oligocène provençal, faisant référence à ce qui est connu plus à l'est, en Provence, à la grotte de l'Église supérieure dans le Var (Binder 1998). À l'ouest du Rhône, ce type de production reste pour l'heure très rare.

L'apparition du style C lithique caractérisé par les productions lamellaires en silex bédoulien chauffé, prend une place prépondérante au sein de tous les assemblages à partir de 4000 av. J.C., même si les lames en silex bédoulien non chauffés apparues durant le style B perdurent jusqu'à la fin du Chasséen dans les styles lithiques plus récents $\mathrm{C} 1$ et $\mathrm{C} 2$. À partir du style céramique 3 b et jusqu'au style céramique 5 inclus, tous les sites du Languedoc oriental sont intégrés aux réseaux de diffusion des productions en silex bédouliens chauffés vauclusiens qui deviennent largement majoritaires au sein des assemblages (phase lithique C). Comme partout dans le Midi de la France, on assiste à l'importation, depuis les ateliers du Vaucluse (Léa, 2004c), de préformes en silex bédouliens chauffés, vouées à un débitage de lamelles par pression, réalisé au sein de chacun des sites. L'importation de lames sous forme de support en silex bédouliens non chauffés ne disparait cependant pas, même si leur représentativité au sein des assemblages devient moins importante. Les galets locaux ne sont plus exploités que ponctuellement, toujours pour un débitage d'éclats. C'est pendant cette phase qu'apparaissent les petites géométriques, les burins et les chanfreins sur lamelles en silex bédouliens chauffés (Léa, 2004a) à l'instar de ce qui est connu en Provence (Binder, 1991 ; Léa, 2003). Les grands trapèzes sur lames larges ou éclats ont en revanche disparu.

La stratigraphie de la grotte de La Madeleine illustre formidablement ce passage du style lithique B au style lithique C (Léa 2004a). Dans cette stratigraphie, les couches I à IX ont livré du mobilier lithique. Dans les couches IX à IV (appelé Ensemble II inférieur), les éléments en silex bédouliens non chauffés sont bien représentés $(n=50)$ alors que trois petits fragments de lamelles en silex bédouliens chauffés semblent provenir des niveaux supérieurs de manière post-dépositionelle. Notons ici, qu'à la différence des résultats céramiques, les couches IV et V ne se distinguent pas au sein de cet Ensemble II (elles n'ont livré aucun élément chauffé). Dans l'Ensemble I supérieur, c'est-à-dire dans les couches I à III, les lamelles en silex bédouliens chauffés sont très bien représentées $(n=47)$ contrairement à ce qui était attesté dans l'Ensemble II inférieur, même si les lames en silex bédouliens non chauffés ne disparaissent pas. À la Madeleine, le style de débitage de ces lamelles en silex bédouliens chauffés est particulier et mène à la distinction d'un style $\mathrm{C} 1$, correspondant au style céramique 3 b. Le style $\mathrm{C} 1$ se rapproche du faciès semi-conique décrit dans les assemblages de Provence (Binder, 1991 ; Léa, 2003) - le plan de pression n'est pas incliné, les bords et les nervures sont convergents mais contrairement à ce dernier, le plan de pression des nucléus de La Madeleine n'est presque jamais facetté, il est quasi exclu- 
sivement lisse (Léa, 2004a). Or, il est important de noter ici qu'en Languedoc oriental, le style de débitage semi-conique à plan de pression facetté « provençal » ne paraît pas bien représenté. Peut-être cela est-il dû à un problème d'échantillonnage des séries ou bien à un style régional particulier ? La question mérite d'être posée dans la mesure où ce style de débitage semiconique à plan de pression facetté a été retrouvé plus à l'ouest dans le toulousain à Villeneuve-Tolosane dans l'assemblage du puits (Léa et al., 2007) ainsi que sur le site de Saint Michel du Touch à Toulouse (Galin, 2012).

Le style lithique C2, associé aux styles céramiques 4 et 5 , correspond au faciès quadrangulaire-plat défini à partir d'assemblages de Provence (Binder, 1991 ; Léa, 2003). Ce style est bien représenté en Languedoc oriental sur de nombreux sites tels Lattes Port-Antique, Mas d'Embec et Raffègues. Néanmoins, s'il est relativement fréquent, il n'est presque jamais exclusif car il est souvent associé à un style de débitage de type « mixte » caractérisé par des nucléus semi-coniques à plan de pression lisse incliné (Léa 2004a). Ce style de débitage peut avoir différentes significations, comme l'introduction de préformes d'un type particulier (cf. infra), ou bien il peut attester un changement en cours de débitage : un nucléus quadrangulaire-plat peut, comme le montrent des remontages sur le site de Lattes (Léa 2004a), à la suite de réfections et corrections, devenir semi-conique en fin d'exploitation. Nous pensons par ailleurs que même en Provence où le style de débitage semi-conique est bien attesté, le passage d'un débitage semi-conique à plan de pression facetté à un débitage quadrangulaire plat se fait très rapidement. Sur le site de La Ramière qui appartient au style céramique 4, seul le débitage de type mixte est représenté. Ce constat peut s'expliquer par deux hypothèses non exclusives : 1/ il s'agit d'une fin d'exploitation des nucléus quadrangulaires-plats comme nous l'avons évoqué ; ou 2/ le style quadrangulaire-plat n'est maîtrisé que par certains ateliers vauclusiens car sa mise en forme demande un savoir-faire particulier. Notons que cette deuxième hypothèse trouve de sérieux arguments en contexte d'atelier dans la zone de production vauclusienne (Léa, inédit). Ainsi, il nous semble encore prématuré de scinder cette grande phase lithique $\mathrm{C} 2$ en plusieurs étapes, et le manque de stratigraphies régionales fait de même ici cruellement défaut. Enfin, la fin du Chasséen dans cette région, comme dans d'autres, est encore mal caractérisée du point de vue lithique. Les sites candidats pour cette période sont quasi inexistants à l'heure actuelle. Seul le site de la Grange des Merveilles pourrait être invoqué. Or, sur ce site, la présence de lames en silex bédoulien gris non chauffé importées sous forme de supports (Monnet et al., 2002) contraste totalement avec le système chasséen de la phase $\mathrm{C}$. L'effondrement du système complexe de diffusion des productions spécialisées en silex bédouliens chauffés du Vaucluse pourrait donc être relativement brutal.

Quatre styles lithiques se dégagent donc. Le style A correspond du point de vu céramique au Pré-Chasséen et au style chasséen 1 et à une partie du style chasséen 2 identifiés par la typologie céramique : la diffusion du silex bédoulien n'est pas encore effective ; les industries lithiques reposent exclusivement sur un débitage d'éclats hétérométriques à partir de diverses matières premières locales. Le style $\mathrm{B}$, correspondant à une partie du style céramique 2 ainsi qu'au style $3 a$, voit l'amorce de la diffusion du silex bédoulien non chauffé, comme dans les ensembles lithiques associés au style des Plots. Avec les deux styles $C$ (styles céramiques $3 b, 4$ et 5 ) apparaissent les productions lamellaires en silex bédoulien chauffé qui se divisent en style $\mathrm{C} 1$ (correspondant au style céramique $3 b$ ) et style C2 (correspondant au style céramique 4 et 5).

Pour l'heure il parait difficile d'aller plus loin dans notre réflexion. Il faut dire que les stratigraphies avec datations fines à la clef font ici cruellement défaut. Les fouilles anciennes tout comme les fouilles récentes, au sein desquelles les choix de datations ne sont pas souvent réalisés en fonction de problématiques chrono-culturelles ou lithiques, ne permettent pas de disposer d'un corpus de dates suffisant pour caler précisément tel ou tel évènement lithique.

\section{CORRÉLATION DES PÉRIODISATIONS DU CHASSÉEN MÉRIDIONAL}

Pour proposer un tableau de comparaison des chronologies méridionales (fig. 25), nous avons opéré une sélection des datations radiocarbone, en fonction de leur cohérence avec les séries céramiques auxquelles elles font référence, mais nous avons également tenté de replacer les ensembles non datés qui nous ont servi de comparaison. Les relations entre les différents domaines géographiques méridionaux sur la base des comparaisons stylistiques ont été reportées.

\section{NÉOLITHIQUE MOYEN I ET PROTO-CHASSÉEN}

En Languedoc, dans la Drôme, en Provence et en Ligurie, les manifestations attribuées au Pré-Chasséen prennent fin aux environs de 4400 av. J.-C.

$\mathrm{Au}$ Vistre de La Fontaine, au Mas de Vignole 4 (F8325, F4029 et F3064), au Mas des Abeilles II, à la grotte IV de SaintPierre-de-la-Fage couches 2a-2b, à Camprafaud couches 15-14, à la grotte Gazel phase IV, à La Font des Pigeons couches 7-2 et à la grotte de l'Adaouste couches 6-2, certaines marmites ouvertes sont encore massives, le reste du corpus est composé de bols et marmites plus réduits à profil sinueux et parfois amorce de col; les préhensions sont essentiellement composées d'anses en ruban et de languettes. Les décors de cordons divers sont bien déployés sur les vases, accompagnés parfois de rubans d'impressions ou de boutons, en héritage du Néolithique ancien. Ces éléments sont clairement étrangers au Chasséen et appartiennent au Néolithique moyen I. Les ensembles concernés sont tous trop restreints pour permettre d'estimer leur relation chronologique et pour se prêter à une définition culturelle. Le Néolithique moyen I ante-Chasséen demeure très difficile à appréhender compte tenu de la rareté des sites et de la pauvreté des mobiliers qui lui sont rattachés. Sur ce point, le contraste est saisissant avec le domaine septentrional qui donne l'impression d'un bouillonnement culturel après la fin du Rubané, avec pléthore de sites.

Le Proto-Chasséen qui s'installe ensuite se caractérise par une meilleure représentation des vases plus petits et des vases bas et ouverts (coupes, bols) entrainant l'affinement 


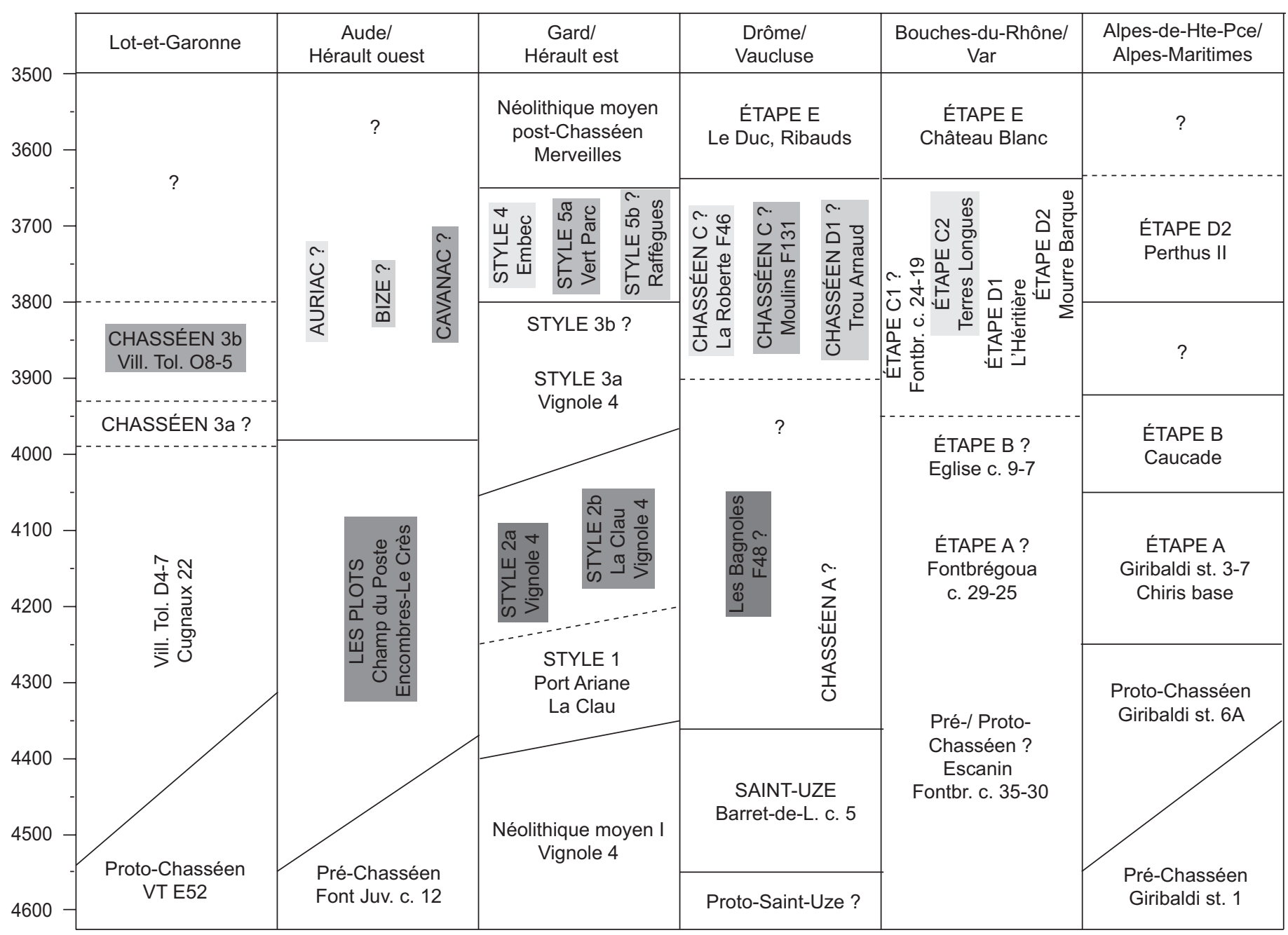

Fig. 25 - Proposition de synchronisation des chronologies régionales du Midi. Les affinités stylistiques inter-régionales les plus marquées sont indiquées par des nuances de gris (d'après Beeching, 1995 ; 2002 ; Beeching et al., 1997 ; Binder et al., 2008 ; François, 2002 ; 2007 ; Gandelin, 2011 ; Monnet et al., 2002 ; Vaquer, 1991, 1998 ; Lepère, 2009 ; 2012).

Proposal of synchronizing the Southern chronologies. Great affinities are indicated by colors.

des parois, la diversification nette des types de préhension, la multiplication des suspensions à perforation sous-cutanée. Dès lors, comme l'écrit très justement C. Lepère, « les particularités morphologiques et volumétriques des vases reflètent des "thèmes fonctionnels" que l'on peut isoler » (Lepère, 2002, p. 64). La céramique, qui ne reçoit plus que rarement des décors en relief, prend son caractère « lisse ».

En Quercy, Languedoc et Provence rhodanienne, les formes demeurent le plus souvent sans segmentation et les premiers vrais éléments chasséens apparaissent de façon irrégulière selon les sites (cordons multiforés, coupes à marli et à socle ornées, jarres à bandeau pré-oral). On trouve encore de rares cordons lisses ou impressionnés. En Languedoc oriental au moins, la céramique conserve de la période précédente le dégraissant mat grossier. Si les industries lithiques n'intègrent, en Languedoc, aucun des caractères chasséens, la situation semble différente en Provence (Luzi et Courtin, 2001 ; Lepère, 2002 ; Binder et al., 2008). Les séries qui représentent le mieux cette période sont Port Ariane, La Clau, Saint Antoine secteur 1, Escanin 2 et Fontbrégoua couches 38-30 .

Contrairement à ce que nous observons en Languedoc oriental, les récipients carénés - mono-ansés pour la plupart sont déjà présents à cette période au moins en Ligurie (Giribaldi), en Provence (Fontbrégoua couches 38-30). L'origine de ces formes dans le Néolithique inférieur italique (Fiorano Fagnigola) est établie. Si l'on s'extrait du domaine strictement méridional, la stratigraphie du Gardon dans l'Ain montre la présence de ces carénés mono-ansés dans le Saint-Uze récent (4450-4200 av. J.-C.). En Auvergne, la série proto-chasséenne de Pontcharaud 2 en fournit de nombreux exemplaires, mais ils sont soit sans préhension, soit à mono-barrette miniaturisée. Notons enfin que dans cette étape, les carénés bas et larges sont très rares (un exemplaire à Fontbrégoua et peut-être d'autres en Ligurie ?). La chronologie absolue est difficile à appréhender pour cette phase. Les dates sont rares et peu cohérentes. On

5. Le rassemblement de ces couches ne garantit pas l'homogénéité de la série qui peut présenter des éléments du Néolithique moyen I. 
peut estimer qu'elle se termine aux environs de 4250 av. J.-C.

\section{LE CHASSÉEN ANCIEN}

Il faut tout d'abord reconnaître que les données disponibles sur le Chasséen ancien du Midi sont très diverses d'une région à l'autre. Aux nombreux sites de l'Aude et du Languedoc oriental, s'opposent la rareté et l'indigence des ensembles provençaux, drômois et quercynois, tandis que Villeneuve-Tolosane s'impose comme l'unique référence de cette période pour le bassin de la Garonne.

Comme l'a déjà noté C. Lepère (2012), on retrouve un bon nombre des caractéristiques du Chasséen ancien dans l'ensemble du Midi, ce qui révèle probablement « une tradition commune » (ibid., p. 534) bien ancrée. Cette phase semble bien longue (4300-4000 av. J.-C. au minimum), mais elle s'inscrit dans le plateau de la courbe de calibration, ce qui peut expliquer en partie une telle durée. On peut toutefois se demander si elle ne pourrait pas être séquencée. Cette question, pour laquelle les datations radiocarbones ne sont d'aucun secours pour l'instant, paraît également difficile à trancher au moyen de la typologie céramique. En effet, si le Languedoc oriental montre la possibilité d'une variabilité ( $2 \mathrm{a}$ et $2 \mathrm{~b}$ ), celle-ci n'est pas observée en Provence ni dans le Toulousain, où les caractéristiques principales des deux sous-styles que nous avons déterminés tendent à se mêler dans des faciès mixtes. Cette mixité ne semble pas absente dans notre zone d'étude comme le montre la fosse F9 de Jacques-Cœur, classée dans le style 2b (Les Plots) dans l'AFC. Ces éléments laissent supposer que les deux variantes distinguée pour le Languedoc oriental sont le fruit d'une variabilité « naturelle » voire fonctionnelle. Cependant, de nombreux ensembles du bassin de l'Aude et de l'Hérault occidentale, dont certains sont bien fournis, paraissent représenter l'équivalent d'une variante $2 \mathrm{~b}$ 《 pure » (style des Plots) et confortent les observations faites en Languedoc oriental. Si donc l'on veut creuser l'hypothèse d'un séquençage du Chasséen ancien, le point de départ serait de considérer les récipients carénés monoansés qui caractérisent la variante 2 a comme un héritage du Pré- ou Proto-Chasséen notamment provençal. Le style $2 b-$ ou style des Plots, dont les principaux marqueurs ont une postérité dans les premières phases du Chasséen récent (bourrelets ou méplats pré-oraux internes sur les coupes, jarres à bandeau pré-oral externe), s'installerait ensuite, soit en intégrant, soit en abandonnant les récipients carénés de 2a. Dans certains cas (Villeneuve-Tolosane, Capdenac-Le-Haut), les formes à carène basse et paroi développée semblent même connaître une longue postérité, contrairement à ce qui se passe en Languedoc oriental et en Provence.

Quoi qu'il en soit de cette hypothèse, la cohérence stylistique du Chasséen ancien des régions méridionales ne nous paraît pas remise en cause. Certes, l'influence du Montbolo est évidente à l'Ouest et celle des groupes italiques ne l'est pas moins à l'Est, mais ces variations géographiques demeurent limitées à quelques éléments, même s'ils sont parfois spectaculaires comme les décors peints des Bagnoles (Sargiano et al., 2010).

Aux marges septentrionales du domaine méridional, en Auvergne, les séries chasséennes du Pirou à Polignac (HauteLoire ; Houdré et Vital, 1979) et des Chambons à Prompsat (Puy-de-Dôme ; Vernet et Barthélémy, 1983 ; Jallet et Georjon, sous presse), se démarquent du Chasséen ancien méridional : les coupes à marli ne sont pas ornées, les coupes à bandeau ou bourrelet interne, de même que les jarres à bandeau sont absentes, les prises tunnelliformes épaisses des marmites et jarres n'ont pas d'équivalents dans le Midi, les carénés sont diversifiés et intègrent des formes basses et larges, très rares dans le Chasséen ancien du Midi. Si l'on ne peut juger des caractéristiques des industries lithiques du Pirou qui sont perdues, celles des Chambons montrent l'intégration à des réseaux qui n'ont rien à voir avec ceux du Midi.

De même, les niveaux 9 à 7 du camp de Chassey (Thévenot, 2005) ne peuvent, à notre sens, être associées sans difficulté au Chasséen ancien du Midi, comme le soutient une récente publication (Van Willigen et al., 2012). Les points de comparaison de cette série sont plutôt à chercher du côté de la Provence, semble-t-il, mais pas forcément dans les phases anciennes : les carénés bas et larges ne s'y développent pas avant la toute fin $\mathrm{du} 5^{\mathrm{e}}$ millénaire et les bols à micro-ressaut, surtout présents en Provence sont plus tardifs (Lepère, 2009 ; 2012). Les anses en boudin et les tunelliformes ou bobines épaisses sont rares dans le Midi. En outre, Chassey ne comporte aucune multitubulure et les cordons très longs multiforés saillants très répandus sur le site bourguignon sont, dans le Midi des éléments plutôt récents. Chassey appartient au Chasséen, distinct de celui du Midi, et la compréhension de ce style n'est actuellement pas facilitée par la rareté des sites chasséens qui l'environnent.

\section{LES PHASES RÉCENTES DU CHASSÉEN}

Dans les phases récentes, les processus d'évolution et de régionalisation semblent s'accélérer nettement. Dans un premier temps, on observe une continuité avec le style des Plots. De nombreux éléments perdurent dans le Quercy, dans le Garonnais, dans le style 3a du Languedoc oriental, en Provence et jusqu'en Ligurie (Caucade). Anses en ruban, coupes à méplat ou bourrelet pré-oral interne, jarres à bandeau pré-oral externe, assiettes à marli et à socle ornées, multitubulures persistent diversement dans les régions méridionales. Les récipients à carène basse et paroi ouverte deviennent fréquents et s'accompagnent de types encore rares (à carène haute et fond très bombé, larges à paroi courte) ; les coupes à sillon interne font une véritable apparition de même que les ressauts et les épaulements. Les éléments de suspension connaissent une nouvelle diversification (cordons saillants et bandeaux multiforés). Les industries lithiques sont marquées par l'apparition de la chauffe. Cette période pourrait se caler entre 4000 et 3900 av. J.-C. d'après les datations disponibles. Les principaux sites sont : Capdenac (niv. B), VilleneuveTolosane (Chasséen récent a), Mas de Vignole 4 (F8056) et Cadereau d'Alès (F1043 et F1055), La Madeleine, couches VI-IV, L'Église couches 9-7 et Caucade. Ce Chasséen est classique parce qu'il regroupe le plus grand nombre des caractères marquants de cette culture, comme le remarque M. Gandelin (2011). Il n'est pas homogène pour autant car l'exploitation des formes nouvelles donne lieu à des différenciations régionales : coupes à socle diverses et multitubures symbolisées par des traits verticaux dans le Garonnais et le Quercy, marmites larges à épaulement en Languedoc oriental, par exemple.

Les styles qui succèdent à ce Chasséen classique sont 
plus difficiles à organiser chronologiquement et géographiquement. À l'exception des toutes dernières manifestations du Chasséen et/ou du Post-Chasséen (phase E de Provence et Grange des Merveilles en Languedoc oriental), tous les styles récents semblent s'inscrire dans un même créneau chronologique entre 3900 et 3650 av. J.-C. env.

La variante $3 b$ du Languedoc oriental, essentiellement perçue à La Madeleine couches III-I, ne peut être mise en parallèle qu'avec la Provence (phase C1) et encore avec précaution. Les traits archaïques ont presque disparu. Les types de segmentation se multiplient (micro-ressauts, ressauts, épaulements anguleux ou adoucis) donnant de nouvelles formes. Les décors linéaires en creux se reportent sur les coupes simples ou segmentées (moustaches) et sur les bouteilles. Les différentiations géographiques commencent à prendre du poids; Le Garonnais et le Quercy paraissent se singulariser par rapport au Languedoc et à la Provence. La césure entre les variantes $3 \mathrm{a}$ et $3 \mathrm{~b}$ trouve un écho dans l'évolution du style lithique C.

Le style d'Auriac, correspondant à notre style 4 et à la phase C2 de Provence, s'étend largement, des Pyrénées orientales à la Provence et à la Drôme, mais demeure inconnu en Garonnais et en Quercy, malgré quelques affinités. Les assiettes carénées, généralement de petit volume, connaissent une embellie ; les formes à ressaut et épaulement incluent des gobelets à haut col, les décors internes des coupes se diversifient, les couvercles ornés apparaissent. Les principaux sites rattachés à ce style sont : Mas Coudine, Auriac, Plaine de Chrétiens, Les Moulins, L'Héritière et Terres Longues. Ce style paraît demeurer en contact avec la plaine littorale et la vallée du Rhône.

Probablement à la même période, à Villeneuve Tolosane (Chasséen récent b) et Capdenac dominent les récipients carénés à paroi haute ouverte et boutons prismatiques, même si les carénés bas sont présents. Une autre forme de prédilection est la bouteille à haut col et panse réduite, munie de tubulures sous-cutanées verticales ou de boutons bi- ou triforés verticalement. Les décors internes sur les coupes se diversifient de la même manière que dans l'Auriac et l'on trouve également des couvercles ornés. Les éléments « archaïques » persistent même s'ils sont raréfiés : coupes à bourrelet interne, jarres à bandeau pré-oral. Ce style et celui de Cavanac défini à partir du site éponyme de La Toronde dans l'Aude (Vaquer, 1990, 1991) nous paraissent former une seule et même chose.

La distinction entre les phases D1 et D2 de Provence semble perceptible en Languedoc oriental avec les variantes $5 \mathrm{a}$ et $5 \mathrm{~b}$. C. Lepère a bien montré qu'en Provence, « l'étape D semble initier une modification des tropismes régionaux marquée par des relations plus fortes [avec] les sphères NMB, Cortaillod et Lagozza [au détriment des] liens avec le Languedoc » (Lepère, 2012, p. 534) ; cependant il nous semble que cela concerne moins la Provence rhodanienne que la Provence centrale, au moins dans la phase D1. En effet, les relations entre le style 5a dont les sites sont proches du Rhône, et les séries vauclusiennes et drômoises de la phase D1 sont fortes. Elles montrent qu'un axe restreint de relations privilégiées s'établit à proximité du fleuve. Quelques éléments d'influence provençale qui s'infiltrent dans la variante $5 b$, notamment à Raffègues, rappellent la touche NMB/ Cortaillod/Lagozza (boutons en couronne contre le bord, boutons simples ou doubles sur les segmentations, cols à sillon). Cependant le développement des décors exubérants dans cette variante $5 \mathrm{~b}$ évoque à l'opposé le groupe de Bize. Considérant ces relations entre l'Aude et le Languedoc oriental, il est étonnant de constater le manque de relais géographique entre ces deux régions. S'agit-il d'une réalité ou d'un état de la recherche ?

Ces derniers styles creusent en tout cas les différences géographiques. Au moins quatre zones se démarquent : le Quercy et le Garonnais dont le style le plus récent s'étend jusque dans le Bassin de l'Aude (Cavanac) et qui montrent des contacts avec les régions atlantiques, les Pyrénées et l'Auvergne ; le Languedoc central où la documentation sur ces phases demeure très inégale ; les rives du Rhône qui montrent une forte cohésion dans le style 5a/étape D1; la Provence centrale où s'affichent le plus nettement des influences extra-culturelles.

\section{LA FIN DU NÉOLITHIQUE MOYEN}

La fin du Néolithique moyen (3650-3500 av. J.-C.) est marquée par des séries réparties, pour l'essentiel, en Provence et dans la Drôme (groupe d'Allan). C'est dans ce cadre que s'inscrit La Grange des Merveilles. L'évolution perceptible à Château Blanc (Hasler et al., 1998) concerne probablement bon nombre des autres ensembles. Il y a une gradation dans le détachement par rapport aux traditions chasséennes, mais d'une manière globale, en dehors des carènes qui ne disparaissent pas complètement au début du Néolithique final, peu d'éléments chasséens sont encore présents. Non seulement les formes segmentées évoluent vers des pots à segmentation haute, mais la perte quasi totale des éléments de préhension multiforés et des décors linéaires en creux, la simplification amorcée des étapes de finition des vases (Lepère, 2012) sortent ces ensembles du giron chasséen. Cependant, il s'agit encore de céramique lisse, ce qui pour nous est un gage d'appartenance au Néolithique moyen II. Les industries lithiques taillées des Merveilles montrent l'abandon des réseaux de diffusion du silex blond bédoulien mais préfigurent ceux des grandes lames exogènes qui caractérisent le Néolithique final (Merveilles, notamment). Si nous devons nommer ces manifestations, nous choisirions les termes de «tardi-Chasséen » à la suite d'A. Beeching qui les situe par contre au début du Néolithique final I contrairement à nous (Beeching, 2002) ou de « post-Chasséen ». La répartition géographique de ces sites est liée à la vallée du Rhône et à la plaine littorale provençale proche. Il est pour l'instant difficile de savoir s'il s'agit d'un défaut de documentation ailleurs ou d'un phénomène de faciès.

\section{CONCLUSION}

La sériation que nous avons proposée pour le Languedoc oriental mérite d'être retravaillée en prenant en compte de plus nombreux critères typologiques et en associant d'autres sites que nous n'avons pu inventorier exhaustivement jusqu'ici. En l'état, elle demeure fragile, notamment pour les styles 1 et 4 qui ne sont représentés que par un nombre restreints d'ensembles mal documentés. Une approche technologique à grande échelle sera également nécessaire à l'avenir, pour doubler les observations typologiques par l'analyse de l'évolution des chaînes opératoires des modes de façonnage.

Cependant, ce travail contribue au moins à faire connaître 
des données inédites, complète l'état des recherche sur les périodisations maintenant disponibles dans tout le Midi et apporte sa pierre à la définition du Chasséen méridional. Il reste à mener un travail collectif de réflexion sur la synchronisation des sériations méridonales et d'homogénéisation des dénominations pour permettre de poursuivre les recherches sur des bases communes. Plus largement, la question de la culture - ou du complexe - chasséen mériterait également un nouveau bilan. Le colloque «Identité du Chasséen » paraît loin (Beeching et al., 1991) et pourtant, malgré le renouvellement de la réflexion suscité à cette occasion, il semble bien difficile encore aujourd'hui, 20 ans plus tard, de bien cerner sa complexité. De nombreux points cruciaux restent à creuser concernant ses origines, sa définition, l'articulation des mécanismes d'acquisition, d'innovation et d'inertie, les modalités et les voies de diffusion de ses caractères dans d'autres cultures, la part des apports externes dans son évolution.

\section{BIBLIOGRAPHIE}

$\begin{array}{ll}\text { ADALR } & \text { Association pour le Développement de l'Archéologie en Languedoc-Roussillon } \\ \text { AEP } & \text { Archives d'écologie préhistorique } \\ \text { APRAIdF } & \text { Association pour la Promotion de la Recherche archéologique en Ile-de-France } \\ \text { ARAIdF } & \text { Association pour la Recherche archéologique en Ile-de-France } \\ \text { BMAPM } & \text { Bulletin du Musée d'anthropologie préhistorique de Monaco } \\ \text { BSASO } & \text { Bulletin de la Société d'anthropologie du Sud-Ouest } \\ \text { BSESNNG } & \text { Bulletin de la Société d'étude des sciences naturelles de Nîmes et du Gard } \\ \text { BSPF } & \text { Bulletin de la Société préhistorique française } \\ \text { CASR } & \text { Centre d'Anthropologie des Sociétés rurales } \\ \text { CLPA } & \text { Cahiers ligures de préhistoire et d'archéologie } \\ \text { CRAHL } & \text { Centre de Recherches archéologiques du Haut-Languedoc } \\ \text { JMA } & \text { Journal of Mediterranean Archaeology } \\ P S O & \text { Préhistoire du Sud-Ouest } \\ \text { RAE } & \text { Revue archéologique de l'Est } \\ \text { SPF } & \text { Société préhistorique Française } \\ \text { UISPP } & \text { Union internationale des Sciences préhistoriques et protohistoriques }\end{array}$

\section{Abelanet J.}

1970: « Une tombe néolithique : L' Arca de Calahons (Catlar, Pyrénéesorientales) », in Guilaine J., SACCHI D. (Dir.), Les Civilisations néolithiques du midi de la France, actes du colloque de Narbonne, 1970, Carcassonne, Laboratoire de Préhistoire et de Palethnologie (coll. Atacina, 5), p. 37.

Ambert P, Genna A., Taffanel O.

1988: « Contribution à l'étude du Chasséen du Minervois ", in Le Chasséen en Languedoc oriental, hommage à Jean Arnal, actes des journées d'études de Montpellier, oct. 1985, Montpellier, Montpellier, Publication de la recherche, Université Paul-Valéry, p. 25-36.

Amiel C., Jédikian G.

2003: «Aspect de la céramique du Chasséen ancien en Languedoc occidental : l'exemple du site d'Encombres à Quarante (Hérault) ", in GAScò J., Gutherz X., De Labriffe P.-A. (Dir.), Temps et espaces culturels du $6^{e}$ au $2^{e}$ millénaire en France du Sud, actes des $4^{e}$ Rencontres Méridionales de Préhistoire Récente, Nîmes, oct. 2000. Lattes, éd. de l'ADALR, Monographies d'archéologie méditerranéenne, 15, p. 389-392.

Arnal J.

1956: " La grotte de la Madeleine », Zephyrus, 7, p. 33-79.

Arnal G.-B.

1983: La grotte IV de Saint-Pierre-dela-Fage (Hérault) et le Néolithique ancien du Languedoc, Lodève, Centre de recherches archéologiques du Haut Languedoc (mémoire $\mathrm{n}^{\circ}$ 3), $195 \mathrm{p}$.

Arnal G.-B., Arnal N., Clopès J.

1991: Teyran (Hérault). Première agglomé- 
ration préhistorique à structures en pierres sèches. Approche chronologique du Chasséen méridional, Lodève, CRAHL, Mémoire nº VI, 94 p.

\section{Barral L.}

1960: «La grotte de la Madeleine (Hérault)», $B M A P M, 7$, p. 5-73.

\section{Bazile F., Lemerle P., Pellé R.}

2002: "Le Néolithique moyen de Jarnègues, Jonquières-Saint-Vincent (Gard) ", in Archéologie du TGV Méditerranée, fiches de synthèse, 1 : La Préhistoire, Lattes, éd. UMR154 du CNRS, Monographies d'Archéologie Méridionale, 8, p. 319-332.

Beeching A.

1995: « Nouveau regard sur le Néolithique ancien et moyen du bassin rhodanien », in Voruz J.-L. (Dir.), De 6000 à 2000 avant notre ère dans le Bassin rhodanien, actes des XI Rencontres sur le Néolithique de la région Rhône-Alpes, Ambérieu-en-Bugey, 1992, Ambérieu-en-Bugey, éd. SPR, Documents du Département d'Anthropologie de l'Université de Genève, 20, p. 93- 111

2002: «La fin du Chasséen et le Néolithique final dans le bassin du Rhône moyen », in Ferrari A., Visentini P. (Dir.), Il declino del mondo neolitico. Ricerche in Italia centro-settentrionale fra aspetti peninsulari, occidentali et nord-alpini, actes des Rencontres de Pordenone, 2001, éd. Museo archeologico del Friuli occidentale, quaderno, 4 , p. 67-83.

Beeching A., Binder D., Blanchet J.-C. Constantin C., Dubouloz J., Martinez R., Mordant D., Thévenot J.-P., VAQUER J. (Dir.)

1991 : Identité du Chasséen, actes du colloque international de Nemours, mai 1989, Nemours, APRAIdF, Mémoires du musée de préhistoire d'Ile-de-France, 4, $428 \mathrm{p}$.

Binder D.

1990: « Néolithique moyen et supérieur dans l'aire liguro-provençale : le cas de Giribaldi (Nice, AlpesMaritimes, France) », in Guilaine J., Gutherz X. (Dir.), Autour de Jean Arnal, Montpellier, éd. Premières Communautés Paysannes, p. 147-161.

1991: «Facteurs de variabilité des outillages lithiques chasséens dans le Sud-Est de la France ", in Beeching A., Binder D., Blanchet J.-C., Constantin C., Dubouloz J., Martinez R., Mordant D. Thévenot J.-P., VAquer J. (Dir.), Identité du Chasséen, actes du colloque international de Nemours, 1989, Nemours, éd. APRAIdF, Mémoires du musée de Préhistoire d'Île-de-France, 4, p. 261-272.

1998: « Silex blond et complexité des assem- blages lithiques dans le Néolithique liguro-provençal », in D'AnNA A., BINDER D. (Dir.), Production et identité culturelle, actes des II Rencontres méridionales de Préhistoire récente, Arles, nov. 1996, Antibes, éd. A.P.D.C.A., p. 111-128.

BINDER D.

2004: Un chantier archéologique à la loupe : villa Giribaldi (Nice), Nice, Musée archéologique Nice-Cimiez, 92p.

Binder D., Lepere C., Maggi R.

2008: "Épipaléolithique et Néolithique dans l'arc liguro-provençal : bilan et perspectives de recherche ", BMAPM, suppl. 1, p. 49-62.

Bordreuil M., Roger J.-M.

1992: «Inventaire des gisements chasséens du département du Gard », BSESNNG, 59, p. 100-126.

BRIOIS F.

1998: "Saint-Pargoire - Mas d'Embec ", AdlFI, Archéologie de la France Informations [en ligne], notice $\mathrm{n}^{\circ}$ 2004-LA-0221.

Briois F., Lea V.

2003: "Productions lithiques autochtones et identité du Chasséen : l'exemple de deux sites de la vallée du Lez (Hérault) ", in Gasco J., Gutherz X., DE LABriffe P.-A. (Dir.), Temps et espaces culturels du $\sigma^{e}$ au $2^{e}$ millénaire en France du Sud, actes IV Rencontres méridionales de Préhistoire récente, Nîmes, oct. 2000, Lattes, ADAL, Monographies d'Archéologie Méditerranéenne, 15, p. 135-142.

Buisson-Catil J.

1996: "Crillon-le-Brave - La Blaoute », Bilan scientifique de la région Provence - Alpes - Côte d'Azur, 1995, DRAC, Ministère de la Culture, p. 280-283.

Chapon P., Hasler A., Renault S., Villemeur I.

2002: « Le site chasséen de L'Héritière II à Vernègues ", in Archéologie du TGV Méditerranée, fiches de synthèse, $t$. 1 , La Préhistoire, Lattes, éd. UMR154 du CNRS, Monographies d'Archéologie Méridionale, 8, p. 203-212.

Coye N.

2007: «Les niveaux stratifiés du Néolithique moyen ", in Daveau I. (Dir.), Port Ariane (Lattes, Hérault). Construction deltaïque et utilisation d'une zone humide lors des six derniers millénaires, Lattes, éd. de l'ADALR, Lattara, 20, p. 301-330.

Crepaldi F.

2004: I rapporti tra la Cultura VBQ e la Cultura chasseana nella regione transalpina : l'analisi della produzioni ceramiche, thesè de doctorat, Université d'Udine, 2 vol.

DANGEL L.

1997: Contribution à l'étude de la céramique $d u$ Néolithique ancien et du Néolithique moyen : l'abri de la Font des Pigeons à Châteauneuf-lès-Martigues, fouille Jean Courtin 1979, mémoire de Maîtrise, Aix-en-Provence, Université de Provence, 2 vol.

Davaux I. (Dir.)

2007: Port Ariane (Lattes, Hérault). Construction deltaïque et utilisation d'une zone humide lors des six derniers millénaires, Lattes, éd. de l'ADALR, Lattara, 20, 634 p.

Escalon De Fonton M., Onoratini G.

1991: « Découverte d'un Néolithique moyen dans la grotte de l'Adaouste à Jouques (Bouches-du-Rhône) une nouvelle phase évolutive du Néolithique moyen, ante-chasséenne », BSPF Bulletin, 88, 5, p. 138-140.

Fenouillet M., Vaquer J.

1974: « La fosse néolithique du Lavous, Grand-Gallargues (Gard) », $B S P F, 71,8-9$, p. 231-235.

Fouéré P., Marsac P., Ranché C.

2003: "Une occupation Chalcolithique et Chasséen ancien au Pla de Peyre, Creissels (Aveyron) ", in Gascò J., Gutherz X., De Labriffe P.-A. (Dir.), Temps et espaces culturels $d u \sigma^{e}$ au $2^{e}$ millénaire en France du Sud, actes des $4^{e}$ Rencontres Méridionales de Préhistoire Récente, Nîmes, oct. 2000, Lattes, éd. de l'ADALR, Monographies d'Archéologie Méditerranéenne, 15, p. 32.

Francois P.

2002: Les productions céramiques $d u$ Chasséen de Villeneuve-Tolosane, évolution stylistique et comparaisons avec les autres faciès chasséens d'Europe occidentale, thèse $\mathrm{de}$ Doctorat, Toulouse, EHESS, 2 vol.

2007: Les styles céramiques du Chasséen de Villeneuve-Tolosane : évolution et comparaisons, Oxford, Archeopress, BAR, International Series, 1711, 189 p.

Furestier R., Sendra B., Gourlin B., Cockin G., Gourichon L., Howarth L., Lea V., Legrand A., Lepère C., Michel J., Rousselet O., Servelle C.

2012: «Évolution du Chasséen montpelliérain : premiers résultats des fouilles préventives du site de la ZAC SaintAntoine à Saint-Aunès (Hérault) », in Perrin T., Sénépart I., Cauliez J., Bonnardin S., Thirault E. (Dir.), Dynamismes et rythmes évolutifs des sociétés de la Préhistoire Récente, 
actes des $g^{e}$ Rencontres méridionales de Préhistoire récente, SaintGeorges-de-Didonne/Royan, oct. 2010, Toulouse, éd. AEP.

Gaillard A.

2003 : « Le site d'Auriac-Golf à Carcassonne (Aude) : une aire d'ensilage du Chasséen ancien ", in GASCÒ J., Gutherz X., De Labriffe P.-A. (Dir.), Temps et espaces culturels du $\sigma^{e}$ au $2^{e}$ millénaire en France du Sud, actes des $4^{e}$ Rencontres Méridionales de Préhistoire Récente, Nîmes, oct. 2000, Lattes, éd. de l'ADALR, Monographies d'Archéologie Méditerranéenne, 15, p. 393-396.

GaLin W.

2012: Diversité et variabilité des industries lithiques du Chasséen méridional : le cas de Saint-Michel du Touch (Toulouse, Haute-Garonne), mémoire de Master 2, Toulouse, Université de Toulouse le Mirail, 2 vol.

Gandelin M.

2011: Les enceintes chasséennes de Villeneuve-Tolosane et de Cugnaux dans leur contexte du Néolithique moyen européen, Toulouse, éd. AEP, 506 p

Garrido M.-C.

1990: Inventaire des sites néolithiques et chalcolithiques dans le lunellois, mémoire de Maîtrise, Montpellier, Université Paul-Valéry, 214 p.

GeorJon C.

2000: «La Roquette (Tresques, Gard) et le Néolithique final du bassin bas-rhodanien », Gallia Préhistoire, 41, p. 1-45.

2002: "Roquemaure - La Ramière, le gisement néolithique $\mathrm{du}$ Roc de Peillet ", in Archéologie du TGV Méditerranée, fiches de synthèse, $t$. 1, La Préhistoire, Lattes, éd. UMR 154 du CNRS, Monographies d'Archéologie Méridionale, 8, p. 247-254.

2003: «Chronologie, variabilité et phénomènes de récurrence dans la céramique chasséenne de la basse vallée du Lez (Hérault) », in Gascò J., Gutherz X., De Labriffe P.-A. (Dir.), Temps et espaces culturels du $\sigma^{e}$ au $2^{e}$ millénaire en France du Sud, actes des 4 e Rencontres Méridionales de Préhistoire Récente, Nîmes, oct. 2000, Lattes, éd. de l'ADALR, Monographies d'Archéologie Méditerranéenne, 15, p. 115-134.

2007: «La céramique chasséenne de Port Ariane III ", in Daveau I. (Dir.), Port Ariane (Lattes, Hérault). Construction deltaïque et utilisation d'une zone humide lors des six derniers millénaires, Lattes, éd. de l'ADALR, Lattara, 20, p. 331-342.

Gernigon K.

2004: Productions matérielles et Identités culturelles dans le Néolithique d'Europe occidentale : réflexions autour de la céramique chasséenne en Quercy, thèse de Doctorat, Toulouse, Université de Toulouse-le Mirail, 3 vol.

\section{Guilaine J.}

1959: «Les sepultures en fosse de la Laiga (Cournanel, Aude) », BSPF, 56, 11-12, p. 681-684.

1962: «Sépultures néolithiques dans le sud de la France », Zephyrus, 13, p. 17-29.

1997a: « Cerny et le Sud », in Constantin C., Mordant D., Simonin D. (Dir.), La culture de Cerny, nouvelle économie, nouvelle société au Néolithique, actes du Colloque international de Nemours 1994, Nemours, ARAIdF, Mémoires du Musée de Préhistoire d'Ile-deFrance, 6, p. 631-644.

1997b: « La question bizienne. Historique », in Guilaine J., Barthès P. (Dir.), La Poste Vieille. De l'enceinte néolithique à la bastide d'Alzau, Toulouse, Centre d'Anthropologie, Carcassonne, Archéologie en terre d'Aude, p. 168-193.

Guilaine J., Amiel C., Barthès P., Coularou J., Vaquer J.

1990: «Le Chasséen de l'abri de FontJuvénal », in Guilaine J., Gutherz X. (Dir.), Autour de Jean Arnal, Montpellier, éd. de l'Association Recherches sur les premières communautés paysannes en Méditerranée occidentales, p. 163-175.

Guilaine J., Vaquer J.

1973: « Le site chasséen d'Auriac, commune de Carcassonne (Aude) », BSPF, 70, études et travaux, p. 367-384.

Hasler A., Chevillot P., Collet H., Durand C., Renault S., Richier A.

1998: « La nécropole tumulaire néolithique de Château Blanc (Ventabren, Bouches-du-Rhône) », in D'AnNA A., Binder D. (Dir.), Production et identité culturelle, actes des $2^{e}$ Rencontres méridionales de Préhistoire récente, Arles, nov. 1996, Antibes, éd. APDCA, p. 403-414.

Houdré J.-J., Vital J.

1979: « Le gisement chasséen ancien du Pirou (commune de Polignac, HauteLoire) », $B S P F, 76,10-12$, p. 355-378.

\section{Jallet F., Georjon C.}

sous presse: «Entre Sud et Nord, l'Auvergne au $\mathrm{V}^{\mathrm{e}}$ millénaire : préliminaires à une synthèse culturelle », in Transitions, ruptures et continuités en Préhistoire, XXVII Congrès préhistorique de France, session I : 4500-4200 avant notre ère : le début des inégalités?, Bordeaux - Les Eyzies-de-Tayac, mai-juin 2010
Jallot L., Georjon C., LÉa V., Wattez J. 2000: «Principaux résultats de l'étude du site chasséen ancien de Jacques Coeur II (Port Marianne, Montpellier, Hérault) », in Leduc M., VALdEYRON N., Vaquer J. (Dir.), Sociétés et espaces, actes des $3^{e}$ Rencontres Méridionales de Préhistoire Récente, Toulouse, nov. 1998, Toulouse, éd. AEP, p. 281-303.

JÉDIKIAN G.

1999: Étude de la céramique du puits chasséen de Villeneuve-Tolosane, mémoire de DEA, Toulouse, EHESS, 96 p.

2000: «Statistique et typologie de la céramique chasséenne, l'exemple d'Auriac à Carcassonne, Aude "), in Leduc M., VAldeYron N., VAQuer J. (Dir.), Sociétés et espaces, actes des $3^{e}$ Rencontres Méridionales de Préhistoire Récente, Toulouse, nov. 1998, Toulouse, éd. AEP, p. 305-312.

LEA V.

2003: «Un atelier de fabrication de microperçoirs au Chasséen : le site de la Cabre (Var) », BSPF, 100, 3, p. 517-532.

2004a : Les industries lithiques du Chasséen en Languedoc oriental : caractérisation par l'analyse technologique, Oxford, éd. BAR International Series, $1232,215 \mathrm{p}$

2004b: «Les productions sur galets locaux du Néolithique moyen du sud de la France», in Dartevelle H. (Dir.), Auvergne et Midi, $V^{e}$ Rencontres méridionales de Préhistoire récente, Clermont-Ferrand, nov. 2002, éd. PSO, suppl. n 9, p. 395-404.

2004c: «Centres de production et diffusion des silex bédouliens au Chasséen», Gallia Préhistoire, 46, p. 231-250.

2012: "The Diffusion of Obsidian in the North-West Mediterranean: Toward a New Model of the Chassey culture? », $J M A, 25,2$, p. 147-173.

Léa V., Georjon C., Lepère C., Sénépart I. 2004a: «Chasséen vauclusien qui es-tu ?», in Buisson-Catil J., Guilcher A., Hussy C., Olive M., Pagni M. (Dir.), Vaucluse préhistorique, Le Pontet, éd. A. Barthélémy, p. 163-200.

Lea V., Gassin B., Briois F.

2004b: «Fonctionnement des réseaux de diffusion des silex bédouliens du V au IV millénaire : questions ouvertes », in Dartevelle H. (Dir.), Auvergne et Midi, Ve Rencontres méridionales de Préhistoire récente, ClermontFerrand, nov. 2002, PSO, suppl. n ${ }^{\circ}$, p. $405-420$.

Léa V., Binder D., Briois F., Vaquer J.

2007: «Le Chasséen méridional à lamelles d'Arnal : évolution de notre perception des industries lithiques », in Evin J. (Dir.), Un siècle de construction 
$d u$ discours scientifique en Préhistoire, vol. 3, Paris, SPF, CNRS, p. 263-275.

Lea V., Gassin B., Linton J.

2009: «Quelles armatures de projectiles pour le midi méditerranéen et ses marges du milieu $\mathrm{du} \mathrm{V}^{\mathrm{e}}$ au milieu du $\mathrm{IV}^{\mathrm{e}}$ millénaire ? », Gallia Préhistoire, 51, p. $155-177$

Léa V., Pélissier M., Cratuze B., Boucetta S., Lepère C.

2010: «Renouvellement des données sur la diffusion de l'obsidienne sarde en contexte chasséen (Midi de la France) : la découverte du site des Terres Longues (Trets, Bouches-duRhône) ", in L'ossidiana del Monte Arci nel Mediterraneo, nuovi apporti sulla diffusione, sui sistemi di produzione e sulla loro cronologia, actes $d u 5^{e}$ Congrès international, Pau, juin 2008, Ales, ed. Nur, p. 157-185.

Lemercier O.

2002: "Les occupations néolithiques de Mondragon - Les Juilléras (Vaucluse) », in Archéologie du TGV Méditerranée, fiches de synthèse, t. 1, la Préhistoire, Lattes, publications de l'UMR154 du CNRS, Monographies d'archéologie méditerranéenne, 8, p. 147-172.

LEPÈre C.

2002: La céramique pré-chasséenne d'Escanin, mémoire de Maîtrise, Aixen-Provence, Université de Provence, Aix-Marseille I, $168 \mathrm{p}$.

2003: Approche comparative des ensembles céramiques du Néolithique supérieur de Pertus II (Alpes-de-Haute-Provence) et Escanin II (Bouches-du-Rhône), mémoire de DEA, Aix-en-Provence, Université d'Aix-Marseille I, 2 vol.

2009: Identités et transferts culturels dans le domaine circumalpin : l'exemple des productions céramiques du Chasséen provençal, thèse de Doctorat, Université Aix-Marseille I, Aix-enProvence, 2 vol

2012: «Chronologie des productions céramiques et dynamique culturelle du Chasséen en Provence », BSPF, 109, 3, p. 513-546.

LOISON G., GISCLON J.-L.

1991 : « La nécropole de Pontcharaud 2 dans le cadre de nouvelles approches du peuplement néolithique de la BasseAuvergne », in BeEching A., Binder D., Blanchet J.-C., Constantin C., Dubouloz J., Martinez R., Mordant D., ThÉvenot J.-P., VAQuer J. (Dir.), Identité du Chasséen, actes du colloque international de Nemours, mai 1989, Nemours, APRAIdF, Mémoires du musée de préhistoire d'Ile-de-France, 4, p. 399-408.
Louis M.

1953: «Le Néolithique dans les Baumes Latrone », CLPA, 2, p. 49-138.

Luzi C., Courtin J.

2001: « La céramique des niveaux préchasséens de la Baume Fontbrégoua (Salernes, Var) », BSPF, 98, p. 471-484.

Marchand G., Vaquer J.

1976: « Le gisement chasséen de l'Hournède à Saint-Nazaire-de-Pézan (Hérault) ", Études préhistoriques, 13, p. 19-22.

\section{Mendoza A., Prades H.}

1988: " Le gisement chasséen de Lattes ", in Le Chasséen en Languedoc oriental. Hommage à Jean Arnal, actes des journées d'étude de Montpellier, oct. 1985, Montpellier, Université Paul-Valéry (Préhistoire U. P. V. nº 1), p. 83-95.

Monnet C., Bazile F., Georjon C., Di Pietro-Sirven R.

2002: « La Grange des Merveilles I : un habitat du Néolithique récent ", in Archéologie du TGV Méditerranée, fiches de synthèse, t. 1, La Préhistoire, Lattes, éd. UMR154 du CNRS, Monographies d'Archéologie Méridionale, 8, p. 275-285.

\section{Montuardin R., Rouquette D.}

1988: " Approche statistique de la céramique chasséenne de Raffègues/ Mas de Guarric. Zone industrielle de Mèze (Hérault) », in Le Chasséen en Languedoc oriental. Hommage à Jean Arnal, actes des journées d'étude de Montpellier, oct. 1985, Montpellier, Université Paul-Valéry (Préhistoire U.P.V. $\left.n^{\circ} 1\right)$, p. 153-164.

NICOD P.-Y.

2009: « Les céramiques du Néolithique moyen I (couches 52 à 48) », in VorUZ J.-L. (Dir.), La grotte du Gardon (Ain), vol. 1 : le site et la séquence néolithique des couches 60 à 47 , Toulouse, éd. Centre de recherche sur la Préhistoire et la Protohistoire de la Méditerranée, éd. AEP, p. 501-536.

Noret C.

2002: «L'occupation chasséenne du site du Réal à Montfrin (Gard) ", in Archéologie du TGV Méditerranée, fiches de synthèse, t. 1, La Préhistoire, Lattes, éd. UMR154 du CNRS, Monographies d'Achéologie méditerranéenne, 8, p. 287-298.

Onoratini G., Mafart B., Joris C., BARONI I.

1997: «Les occupations humaines de la grotte de l'Adaouste (Jouques, Bouchesdu-Rhône) », Quaternaire, 8, 2-3, p. 175-187.

Reimer P. J., Baillie M. G. L., BARD E, $E T A L$.

2009: « Intercal09 and Marine09
Radiocarbon age calibration curves, 0-50000 years cal BP ", Radiocarbon, 51, 4, p. 1111-1150.

Rodriguez G.

1983: La grotte de Camprafaud, Saint-Ponsde-Thomières, éd. Fédération archéologique de l'Hérault, groupe archéologique Saintponais, $417 \mathrm{p}$.

Roger J.-M., Valette P.

1988: «La céramique chasséenne du Puech de la Fontaine (Congénies, Gard) », in Le Chasséen en Languedoc oriental. Hommage à Jean Arnal, actes des Journées d'études, Montpellier, oct. 1985, Montpellier, Université Paul-Valéry (Préhistoire U.P.V. n ${ }^{\circ} 1$ ), p. 173-192.

Sargiano J.-P., Van Willigen S., D'Anna A., Renault S., Hunger K., Woerle-Soarès M., Gaday R.

2010: « Les Bagnoles à l'Isle-sur-la-Sorgue (Vaucluse). Aspects nouveaux dans le Néolithique moyen du midi de la France ", Gallia Préhistoire, 52, p. 193-239.

Servelle C.

1980: « Deux vases chasséens exceptionnels des Monges (Launaguet, Haute-Garonne) ", BSPF, 77, 10-12, p. 385-396.

Simonnet G.

1976: « Le village chasséen de Saint Michel du Touch à Toulouse (HauteGaronne) », in Clottes J. (Dir.), $I X^{e}$ Congrès de l'Union internationale des sciences préhistoriques et protohistoriques, Livret-guide de l'excursion A5: Pyrénées, Nice, UNPP, p. $15-34$

ThÉVEnot J.-P.

2005: Le Camp de Chassey, Chassey-leCamp, Saône-et-Loire, Les niveaux néolithiques du rempart de "la Redoute ", Dijon, éd. RAE, $22^{\mathrm{e}}$ supplément, $464 \mathrm{p}$.

Timsit D.

1994: Étude techno-morphologique de la céramique néolithique et chalcolithique méridionale de la collection P. Raymond (musée des antiquités nationales de Saint-Germain-en-Laye), mémoire de Maîtrise, Paris, Université de Paris I, 2 vol.

TIXIER J.

1979: « Expérience de taille de flèches à tranchant transversal du type de l'abri Jean-Cros », in Guilaine J. (Dir.), L'Abri Jean-Cros, Toulouse, éd. CAR, p. $127-130$

VAN Villigen S.

2010: « La stratigraphie du Mourre de la 
Barque (Jouques, Bouches-du-Rhône) et la fin du Néolithique moyen en Provence occidentale, in Lemercier O., Furestier R., Blaise E., $4^{e}$ millénaire. La transition du Néolithique moyen au Néolithique final dans le sud-est de la France et les régions voisines, Lattes, publications de l'UMR154 du CNRS, Monographies d'archéologie méditerranéenne, 27, p. 45-74.

Van Willigen S., D'Anna A., Renault S. SARgIano J.-P.

2012: « Le sud-est de la France entre 4400 et 3400 avant notre ère. Sériation céramique et outillage lithique ", Préhistoires Méditerranéennes [en ligne], 2, 2011.

VAQUer J.

1975: La céramique chasséenne du Languedoc, Carcassonne, Laboratoire de Préhistoire et de Palethnologie, 368 p. 1989: «Innovation et inertie dans le processus de néolithisation en Languedoc occidental ", in Aurenche O., Cauvin J. (Dir.), Néolithisations, Oxford, BAR, Interantional Series 516, p. $187-198$.

1990: «L'évolution du Chasséen méridional, essai dans le bassin de l'Aude ", in Guilaine J., Gutherz X. (Dir.), Autour de Jean Arnal, Montpellier, éd. de l'association Recherches sur les premières communautés paysannes en Méditerranée occidentales, p. 177-189.

1991: « Aspects du Chasséen en Languedoc occidental. Habitat et culture matérielle ", in BeEching A., Binder D., Blanchet J.-C., Constantin C., Dubouloz J., Martinez R., Mordant D., Thévenot J.-P., Vaquer J. (Dir.), Identité du Chasséen, actes du colloque international de Nemours, mai 1989.
Nemours, APRAIdF, Mémoires du musée de préhistoire d'Ile-de-France, 4, p. 27-37.

1998: " Le Midi méditerranéen de la France ", in Guilaine J. (Dir.), Atlas du Néolithique européen. Vol. $2 A$. L'Europe occidentale, Liège, éd. de l'UISPP, ERAUL, 46, p. 413-500.

VAQUer J., JÉdikian G.

2003: « La Salle, Carcassonne (Aude). Un habitat de plein air du groupe de Bize », $B S P F, 100,2$, p. 323-351.

Vernet G., Barthélémy S.

1983: « Nouvelles données sur le Chasséen en Basse-Auvergne ", BSASO, 18, 3, 1983 , p. $127-132$

Vignaud A.

1993: « Le Boulou, Cortal d'En Kircq », Gallia informations, 1, p. 163. 